\title{
SOLVING THE EINSTEIN EQUATIONS
}

BY

\author{
LIPSCHITZ CONTINUOUS METRICS: \\ SHOCK WAVES IN GENERAL RELATIVITY
}

Jeff Groah ${ }^{1}$ Joel Smoller ${ }^{2}$ Blake Temple

January 1, 2001

\section{Introduction}

These notes address mathematical issues that arise when one attempts to incorporate shock waves into Einstein's theory of General Relativity. At the start, one is led to consider solutions of the Einstein equations when the spacetime metric is only Lipschitz continuous, and this is the main topic of this article. In Section 1, (which is taken from [36]), we introduce General Relativity and the Einstein equations, and then we begin the discussion in Subsection 2.2, (taken from [9]), by writing down the Einstein equations for a perfect fluid assuming spherical symmetry, and assuming standard Schwarzschild coordinates. We point out that the equations imply that the metric components of solutions can be at best Lipschitz continuous functions of the coordinates when shock waves are present, (that is, the metric components, viewed as functions of the spacetime coordinates, lie at best within the class $C^{0,1}$ of functions that are continuous with Holder exponent 1, [6]). We then write down a system of conservation laws with source terms that is weakly equivalent to these equations, and this helps explain the less than expected regularity of the metric. A rigorous derivation of the equivalence of these equations is the topic of Section 5. Now the Lipschitz continuity of

\footnotetext{
${ }^{1}$ Department of Mathematics, University of California, Davis, Davis, CA 95616

${ }^{2}$ Department of Mathematics, University of Michigan, Ann Arbor, MI 48109; Supported in part by NSF Applied Mathematics Grant Number DMS-89-05205 and the Institute of Theoretical Fynamics, UC-Davis.

${ }^{3}$ Department of Mathematics, University of California, Davis, Davis CA 95616; Supported in part by NSF Applied Mathematics Grant Number DMS-86-13450 and by the Institute of Theoretical Dynamics, UC-Davis.
} 
the metric components is interesting because the curvature tensor, a quantity determined by second derivatives of the metric tensor, must remain free of delta function sources in order to be a bona fida weak solution of the equations. This motivates the discussion in Section 2, (taken from [29]), which presents the general theory of matching spacetime metrics Lipschitz continuously across smooth shock surfaces. In Section 3, (which is taken from $[31,36])$, we develop a theory for matching a Friedmann-RobertsonWalker metric of cosmology, to a Tolman-Oppenheimer-Volkoff metric for a static fluid sphere, across a shock wave interface, and in Section 4, (taken from [30]), we use this theory to derive a class of exact shock wave solutions of the Einstein equations that model blast waves in General Relativity. In these exact solutions, the Big Bang singularity of the FRW metric is replaced by a shock wave explosion, and the outgoing shock wave lies at the leading edge of what is interpreted as the expansion of the galaxies in the cosmological interpretation of the FRW metric. The construction of these exact solutions takes advantage of being able to work with metrics in the lower smoothness class of $C^{0,1}$. In Section 5, (taken from [9]), we show that the spherically symmetric Einstein equations written in standard Schwarzschild coordinates, (that is, the equations which began the discussion in Section 1), are weakly equivalent to a system of conservation laws with source terms. This reformulation of the equations shows that we can expect solutions with shock waves to exist, and helps explain the Lipschitz regularity of the metric components when shocks are present. The system of equations derived in Section 5 is also the starting point for the existence theory given in [10]. The main theorem in [10] is stated in Subsection 2.2. This result confirms what is indicated by the equations derived in Section 5, and demonstrates rigorously that the initial value problem for the Einstein equations, (assuming perfect fluid and spherical symmetry), is consistent for initial density and velocity profiles that are discontinuous functions that are only locally of bounded total variation. Said differently, the result demonstrates that the Einstein equations of General Relativity are meaningful in the presence of arbitrary numbers of interacting shock waves, of arbitrary strength.

The class $C^{0,1}$ is one derivative less smooth than the Einstein equations suggest the metric components ought to be, and in fact, the singularity theorems in [11] presume that metrics are in the smoothness class $C^{1,1}$, one degree smoother, c.f. [11], page 284. One of the remarkable features of the results of Section 2 is that for smooth shock surfaces, there always exist co- 
ordinate transformations that smooth the components of the gravitational metric to $C^{1,1}$, and these coordinates can be taken to be the Gaussian normal coordinates of the surface. However, the Gaussian normal coordinates break down at points of interaction of shock waves, and thus it remains an open problem whether general Lipschitz continuous solutions of the Einstein equations can always be smoothed by coordinate transformation. This leads to the following interesting dichotomy: If such a coordinate transformation does not always exist, then solutions of the Einstein equations are one degree less smooth than previously assumed; and if such a transformation does exist, then it defines a mapping that takes weak solutions of the Einstein equations to strong solutions. In the latter case, it follows that the theory of distributions and the Rankine-Hugoniot jump conditions for shock waves need not be imposed as extra conditions on the relativistic compressible Euler equations in General Relativity, but rather must follow as logical consequences of the strong formulation of the Einstein equations by themselves.

\section{Spacetime and the Gravitational Metric Ten- sor}

In Einstein's theory of General Relativity, all properties of the gravitational field are determined by the gravitational metric tensor $g$, a Lorentzian metric that describes a continuous field of symmetric bilinear forms of signature $(-1,1,1,1)$, defined at each point of a four dimensional manifold $M$ called "spacetime." Freefall paths through the gravitational field are the geodesics of the metric; the non-rotating vectors carried by an observer in freefall are those vectors that are parallel transported by the (unique symmetric) connection determined by $g$; spatial lengths of objects correspond to the lengths of the spacelike curves that define their shape - length measured by the metric $g$; and time changes for an observer are determined by the length of the observer's timelike curve through spacetime, as measured by the metric $g$.

The length of a curve in spacetime is computed by integrating the element of arclength $d s$ along the curve, where, in a given coordinate system on spacetime, $d s$ is defined by 


$$
d s^{2}=g_{i j} d x^{i} d x^{j}
$$

Here we adopt the Einstein summation convention whereby repeated updown indices are assumed to be summed from 0 to 3 . A coordinate system on spacetime is a regular map that takes a neighborhood $U_{x}$ of spacetime to $\mathbf{R}^{4}$, $x: U_{x} \rightarrow \mathbf{R}^{4}$. Since spacetime is a manifold, it can be covered by coordinate charts. We let $x=\left(x^{0}, x^{1}, x^{2}, x^{3}\right)$ denote both the coordinate map and the coordinates of a point $x(P) \in \mathbf{R}^{4}$. The functions $g_{i j}(x), i, j=0,1,2,3$, are the $x$-components of the metric $g$. At each point $x$, the matrix $g_{i j}$ determines the lengths of tangent vectors in terms of their components relative to the $x$-coordinate basis $\left\{\frac{\partial}{\partial x^{i}}\right\}$. That is, in $x$-coordinates, the tangent vector to a curve $x(\xi)$, (as parameterized in $x$-coordinates), is given by $X(\xi)=\dot{x}^{i} \frac{\partial}{\partial x^{i}}$, (dot denotes $\frac{d}{d \xi}$ ), so that along the curve $x(\xi)$, the increment $d x^{i}$ in the $x^{i}$-coordinate, in the direction of the curve, is given by $d x^{i}=\dot{x}^{i} d \xi$. Thus, according to $(2.1)$, the increment in arclength along a curve $x(\xi)$ is given in terms of the increment in the parameter $\xi$ by

$$
d s^{2}=g_{i j} \dot{x}^{i} \dot{x}^{j} d \xi^{2}=\|X(\xi)\|^{2} d \xi^{2},
$$

so that, the length of an arbitrary vector $X=X^{i} \frac{\partial}{\partial x^{i}}$ is given by

$$
\|X\|^{2}=g_{i j} X^{i} X^{j}
$$

where again we assume summation over repeated up-down indices. We conclude that the length of a curve is just the integral of the $g$-length of its tangent vector along the curve. Under change of coordinates $x \rightarrow y$, a vector $X^{i} \frac{\partial}{\partial x^{i}}$ transforms to $X^{\alpha} \frac{\partial}{\partial y^{\alpha}}$ according to the tensor transformation laws

$$
X^{\alpha}=\frac{\partial y^{\alpha}}{\partial x^{i}} X^{i}, \quad \frac{\partial}{\partial y^{\alpha}}=\frac{\partial x^{i}}{\partial y^{\alpha}} \frac{\partial}{\partial x^{i}}
$$

(Our slightly ambiguous notation is that indices $i, j, k, \ldots$ label components in $x$-coordinates, and $\alpha, \beta, \gamma, \ldots$ label components in $y$-coordinates. So, for example, $X^{i}$ is the $x^{i}$-component of the tangent vector $X, X^{\alpha}$ is the $y^{\alpha}$ component of $X$, etc. This works quite well, but tensors must be re-labeled when indices are evaluated.) It follows that the metric tensor transforms according to the tensor transformation law 


$$
g_{\alpha \beta}=g_{i j} \frac{\partial x^{i}}{\partial y^{\alpha}} \frac{\partial x^{j}}{\partial y^{\beta}} .
$$

That is, at each point, $g$ transforms by the matrix transformation law

$$
\bar{g}=A^{t} g A
$$

for a bilinear form, because the matrix $A=\frac{\partial x^{j}}{\partial y^{\beta}}$ transforms the vector components of the $y$-basis $\left\{\frac{\partial}{\partial y^{\alpha}}\right\}$ over to their components relative to the $x$-basis $\left\{\frac{\partial}{\partial x^{i}}\right\}$. The Einstein summation convention keeps track of the coordinate transformation laws as in (2.2) and (2.3) so long as we keep the indices on coordinate functions "up" (as in $x^{i}$ ), coordinate basis indices "down" (as in $\frac{\partial}{\partial x^{i}}$ ), indices on vector components "up", (as in $X^{i}$ so that $X=X^{i} \frac{\partial}{\partial x^{i}}$ ), indices on basis 1-forms "up" (as in $d x^{i}$ ), and indices on components of 1forms down (as in $\omega_{i}$ so that $\omega=\omega_{i} d x^{i}$ ). In general, a tensor of type $(k, l)$ is said to have $k$-contravariant indices (up) and $l$-covariant indices (down) if the components in a given coordinate system transform according to the tensor transformation law

$$
T_{\beta_{1}, \ldots, \beta_{l}}^{\alpha_{1}, \ldots, \alpha_{k}}=T_{j_{1}, \ldots, j_{l}}^{i_{1}, \ldots, i_{k}} \frac{\partial y^{\alpha_{1}}}{\partial x^{i_{1}}} \cdots \frac{\partial y^{\alpha_{k}}}{\partial x^{i_{k}}} \frac{\partial x^{j_{1}}}{\partial y^{\beta_{1}}} \cdots \frac{\partial x^{j_{l}}}{\partial y^{\beta_{l}}}
$$

Here the (matrix) Jacobian satisfies $\frac{\partial x}{\partial y}=\left(\frac{\partial y}{\partial x}\right)^{-1}$, and by letting

$$
\left(g^{i j}\right)=\left(g_{i j}\right)^{-1},
$$

we can raise or lower an index by contracting the index with the metric; that is, for example,

$$
T_{j}^{i}=T_{\sigma j} g^{\sigma i}
$$

raises the index $i$. In the modern theory of differential geometry, $T_{j_{1}, \ldots, j_{l}}^{i_{1}, \ldots, i_{k}}$ are viewed as the components on the tensor products $\left\{\frac{\partial}{\partial x^{i_{1}}} \otimes \cdots \otimes \frac{\partial}{\partial x^{i k}} \otimes d x^{j_{1}} \otimes \cdots \otimes d x^{j_{l}}\right\}$ which form a basis for the set of operators that act linearly on $k$ copies of $T^{*} M$ and $l$ copies of $T M$, c.f. [4].

Freefall paths through a gravitational field are geodesics of the spacetime metric $g$. For example, the planets follow geodesics of the gravitational metric generated by the Sun, (approximated by the Schwarzschild metric outside 
the surface of the Sun, and by a Tolman-Oppenheimer-Volkoff (TOV) metric inside the surface of the Sun), and according to the standard theory of cosmology, the galaxies follow geodesics of a Friedmann-Robertson-Walker (FRW) metric. In spherical coordinates $x=(t, r, \theta, \phi)$, the Schwarzschild line element is given by

$$
d s^{2}=-\left(1-\frac{2 \mathcal{G} M_{0}}{r}\right) d t^{2}+\left(1-\frac{2 \mathcal{G} M_{0}}{r}\right)^{-1} d r^{2}+r^{2} d \Omega^{2},
$$

the TOV line element is given by

$$
d s^{2}=-B(r) d t^{2}+\left(1-\frac{2 \mathcal{G} M(r)}{r}\right)^{-1} d r^{2}+r^{2} d \Omega^{2},
$$

and the FRW line element is given by

$$
d s^{2}=-d t^{2}+R(t)^{2}\left(\frac{d r^{2}}{1-k r^{2}}+r^{2} d \Omega^{2}\right) .
$$

The line element determines the metric components $g_{i j}$ through the identity (2.1). Here $\mathcal{G}$ denotes Newton's gravitational constant, $M_{0}$ denotes the mass of the Sun (or a star), $M(r)$ denotes the total mass inside radius $r$, (a function that tends smoothly to $M_{0}$ at the star surface), $B(r)$ is a function that tends smoothly to $1-2 \mathcal{G} M_{0} / r$ at the star surface, $H=\frac{\dot{R}(t)}{R(t)}$ is the Hubble "constant", and $d \Omega^{2}=d \theta^{2}+\sin ^{2}(\theta) d \phi^{2}$ denotes the standard line element on the unit 2-sphere. (Here $2 \mathcal{G} M=\frac{2 \mathcal{G} M}{c^{2}}$, and we take $c=1$, [4].)

Each of the metrics (2.4)-(2.6) is a special case of a general spherically symmetric spacetime metric of the form

$$
d s^{2}=-A(r, t) d t^{2}+B(r, t) d r^{2}+2 D(r, t) d r d t+C(r, t) d \Omega^{2},
$$

where $A, B, C, D$ are arbitrary, smooth, positive functions. A spherically symmetric metric is said to be in standard Schwarzschild coordinates, (or the standard coordinate gauge), if it takes the simpler form

$$
d s^{2}=-A(r, t) d t^{2}+B(r, t) d r^{2}+r^{2} d \Omega^{2} .
$$

It is well known that, in general, there always exists a coordinate transformation that takes an arbitrary metric of form (2.7) over to the simpler 
form (2.8), [42]. In Section 2.2 below we carry out this reduction with an eye toward anticipating the regularity of the metric components $A$ and $B$, $[42,9])$.

The geodesics of a metric are paths $x(s)$ of extremal length, determined by the geodesic equation

$$
\frac{d^{2} x^{i}}{d s^{2}}=\Gamma_{j k}^{i} \frac{d x^{j}}{d s} \frac{d x^{k}}{d s}
$$

where the so called Christoffel symbols or connection coefficients $\Gamma_{j k}^{i}$ are defined by

$$
\Gamma_{j k}^{i}=\frac{1}{2} g^{\sigma i}\left\{-g_{j k, \sigma}+g_{\sigma j, k}+g_{k \sigma, j}\right\} .
$$

(Here ", $k$ " denotes the classical derivative in direction $x^{k}$.) The Christoffel symbols $\Gamma_{j k}^{i}$ are the central objects of differential geometry that do not transform like a tensor. Indeed, they fail to be tensorial by exactly the amount required to convert coordinate differentiation of vector components into a tensorial operation. That is, for a vector field $Y$, let $Y^{i}$ denote the $x^{i}$-component of $Y$. The covariant derivative $\nabla$ is defined by

$$
\nabla_{\frac{\partial}{\partial x^{\sigma}}} Y=Z
$$

where, letting semi-colon denote covariant differentiation, $Z$ defines a vector field with $x$-components

$$
Z^{i}=Y_{; \sigma}^{i} \equiv \frac{\partial Y^{i}}{\partial x^{\sigma}}-\Gamma_{\sigma k}^{i} Y^{k}
$$

For arbitrary vector fields $X$ and $Y$, one defines the covariant derivative $\nabla_{X} Y$ by

$$
\nabla_{X} Y=X^{\sigma} \nabla_{\frac{\partial}{\partial x^{\sigma}}} Y \equiv X^{\sigma} Y_{; \sigma}^{i} \frac{\partial}{\partial x^{i}} .
$$

We say that a vector field $Y$ is parallel along a curve whose tangent vector is $X$ if

$$
\nabla_{X} Y=0
$$

all along the curve. It follows that the covariant derivative $\nabla_{X} Y$ measures the rate at which the vector field $Y$ diverges from the parallel translation of 
$Y$ in the direction of $X$. In a similar fashion, one can define the covariant derivative $\nabla T$ of any $(k, l)$ tensor $T$ as the $(k, l+1)$ tensor with components

$$
T_{j_{1}, \ldots, j_{l} ; \sigma}^{i_{1}, \ldots, i_{k}}
$$

For example, for a $(1,1)$ tensor $T$,

$$
T_{j ; \sigma}^{i}=T_{j, \sigma}^{i}-\Gamma_{\tau \sigma}^{i} T_{j}^{\tau}+\Gamma_{j \sigma}^{\tau} T_{\tau}^{i} .
$$

More generally, to compute $\nabla T$ for a $(k, l)$ tensor $T$, include a negative term for every contravariant index, (contract the index with $\Gamma$ as above), and a positive term (as above) for every covariant index in $T$. We say that $T$ is parallel along a curve with tangent vector $X$ if $\nabla_{X} T=0$ all along the curve. It follows that $\nabla_{X} T$ measures the rate at which $T$ diverges from the parallel translation of $T$ in direction $X$. For a $(2,0)$ tensor $T$ we define the covariant divergence of $T$ to be the vector field defined by

$$
\operatorname{div} T=T_{; \sigma}^{i \sigma} \frac{\partial}{\partial x^{i}} .
$$

The covariant derivative commutes with contraction and the raising and lowering of indices, [42], and by (2.12), $\nabla$ reduces to the classical derivative at any point where the Christoffel symbols $\Gamma_{j k}^{i}$ vanish.

It follows from (2.10) that $\Gamma_{j k}^{i}=0$ at a point in a coordinate system where $g_{i j, k}=0$, all $i, j, k=0, \ldots, 3$. The existence of such coordinate frames at a point follows directly from the fact that the metric components $g_{i j}$ are smoothly varying, and transform like a symmetric bilinear form under coordinate transformation. If in addition, $g_{i j}=\operatorname{diag}(-1,1,1,1)$, then such a coordinate system is said to be locally inertial, or locally Lorentzian at the point. The notion of geodesics and parallel translation have a very natural physical interpretation in General Relativity in terms of the locally inertial coordinate frames. Indeed, General Relativity makes contact with (the flat spacetime theory of) Special Relativity by identifying the locally Lorentzian frames at a point as the "locally non-rotating" inertial coordinate systems in which spacetime behaves as if it were locally flat. Thus physically, the non-rotating vector fields carried by an observer in freefall should be the vector fields that are locally constant in the locally inertial coordinate frames defined at each point along the curve. But since $\Gamma_{j k}^{i}=0$ at the center of a locally intertial coordinate system, it follows from (2.11) that a vector field is 
parallel translated along a curve (in the sense that $\nabla_{X} Y=0$ along a curve), if and only if its components are (locally) constant in the locally inertial coordinate frames defined at each point along the curve. Thus we see that the non-rotating vector fields carried by an observer in freefall are exactly the vectors that are parallel transported by the unique symmetric connection (2.10) determined by the gravitational metric $g$. Similarly, the geodesics of the metric $g$ are just the curves that are "locally straight lines" in the locally inertial coordinate frames.

The fundamental tenet of General Relativity is the principle that there is no apriori global inertial coordinate system on spacetime. Rather, in General Relativity, inertial coordinate systems are local properties of spacetime in the sense that they change from point to point. For example, if there were a global Newtonian absolute space, then there would exist global coordinate systems in which freefalling objects do not accelerate, and any two such coordinate systems would be related by transformations from the 10 parameter Galilean Group-the set of coordinate transformations that do not introduce accelerations. In Special Relativity, the existence of absolute space would presume the existence of global coordinate systems related by the transformations of Special Relativity; that is, in Special Relativity, the 10 parameter Poincare group replaces the 10 parameter Galilean Group as the set of transformations that introduce no accelerations. The Poincare Group is obtained from the Galilean group by essentially replacing Euclidean translation in time by Lorentz transformations, and this accounts for time dilation. The spacetime metric can then be viewed as a book-keeping device for keeping track of the location of the local inertial reference frames as they vary from point to point in a given coordinate system-the metric locates the local inertial frames at a given point as those coordinate systems that diagonalize the metric at that point, $g_{i j}=\operatorname{diag}(-1,1,1,1)$, such that the derivatives of the metric components also vanish at the point. Thus, the earth moves "unaccelerated" in each local inertial frame, but these frames change from point to point, thus producing apparent accelerations in a global coordinate system in which the metric is not everywhere diagonal. The fact that the earth moves in a periodic orbit around the Sun is proof that there is no coordinate system that globally diagonalizes the metric, and this is an expression of the fact that gravitational fields produce non-zero spacetime curvature. Indeed, in an inertial coordinate frame, when a gravitational field is present, one cannot in general eliminate the second derivatives of the metric components at a 
point by any coordinate transformation, and the nonzero second derivatives of the metric that cannot be eliminated, represent the gravitational field. These second derivatives are measured by the Riemann Curvature Tensor associated with the Riemannian metric $g$.

Riemann introduced the curvature tensor in his inaugural lecture of 1854 . In this lecture he solved the longstanding open problem of describing curvature in surfaces of dimension higher than two. Although the curvature tensor was first developed for positive definite "spatial" metrics, Einstein accounted for time dilation by letting Lorentz transformations play the role of rotations in Riemann's theory, and except for this, Riemann's theory carries over essentially unchanged. The Riemann Curvature Tensor $R_{j k l}^{i}(\mathbf{x})$ is a quantity that involves second derivatives of $g_{i j}(\mathbf{x})$, but which transforms like a tensor under coordinate transformation; that is, the components transform like a sort of four component version of a vector field, even though vector fields are constructed essentially from first order derivatives. The connection between General Relativity and geometry can be summarized in the statement that the Riemann Curvature Tensor associated with the metric $g$ gives an invariant description of gravitational accelerations. The components of the Riemann Curvature Tensor are given in terms of the Christoffel symbols by the formula, [41],

$$
R_{j k l}^{i}=\Gamma_{j l, k}^{i}-\Gamma_{j k, l}^{i}+\left\{\Gamma_{j l}^{\sigma} \Gamma_{\sigma k}^{i}-\Gamma_{j k}^{\sigma} \Gamma_{\sigma l}^{i}\right\}
$$

One can interpret this as a "curl" plus a "commutator".

\subsection{Introduction to the Einstein Equations}

Once one makes the leap to the idea that the inertial coordinate frames change from point to point in spacetime, one is immediately stuck with the idea that, since our non-rotating inertial frames here on earth are also nonrotating with respect to the fixed stars, the stars must have had something to do with the determination of our non- accelerating reference frames here on earth, (Mach's Principle). Indeed, not every Lorentzian metric can describe a gravitational field, which means that gravitational metrics must satisfy a constraint that describes how inertial frames at different points of spacetime interact and evolve. In Einstein's theory of gravity, this constraint is given 
by the Einstein gravitational field equations. These field equations were first introduced by Albert Einstein in 1915 after almost ten years of struggle.

The Einstein equations can be written in the compact form

$$
G=\kappa T \text {. }
$$

Here $\mathrm{G}$ denotes the Einstein curvature tensor, $T$ the stress energy tensor, (the source of the gravitational field), and $\kappa$ is the gravitational constant. In a given coordinate system $x$, the field equations (2.15) take the component form

$$
G_{i j}(x)=\kappa T_{i j}(x)
$$

where

$$
G_{i j} \equiv R_{i \sigma j}^{\sigma}-\frac{1}{2} R_{\sigma \tau}^{\sigma \tau} g_{i j}
$$

denotes the $x$-components of the Einstein curvature tensor, and $T_{i j}$ the $\mathrm{x}$ components of the stress energy tensor. We let $0 \leq i, j \leq 3$ refer to components in a given coordinate system, and again we assume the Einstein summation convention whereby repeated up-down indices are assumed to be summed from 0 to 3 . The components of the stress energy tensor give the energy density and $i$-momentum densities and their fluxes at each point of spacetime. When the sources are modeled by a perfect fluid, $T$ is given (in contravariant form) by

$$
T^{i j}=(\rho+p) w^{i} w^{j}+p g^{i j},
$$

where $\mathbf{w}$ denotes the unit 4 -velocity vector of the fluid, (the tangent vector to the world line of the fluid particle), $\rho$ denotes the energy density, (as measured in the inertial frame moving with the fluid), and $p$ denotes the fluid pressure. The four velocity $\mathbf{w}$ has components $w^{i}=\frac{d x^{i}}{d s}$ when the fluid particle traverses a (timelike) path $x(s)$ in $x$-coordinates, and $s$ is taken to be the arclength parameter (2.1) determined by the gravitational metric $g$. It follows that $\mathbf{w}$ is a unit timelike vector relative to $g$, and thus only three of the four components of $\mathbf{w}$ are independent. The constant $\kappa$ in (2.15) is determined by the principle that the theory should incorporate Newton's 
theory of gravity in the limit of low velocities and weak gravitational fields, (Correspondence Principle). This leads to the value

$$
\kappa=8 \pi \mathcal{G} / c^{4} .
$$

Again, $c$ denotes the speed of light and $\mathcal{G}$ denotes Newton's gravitational constant.

Newton's constant first appears in the inverse square force law

$$
\text { Force }=M \mathbf{a}=-\mathcal{G} \frac{M M_{0}}{r^{3}} \mathbf{r} .
$$

In (2.19), $M$ is the mass of a planet, $M_{0}$ is the mass of the sun, and $\mathbf{r}$ is the position vector of the planet relative to the center of mass of the system. The Newtonian law (2.19) starts looking like it isn't really a "fundamental law" once one verifies that the inertial mass $M$ on the LHS of (2.19) is equal to the gravitational mass $M$ on the RHS of (2.19), (Equivalence Principle). In this case, $M$ cancels out, and then (2.19) (remarkably) becomes more like a law about accelerations than a law about "forces". That is, once $M$ cancels out, the force law (2.19) is independent of any properties of the object (planet) whose motion it purports to describe. Thus, in Newton's theory, the "gravitational force", which is different on different objects of different masses, miraculously adjusts itself perfectly so that every object, (subject to the same initial conditions), traverses exactly the same path. Thus Einstein was led to suspect that the Newtonian gravitational force was some sort of artificial device, and that the fundamental objects of the gravitational field were the "freefall paths", not the forces. From this point of view, the field equations (2.15) are more natural than (2.19) because they are, at the start, equations for the gravitational metric, and the gravitational metric fundamentally describes the paths of "freefalling" objects by means of the geodesic equation of motion (2.9) — which just expresses "local nonacceleration in locally inertial coordinate frames". In Newton's theory of gravity, the non-rotating frames here on earth are aligned with the stars because there is a global inertial coordinate system that connects us. In contrast, according to the modern theory of cosmology, which is based on Einstein's theory of gravity, the non-rotating inertial frames here on earth are aligned with the stars because the FRW metric (2.15) maintains this alignment, and (2.15) solves the Einstein equations for an appropriate choice of $R(t)$ ). (This is still a bit unsatisfying!) 
In the limit that a finite set of point masses tends to a continuous mass distribution with density $\rho$, Newton's force law is replaced by the Poisson equation for the gravitational potential $\phi$,

$$
-\Delta \phi=4 \pi \mathcal{G} \rho
$$

Indeed, in the case of a compactly supported density $\rho(x)$, one can use the fundamental solution of Laplacian to write the solution of (2.20) as

$$
\phi(x)=\int_{R^{3}} \frac{\mathcal{G}}{|x-y|} \rho(y) d^{3} y,
$$

so the Newtonian acceleration at a point $x$ is given by

$$
\mathbf{a}=-\nabla \phi=\int_{R^{3}} \frac{\mathcal{G}}{|x-y|^{3}}(x-y) \rho(y) d^{3} y .
$$

Thus we recover (2.19) from (2.22) by approximating $\rho$ in $(2.22)$ by a finite number of point masses.

The Einstein equations play the same role in General Relativity that the Poisson equation (2.20) plays in the Newtonian theory of gravity - except there is a very significant difference: the Poisson equation determines the (scalar) gravitational potential $\phi$ given the mass density $\rho$, but in Newton's theory this must be augmented by some system of conservation laws in order to describe the time evolution of the mass density $\rho$ as well. For example, if we assume that the density evolves according to a perfect fluid with pressure $p$ and 3-velocity $\mathbf{v}$, then the coupling of Newton's law of gravity with the Euler equations for a perfect fluid leads to the Euler-Poisson system

$$
\begin{gathered}
\rho_{t}+\operatorname{div}(\rho \mathbf{v})=0, \\
\left(\rho v^{i}\right)_{t}+\operatorname{div}\left(\rho v^{i} \mathbf{v}+p \mathbf{e}^{i}\right)=-\rho \nabla \phi, \\
-\Delta \phi=4 \pi \mathcal{G} \rho .
\end{gathered}
$$

The first four equations are the compressible Euler equations with the gravitational forcing term on the RHS. The first equation, the continuity equation, expresses conservation of mass, the next three express conservation of $i$-momentum, $i=1,2,3$, (for a perfect fluid this really says that the time rate 
of change of momentum is equal to the sum of the force of the pressure gradient plus the force of the gravitational field; $\mathbf{e}^{i}$ denotes the $i$ 'th unit vector in $R^{3}$ ), and the last equation expresses the continuum version of Newton's inverse square force law. Note that for the fluid part of (2.23), information propagates at the sound (and shock) speeds, but the gravitational potential $\phi$ is updated "instantaneously", depending only on the density $\rho(x, t)$, according to the formula (2.21). In contrast, for Einstein's theory of gravity, the time evolution of the gravitational metric is determined simultaneously with the time evolution of the sources through system (2.15), and all of the components of the stress tensor directly influence the components of the gravitational field $g_{i j}$. This principle is the basis for the discovery of the Einstein equations. Indeed, since the 0-column of the stress-energy tensor (2.18) gives the energy and momentum densities, and the $i$-column gives the corresponding $i$-fluxes, (in the relativistic sense), it follows that conservation of energy-momentum in curved spacetime reduces to the statement

$$
\operatorname{Div}(T)=0,
$$

where (capital) Div denotes the covariant divergence for the metric $g$, so that it agrees with the ordinary divergence in each local inertial coordinate frame. In this way equations (2.24) reduce to the relativistic compressible Euler equations in flat Minkowski spacetime. Since the covariant derivative depends on the metric components, the conservation equation (2.24) is essentially coupled to the equation for the gravitational field $g$. But the stress tensor $T$ is symmetric, $T_{i j}=T_{j i}$, and so the tensor on the LHS of (2.16) must also be symmetric, and therefore the Einstein equations (2.16) supply ten independent equations in the ten independent unknown metric components $g_{i j}$, together with the four independent functions among $\rho$ and the unit vector field $\mathbf{w}$. (Here $p$ is assumed to be determined by an equation of state.) But (2.16) assumes no coordinate system, and thus in principle we are free to give four further relations that tie the components of $G$ and $T$ to the coordinate system. This leaves ten equations in ten unknowns, and thus there are no further constraints allowable to couple system (2.15) to the conservation laws (2.24). The only way out is to let (2.24) follow as an identity from (2.15), and this determines the LHS of (2.15), namely, the Einstein tensor $G_{i j}$ is the simplest tensor constructable from $R_{j k l}^{i}$ such that (2.24) follows identically from the Bianchi identities of Riemannian geometry, $\left(R_{j[k l, m]}^{i}=0\right.$, where 
$[k l, m]$ denotes cyclic sum, c.f., [42]). ${ }^{4}$ Thus, the simplest and most natural field equations of form (2.15) are uniquely determined by the equation count, [42]. The next simplest tensor for the LHS of (2.15) that meets (2.24) is

$$
G_{i j}+\Lambda g_{i j},
$$

for constant $\Lambda$. In these notes we always assume $\Lambda=0$. One can show that in the limit of low velocities and weak gravitational fields, the equations (2.24) reduce to the first four equations of $(2.23)$, and the $(0,0)$ component of the Einstein equations (2.16) reduces to the Poisson equation (2.20), thus fixing the choice $\kappa=8 \pi \mathcal{G} / c^{4},[42]$. This establishes the correspondence of Einstein's theory of gravity with the Newtonian theory.

\subsection{Shock Waves in General Relativity and the Ein- stein equations in Schwarzschild Coordinates}

In Einstein's theory of gravity, based on (2.15), the conservation of energy and momentum (2.24) are not imposed, but follow as differential identities from the field equations (2.15). In a specified system of coordinates, (2.15) determines a hyperbolic system of equations that simultaneously describes the time evolution and interaction of local inertial coordinate frames, as well as the time evolution of the fluid according to (2.24). Since GR is coordinate independent, we can always view the time evolution (2.15) in local inertial coordinates at any point in spacetime, in which case (2.24) reduces to the classical relativistic Euler equations at the point. This tells us that, heuristically, shock-waves must form in the time evolution of (2.15) because one could in principle drive a solution into a shock while in a neighborhood where the equations remained a small perturbation of the classical Euler equations. (This is much easier to say than to demonstrate rigorously, and as far as we know, such a demonstration remains a challenging task.) We conclude from this that shock-waves are as fundamental to the time evolution of solutions of the Einstein equations for a perfect fluid, as they are for the time evolution of the classical compressible Euler equations (2.23).

\footnotetext{
${ }^{4}$ This is the simplest known route to the field equations (2.15). Of course, since (2.15) represents a new starting point, it follows that there must be a "conceptual leap" at some stage of any "derivation" of (2.15).
} 
At a shock wave, the fluid variables $\rho, \mathbf{w}$ and $p$ are discontinuous. Notice that (2.15) implies that the Einstein curvature tensor $G$ will be discontinuous at any point where $T$ is discontinuous. Since $G$ involves second derivatives of the metric tensor $g$, the only way (2.15) can hold in the classical pointwise a.e. sense at the shock is if the component functions $g_{i j}$ are continuously differentiable at the shock, with bounded derivatives on either side, that is, if $g_{i j} \in C^{1,1}$. Thus we expect from (2.15) that the spacetime metric $g$ should be $C^{1,1}$ at shock waves. However, we now show that for a spherically symmetric metric in standard Schwarzschild coordinates (2.8), the best one can expect is that $g \in C^{0,1}$.

Using MAPLE to put the metric ansatz (2.8) into the Einstein equations (2.15) produces the following system of four coupled partial differential equations, (c.f. (3.20)-(3.23) of [9]),

$$
\begin{aligned}
\frac{A}{r^{2} B}\left\{r \frac{B^{\prime}}{B}+B-1\right\} & =\kappa A^{2} T^{00}, \\
-\frac{B_{t}}{r B} & =\kappa A B T^{01} \\
\frac{1}{r^{2}}\left\{r \frac{A^{\prime}}{A}-(B-1)\right\} & =\kappa B^{2} T^{11}, \\
-\frac{1}{r A B^{2}}\left\{B_{t t}-A^{\prime \prime}+\Phi\right\} & =\frac{2 \kappa r}{B} T^{22},
\end{aligned}
$$

where the quantity $\Phi$ in the last equation is,

$$
\begin{aligned}
\Phi=-\frac{B A_{t} B_{t}}{2 A B}-\frac{B}{2}\left(\frac{B_{t}}{B}\right)^{2} & -\frac{A^{\prime}}{r}+\frac{A B^{\prime}}{r B} \\
+ & \frac{A}{2}\left(\frac{A^{\prime}}{A}\right)^{2}+\frac{A}{2} \frac{A^{\prime}}{A} \frac{B^{\prime}}{B} .
\end{aligned}
$$

Here "prime" denotes $\partial / \partial r$, and again $\kappa=\frac{8 \pi \mathcal{G}}{c^{4}}$ is the coupling constant, $\mathcal{G}$ is Newton's gravitational constant, $c$ is the speed of light, $T^{i j}, i, j=0, \ldots, 3$ are the components of the stress energy tensor, and $A \equiv A(r, t), B \equiv B(r, t)$ denote the components of the gravitational metric tensor (2.8) in standard Schwarzschild coordinates $\mathbf{x}=\left(x^{0}, x^{1}, x^{2}, x^{3}\right) \equiv(t, r, \theta, \phi)$. The mass function $M$ is defined through the identity 


$$
B=\left(1-\frac{2 M}{r}\right)^{-1} .
$$

In terms of the variable $M$, equations (2.25) and (2.26) are equivalent to

$$
M^{\prime}=\frac{1}{2} \kappa r^{2} A T^{00}
$$

and

$$
\dot{M}=-\frac{1}{2} \kappa r^{2} A T^{01},
$$

respectively. Using the perfect fluid assumption (2.18), the components $T^{i j}$ satisfy

$$
\begin{aligned}
T^{00} & =\frac{1}{A} T_{M}^{00}, \\
T^{01} & =\frac{1}{\sqrt{A B}} T_{M}^{01}, \\
T^{11} & =\frac{1}{B} T_{M}^{11},
\end{aligned}
$$

where $T_{M}^{i j}$ denote the components of $T$ in flat Minkowski spacetime. The components of $T_{M}$ are given by

$$
\begin{aligned}
T_{M}^{00} & =\frac{c^{4}+\sigma^{2} v^{2}}{c^{2}-v^{2}} \rho, \\
T_{M}^{01} & =\frac{c^{2}+\sigma^{2}}{c^{2}-v^{2}} c v \rho, \\
T_{M}^{11} & =\frac{v^{2}+\sigma^{2}}{c^{2}-v^{2}} \rho c^{2},
\end{aligned}
$$

where $\sigma^{2}=p / \rho$, c.f., $[28,9]$. Here $v$, taken in place of $\mathbf{w}$, denotes the fluid velocity as measured by an observer fixed with respect to the radial coordinate $r$. It follows from (2.30) together with (2.35)-(2.37) that, if $r \geq$ $r_{0}>0$, then

$$
M(r, t)=M\left(r_{0}, t\right)+\frac{\kappa}{2} \int_{r_{0}}^{r} T_{M}^{00}(r, t) r^{2} d r
$$


and it follows directly form (2.35)-(2.37) that

$$
\begin{gathered}
\left|T_{M}^{01}\right|<T_{M}^{00}, \\
\sigma^{2} c^{2}+(p / \rho) T_{M}^{00}<T_{M}^{11}<T_{M}^{00},
\end{gathered}
$$

so long as $\sigma<c$. Equation (2.38) shows that $M(r, t)$ can be interpreted as the total mass inside radius $r$ at time $t$.

Now we are interested in solutions of (2.25)-(2.28) in the case when shock waves are present. A shock wave in the compressible Euler equations leads to discontinuities in the fluid density, pressure and velocity, and thus in light of $(2.18)$, it follows that a shock wave would produce a discontinuity in the stress tensor $T$ at a shock. But when $T$ is discontinuous, equations (2.25)(2.27) above imply immediately that derivatives of the metric components $A$ and $B$ are discontinuous at shocks. Moreover, if $A$ and $B$ have discontinuous derivatives when shock waves are present, it follows that (2.28), being second order, cannot hold classically, and thus equation (2.28) must be taken in the weak sense, that is, in the sense of the theory of distributions. From these considerations, we see that the metric components $A$ and $B$ can be at best only Lipschitz continuous, that is, $C^{0,1}$, at shock waves. That is, $A$ and $B$ are one degree less smooth than the general theory suggests they should be, [11].

The general problem of making sense of gravitational metrics that are only Lipschitz continuous at shock surfaces was taken up in [30]. The analysis there identifies conditions that must be placed on the metric in order to ensure that conservation holds at the shock, and that there do not exist delta-function sources at the shock, [12]. When these conditions are met, the methods in [30] imply the existence of a $C^{1,1}$ coordinate transformation, (to Gaussian normal coordinates), that improves the level of smoothness of the metric components from $C^{0,1}$ up to $C^{1,1}$ at the shock. All of this is the subject of Subsection 2.1. However, these results apply only to smooth interfaces that define a single shock surface for which $G=\kappa T$ holds identically on either side. For general shock wave solutions of (2.25)-(2.28), (that can contain multiplicities of interacting shock waves), it is an open question whether there exists a coordinate transformation that can increase the level of smoothness of the metric components by one order, because the Gaussian normal coordinate system for the shock surface breaks down at points where shock waves interact. 
We conclude this section by showing that the mapping $(r, t) \rightarrow(\bar{r}, \bar{t})$ that takes an arbitrary metric of form (2.7) over to one of form (2.8), implies a loss of one order of differentiability in the metric components when shock waves are present. This argues that our results are consistent with the existence of such a smoothing coordinate transformation, but still leaves open the problem of the existence of such a transformation.

We review the construction of the mapping $(r, t) \rightarrow(\bar{r}, \bar{t}),[42,9]$ with an eye toward keeping track of the smoothness class of the metric at each stage. To start, one must assume that the metric component $C(t, r)$ in $(2.7)$ satisfies the condition that for each fixed $t, C$ increases from zero to infinity as $r$ increases from zero to infinity, and that

$$
\frac{\partial}{\partial r} C(r, t) \neq 0
$$

(These are not unreasonable assumptions considering that $C$ measures the areas of the spheres of symmetry.) Define

$$
\bar{r}=\sqrt{C(r, t)} .
$$

Then the determinant of the Jacobian of the mapping $(r, t) \rightarrow(\bar{r}, t)$ satisfies

$$
\left|\frac{\partial \bar{r}}{\partial r}\right|=\frac{\partial}{\partial r} \sqrt{C(r, t)} \neq 0
$$

in light of (2.41). Thus the transformation to $(\bar{r}, t)$ coordinates is (locally) a nonsingular transformation, and in $(\bar{r}, t)$ coordinates the metric $(2.7)$ takes the form

$$
d s^{2}=-A(r, t) d t^{2}+B(r, t) d r^{2}+2 E(r, t) d t d \bar{r}+r^{2} d \Omega^{2} .
$$

(Here we have replaced $\bar{r}$ by $r$ and $A, B$ and $E$ stand in for the transformed components.) It is easy to verify that, to eliminate the mixed term, it suffices to define the time coordinate $\bar{t}$ so that, cf. [42],

$$
d \bar{t}=\phi(r, t)\{A(r, t) d t-E(r, t) d r\} .
$$

In order for (2.44) to be exact, so that $\bar{t}$ really does define a coordinate function, the integrating factor $\phi$ must be chosen to satisfy the (linear) PDE 


$$
\frac{\partial}{\partial r}\{\phi(r, t) A(r, t)\}=-\frac{\partial}{\partial t}\{\phi(r, t) E(r, t)\} .
$$

But we can solve $(2.45)$ for $\phi(r, t)$ from initial data $\phi\left(r, t_{0}\right)$, by the method of characteristics. From this it follows that, (at least locally), we can transform metrics of form (2.7) over to metrics of form (2.8) by coordinate transformation. To globalize this procedure, we need only assume that $C_{r}(t, r) \neq 0$, and that $C$ takes values from zero to infinity at each fixed $t$. Now note that in general $\phi(r, t)$, the solution to $(2.45)$, will have the same level of differentiability as $A(r, t)$ and $E(r, t)$; and so it follows that the components of $d t$ and $d r$ in (2.44) will have this same level of differentiability. This implies that the $\bar{t}$ transformation defined by (2.44) preserves the level of smoothness of the metric component functions. On the other hand, the $\bar{r}$ transformation in (2.42) reduces the level of differentiablility of the metric components by one order. Indeed, the level of smoothness of the transformed metric component functions are in general no smoother than the Jacobian that transforms them, and by (2.42), the Jacobian of the transformation contains the terms $C_{r}$ and $C_{t}$ which will in general be only $C^{0,1}$ when $C \in C^{1,1}$. Thus, if we presume, (motivated by [29]), that for general spherically symmetric shock wave solutions of $G=\kappa T$, that there exists a coordinate system in which the metric takes the form (2.7), and the components of $g$ in these coordinates are $C^{1,1}$ functions of these coordinates, then it follows that we cannot expect the transformed metrics of form (2.8) to be better than $C^{0,1}$, that is, Lipschitz continuous.

In Section 5 we show that when $A$ and $B$ are Lipschitz continuous functions of $(t, r)$, and $T$ is bounded in $L^{\infty}$, system (2.25)-(2.28) is weakly equivalent to the system obtained by replacing (2.26) and (2.28) by the system $D i v T=0$ in the form,

$$
\begin{aligned}
\left\{T_{M}^{00}\right\}_{, 0}+\left\{\sqrt{\frac{A}{B}} T_{M}^{01}\right\}_{, 1}= & -\frac{2}{x} \sqrt{\frac{A}{B}} T_{M}^{01} \\
\left\{T_{M}^{01}\right\}_{, 0}+\left\{\sqrt{\frac{A}{B}} T_{M}^{11}\right\}_{, 1}= & -\frac{1}{2} \sqrt{\frac{A}{B}}\left\{\frac{4}{x} T_{M}^{11}+\frac{(B-1)}{x}\left(T_{M}^{00}-T_{M}^{11}\right)\right. \\
& \left.+2 \kappa x B\left(T_{M}^{00} T_{M}^{11}-\left(T_{M}^{01}\right)^{2}\right)-4 x T^{22}\right\}
\end{aligned}
$$


(We use $x$ in place of $r$ when the equations are expressed as a system of conservation laws.) This is a nice formulation of DivT $=0$ because the conserved variables $u=\left(T_{M}^{00}, T_{M}^{01}\right)$ are the Minkowski energy and momentum densities, (c.f. (2.35), (2.36)), and thus do not depend on the metric components $\mathbf{A} \equiv(A, B)$. Note that all terms involving $A_{r}, B_{r}$ and $B_{t}$ in the equation $\operatorname{DivT}=0$ have been eliminated by substitution using equations $(2.25),(2.26)$ and $(2.27)$. However, DivT $=0$ also contains terms that involve $A_{t}=0$, and there is no $A_{t}$ equation among (2.25)-(2.28) - so some change of variables is required to eliminate such terms from DivT $=0$ in order to close the equations, (c.f. (6.21), (6.22) below). It turns out that it suffices to choose $T_{M}^{00}$ and $T_{M}^{01}$ as independent variables; that is, when we substitute for $T_{M}^{00}$ and $T_{M}^{01}$ in favor of the original conserved quantities $T^{00}$ and $T^{01}$, all terms involving $A_{t}$ in $\operatorname{DivT}=0$, , (remarkably), cancel out, thus allowing the formulation (2.46), (2.47).

When $T^{11}$ and $T^{22}$ are expressed in terms of $u=\left(u^{0}, u^{1}\right) \equiv\left(T_{M}^{00}, T_{M}^{01}\right)$ in (2.46), (2.47), (2.25) and (2.27), the equations close, and what results is a system of conservation laws with source terms that takes the compact form

$$
\begin{gathered}
u_{t}+f(\mathbf{A}, u)_{x}=g(\mathbf{A}, u, x), \\
\mathbf{A}^{\prime}=h(\mathbf{A}, u, x) .
\end{gathered}
$$

The first equation in $(2.49)$ is $(2.46),(2.47)$, and the second equation is $(2.25),(2.27)$, so that

$$
\begin{aligned}
u & =\left(T_{M}^{00}, T_{M}^{01}\right) \equiv\left(u^{0}, u^{1}\right) \\
\mathbf{A} & =(A, B), \\
f(\mathbf{A}, u) & \equiv\left(f^{0}, f^{1}\right)=\sqrt{\frac{A}{B}}\left(T_{M}^{01}, T_{M}^{11}\right),
\end{aligned}
$$

and $g=\left(g^{0}, g^{1}\right)$ is determined from the RHS of (2.46), (2.47), while $h=$ $\left(h^{0}, h^{1}\right)$ is determined from the RHS of $(2.27)$, (2.25) upon solving for $\left(A^{\prime}, B^{\prime}\right)$, respectively, (c.f. (6.49) below). Note that (2.48), (2.49) do indeed allow for $C^{0,1}$ metrics with discontinuous density and velocity based on the conservation law structure of these equations, and such solution correspond to gravitational metrics that are in the smoothness class $C^{0,1}$. In general, they do not admit solution metrics smoother than Lipschitz continuous. 
Solutions to equations (2.48), (2.49) have recently been constructed in [10] by a fractional step Glimm scheme that is locally inertial. The main result of that work can be stated as follows, (we refer to [10]) for details):

Assume that

$$
p=\sigma^{2} \rho,
$$

where $\sigma$, the sound speed, is assumed to be constant, $\sigma<c$. (Examples of this, including the case $\sigma^{2}=1 / 3$, and the case of an isothermal sphere, are important physically, but here we view (2.50) as a natural model problem for general relativity because (2.50) keeps wave speeds subluminous, and prevents the formation of vacuum states, [10]. The assumption of spherical symmetry together with (2.50) defines the simplest possible setting for shock wave propagation in the Einstein equations.) The assumption (2.50) implies that the scalar curvature $R$ is proportional to the density,

$$
R=\left(c^{2}-3 \sigma^{2}\right) \rho .
$$

For the existence theorem, assume the initial boundary conditions

$$
\begin{aligned}
& \rho(r, 0)=\rho_{0}(r), \quad v(r, 0)=v_{0}(r), \quad \text { for } r>r_{0}, \\
& M\left(r_{0}, t\right)=M_{r_{0}}, \quad v\left(r_{0}, t\right)=0, \quad \text { for } t \geq 0,
\end{aligned}
$$

where $r_{0}$ and $M_{r_{0}}$ are positive constants, and assume the no black hole and finite total mass conditions,

$$
\frac{2 M(r, t)}{r}<1, \quad \lim _{r \rightarrow \infty} M(r, t)=M_{\infty}<\infty,
$$

hold at $t=0$. For convenience, assume further that

$$
\lim _{r \rightarrow \infty} r^{2} T_{M}^{00}(r, t)=0,
$$

holds at $t=0$. The main result of [10] can be stated as follows:

Theorem 1 Assume that the initial boundary data satisfy (2.52)-(2.54), and assume that there exist positive constants $L, V$ and $\bar{v}$ such that the initial velocity and density profiles $v_{0}(r)$ and $\rho_{0}(r)$ satisfy 


$$
T V_{[r, r+L]} \ln \rho_{0}(\cdot)<V, \quad T V_{[r, r+L]} \ln \left(\frac{c+v_{0}(\cdot)}{c-v_{0}(\cdot)}\right)<V, \quad\left|v_{0}(r)\right|<\bar{v}<c,
$$

for all $r_{0} \leq r<\infty$, where $T V_{[a, b]} f(\cdot)$ denotes the total variation of the function $f$ over the interval $[a, b]$. Then a bounded weak (shock wave) solution of (2.25)-(2.28), satisfying (2.52) and (2.53), exists up to some positive time $T>0$. Moreover, the metric functions $A$ and $B$ are Lipschitz continuous functions of $(r, t)$, and (2.55) continues to hold for $t<T$ with adjusted values for $V$ and $\bar{v}$ that are determined from the analysis.

Note that we cannot expect bounded weak solutions for all time $T \rightarrow \infty$ because black holes can form in finite time, and the metric component $B=$ $\left(1-\frac{2 \mathcal{G} M}{r}\right)^{-1} \rightarrow \infty$ at a black hole $r=2 \mathcal{G} M$ in standard Schwarzschild coordinates. By (2.51), the case $\rho \rightarrow \infty$ as $t \rightarrow T$ would correspond to the formation of a naked singularity. Note that by (2.25) and (2.27), the metric components $A$ and $B$ will be no smoother than Lipschitz continuous when shocks are present, and since (2.28) is second order in the metric, it follows that (2.28) is only satisfied in the weak sense of the theory of distributions. Note finally that (2.53) says that the total mass is constant in $[0, T)$, consistent with the conclusion that there do not exist delta function sources of mass at shock waves, or at points of shock wave interaction, in these solutions.

Theorem 1 confirms what is indicated by equations (2.48) and (2.49): that the Einstein equations are consistent at the level of $C^{0,1}$ metrics, and are meaningful in the presence of arbitrary numbers of interacting shock waves, of arbitrary strength. A careful derivation of (2.48), (2.49) is given in Section 6, but Theorem 1 will not be discused in these notes. The interested reader should consult [10] for a detailed proof of Theorem 1. 


\section{Weak solutions of the Einstein Equations when the Metric is only Lipschitz Contin- uous Across an Interface}

In this section we consider a general four dimensional spacetime manifold with metric tensor $g$ having signature $\eta_{i j}=\operatorname{diag}(-1,1,1,1)$. We look to characterize solutions of the Einstein field equations (2.16) that are only Lipschitz continuous across a smooth 3 -dimensional surface $\Sigma$. To start, recall that,

$$
G_{i j} \equiv R_{i j}-(1 / 2) R g_{i j}
$$

is the Einstein curvature tensor, where $R_{i j}$ and $R$ denote the Ricci curvature tensor and Ricci scalar curvature, respectively, formed from the Riemann curvature tensor of the metric $g$. The Riemann curvature tensor, with components $R_{j k l}^{i}$, is given by

$$
R_{j k l}^{i}=\Gamma_{j k, l}^{i}-\Gamma_{j l, k}^{i}+\Gamma_{\sigma l}^{i} \Gamma_{j k}^{\sigma}-\Gamma_{\sigma k}^{i} \Gamma_{j l}^{\sigma},
$$

and $R_{i j}$ and $R$ are obtained by the contractions

$$
R_{i j}=R_{i \sigma j}^{\sigma}
$$

and

$$
R=R_{\sigma}^{\sigma}
$$

The Einstein tensor $G$ satisfies the condition $\operatorname{div} G=0$, where $\operatorname{div}$ denotes the covariant divergence defined in terms of the covariant derivative $\nabla$ of the metric connection for $g$. We reiterate that since $\operatorname{div} G=0$, it follows that for solutions of (2.16) we must have $\operatorname{div} T=0$. The distinction here is that $\operatorname{div} G=0$ is a geometric identity, independent of the Einstein equations, and holds as a consequence of the Bianchi identities, while divT $=0$ relies on both the identity $\operatorname{div} G=0$ as well as the Einstein equations (2.16). In later

sections we will assume the stress tensor for a perfect fluid, which is given in covariant components as

$$
T_{i j}=\left(p+\rho c^{2}\right) u_{i} u_{j}+p g_{i j}
$$


In the case of a barotropic equation of state, $p$ is assumed to be given by a function of $\rho$ alone, $p=p(\rho)$. In this case, $\operatorname{div} T=0$ gives four additional equations which hold on solutions of (2.16). In the case when shock-waves are present, the Rankine-Hugoniot jump conditions

$$
\left[T_{i j}\right] n^{i}=0, \quad j=0, \ldots, 3 .
$$

express the weak formulation of conservation of energy and momentum across shock surfaces, [28]. On solutions of the Einstein equations, (3.4) follows from the jump conditions

$$
\left[G_{i j}\right] n^{i}=0, \quad j=0, \ldots, 3 .
$$

(From here on, [.] always denotes the jump in a quantity on either side of an interface.) The jump condition (3.4) involves the fluid variables, but the jump condition (3.5) is independent of the fluid variable, and involves the metric tensor $g$ alone. In the following sections we will generalize the OppenheimerSnyder model by matching two (metric) solutions of the Einstein equations (2.16) in a Lipschitz continuous manner across a spherical shock surface. It is not so easy to verify the Rankine-Hugoniot jump relations (3.4) directly in these examples because (3.4) involves the fluid variables in (3.3), so a direct verification of (3.4) requires using $\operatorname{div} T=0$, which is not an identity, and so cannot be managed without invoking the full Einstein equations (2.16). However, in the next section we bypass this problem with a general theorem which implies that (3.4) follows as a geometric identity from the corresponding identities $\operatorname{div} G=0$ together with geometrical constraints on the second fundamental form on the shock surface, once one knows that the metric is Lipschitz continuous across the shock surface.

The second fundamental form $K: T_{\Sigma} \rightarrow T_{\Sigma}$ on a co-dimension one surface $\Sigma$ with normal vector field $\mathbf{n}$, imbedded in an ambient Riemannian space with metric tensor $g_{i j}$, is a tensor field defined on the surface in terms of the metric $g$, and describes how the surface is imbedded in the ambient spacetime. Here, $T_{\Sigma}$ denotes the tangent space of $\Sigma$. The second fundamental form $K$ is defined by the condition

$$
K(\mathbf{X})=-\nabla_{\mathbf{X}} \mathbf{n}
$$

for $\mathbf{X} \in T_{\Sigma}$. When the metric is only Lipschitz continuous across a codimension one surface, the second fundamental form $K$ is determined separately from the metric values on either side. In the next section we give 
necessary and sufficient conditions (the Israel conditions) for conservation to hold at a Lipschitz continuous shock-wave interface, the condition being given in terms of geometric conditions on the jump in the second fundamental form across the surface. The conditions are that

$$
\begin{gathered}
{\left[\operatorname{tr}\left(K^{2}\right)-(\operatorname{tr} K)^{2}\right]=0,} \\
{[\operatorname{div} K-d(\operatorname{tr} K)]=0,}
\end{gathered}
$$

where $t r$ denotes trace, div denotes covariant divergence, and $d$ denotes exterior differentiation in the surface. We conclude that the physical conservation laws (3.4) turn out to be a consequence of geometrical constraints built apriori into the Einstein tensor, together with geometrical constraints that describe how the shock surface is imbedded in the ambient spacetime manifold. We note that a sufficient condition for conservation is that $[K]=0$ everywhere across the surface. In fact, this implies that in Gaussian normal coordinates the metric will then be in $C^{1}$ because $K_{i j}=g_{i j, n}$ in these coordinates, where, $n$ denotes differentiation in the direction normal to the surface. (See $[12,22,41,42]$.) As we point out in the next section, the transformation to Gaussian normal coordinates is in general only a $C^{1,1}$ coordinate transformation, but once this transformation is made, the $C^{\infty}$ coordinate transformations alone are sufficient to describe the locally Lorentzian properties of the spacetime. (Recall that by $C^{1,1}$ we mean $C^{1}$ with Lipschitz continuous derivatives.) In the case of metrics that are only Lipschitz continuous, the natural class of coordinate transformations is the class of $C^{1,1}$ transformations. Indeed, if the mapping $x \rightarrow y$ is $C^{1,1}$, then $\partial x / \partial y$ and $\partial y / \partial x$ are Lipschitz continuous, and thus Lipschitz continuous tensors are mapped to Lipschitz continuous tensors under the mapping $x \rightarrow y$, and this is the least smooth class of transformations that preserves this mapping. Note that by allowing $C^{1,1}$ transformations, we allow derivatives of $\partial x / \partial y$ and $\partial y / \partial x$ to jump, and this allows us to adjust the jump in the derivatives of tensors across a shock surface. For example, if $g=g^{L} \cup g^{R}$, then

$$
g_{i j}=g_{\alpha \beta} \frac{\partial y^{\alpha}}{\partial x^{i}} \frac{\partial y^{\beta}}{\partial x^{i}},
$$

so the jumps in the derivatives of $\frac{\partial y^{\alpha}}{\partial x^{i}}$ change the jumps in the derivatives of $g_{i j}$ across $\Sigma$, and Israel's result states that within the class of $C^{1,1}$ transformations, we can match the derivatives in $g$ across $\Sigma$ if and only if $[K]=0$, 
the map to Gaussian normal coordinates being $C^{1,1}$. Now in the Einstein equations $G_{i j}=\kappa T_{i j}, G_{i j}$ is the image of a second order differential operator on the metric entries $g_{i j}$, and thus in general we expect metrics that are Lipschitz continuous across $\Sigma$ to have delta function sources in $G$, and hence in the fluid variables $T$, on $\Sigma$. natural to ask, first, when do such delta function sources appear at a shock- wave $\Sigma$ given that the metric is only Lipschitz continuous across $\Sigma$, and second, what is the physical significance of such delta functions sources when they do appear? For the first question, we present a proof in the next section that if $g=g^{L} \cup g^{R}$ is Lipschitz continuous across $\Sigma$ in a coordinate system $x$, then delta function sources appear in $G$ on $\Sigma$ in $x$-coordinates if an only if $[K] \neq 0$, (c.f. $[22]$ ). For the physical interpretation of the delta function sources in $G$, and hence in $T$, when $[K] \neq 0$ at $\Sigma$, we comment that the equivalence of the jump conditions $\left[G_{j}^{i}\right] n_{i}=0=\left[T_{j}^{i}\right] n_{i}$, and the weak formulation of $\operatorname{div} G=0$ at a point $P$ in spacetime is based on the existence of locally Lorentzian coordinate frames at $P$; i.e., coordinates in which $g_{i j, k}(P)=0$. In such coordinate frames, spacetime is locally flat, and the physical principles of special relativity can thus be identified locally. In particular, the covariant divergence agrees with the classical divergence in locally Lorentzian frames, and the global physical conservation laws $\int_{\partial \Omega}=0$ of special relativity can be reduced in local form to $\operatorname{div} T=0$ in curved spacetime. (It is well known that, except in special cases, there do not exist global conservation laws in General Relativity.) In the next section we show that, within the class of $C^{1,1}$ coordinate transformations, there do not exist locally Lorentzian coordinate frames in a neighborhood of a point $P \in \Sigma$ where $G_{i j}$ has a delta function source. Thus, spacetime is not locally flat at points on a Lipschitz continuous shock-wave where $G$ has delta function sources. In the Section 5 we show that for spherically symmetric shock-waves, $\left[G_{j}^{\sigma}\right] n_{\sigma}=0$ implies $[K]=0$, and thus conservation implies that there are no delta function sources in the shock-waves we construct as generalizations of the Oppenheimer-Snyder case, and thus these solutions are locally Lorentzian at each point on the shock. It is an interesting open question as to whether general Lipschitz continuous shocks can evolve from smooth solutions in the time evolution of $G=\kappa T$. 


\subsection{The General Problem}

In this section we give the proof that the jump conditions (3.5) hold at a Lipschitz continuous shock surface if and only if (3.7) and (3.8) hold. We formulate the theorem in $n$-dimensions for a nonsingular metric $g$ of fixed signature $\eta=\operatorname{diag}\left(\epsilon_{1}, \cdots, \epsilon_{n}\right)$ where each $\epsilon_{i}={ }_{-}^{+} 1$. Before stating the theorem, we introduce some notation. Thus let $y \equiv\left(y^{1}, \cdots, y^{n}\right)$ be a smooth coordinate system defined on an $n$-dimensional manifold $M, y: M \rightarrow R^{n}$, and let $\Sigma$ be a smooth hypersurface in $M$. Assume that $\Sigma$ is given locally by $\psi(y)=0$, where $\psi$ is a smooth function satisfying

$$
n_{i} d y^{i} \equiv \frac{\partial \psi}{\partial y^{i}} d y^{i} \neq 0 .
$$

Let $L$ and $R$, (for "left" and "right"), denote the two sides of $M$ defined by the surface $\Sigma$, and let $g^{L}$ and $g^{R}$ denote smooth metrics defined on the left and right side of $\Sigma$, respectively. (It suffices to assume $g^{L}$ and $g^{R}$ are at least $C^{2}$, with derivatives uniformly bounded at $\Sigma$, and we assume this from here on out.) For completeness, we give a proof of the following theorem due to Israel, [12, 22].

Theorem 2 Let $g=g^{L} \cup g^{R}$ denote a nonsingular metric of arbitrary signature whose components $g_{i j}$ in $y$-coordinates are smooth on the left and right sides of $\Sigma$, separately, and Lipschitz continuous across the surface. Assume that $\Sigma$ is given locally by $\varphi=0$, where $\varphi$ is smooth, assume that (3.9) holds, and assume that the normal vector $\mathbf{n}$ is non-null relative to the metric $g$, so that (without loss of generality) we may take $\mathbf{n}$ to be a unit vector $g_{i j} n^{i} n^{j}=1$. Then

$$
\left[G_{j}^{i}(y(P))\right] n_{i}(y(P))=0
$$

at a point $P \in \Sigma$ if and only if both

$$
\left[(\operatorname{tr} K)^{2}-\operatorname{tr}\left(K^{2}\right)\right]=0,
$$

and

$$
[\operatorname{div} K-d(\operatorname{tr} K)]=0,
$$

hold. (Here, the invariant operations div, trace and $d$ on $K$ are restricted to the surface $\Sigma$.) 
Note that by a smooth transformation of the coordinates in a neighborhood of a point $P \in \Sigma$ we may assume that the surface $\Sigma$ is given by $\varphi=y^{n}=0$, so that $\mathbf{n}=\partial / \partial y^{n}$. In this case, the invariant conditions (3.11) and (3.12) reduce in $y$-coordinates to

$$
\left[\left(K_{i}^{i}(y(P))\right)-\left(K_{i}^{i}(y(P))\right)^{2}\right]=0
$$

and

$$
\left[K_{j ; i}^{i}(y(P))-K_{i ; j}^{i}(y(P))\right]=0,,
$$

where the summation in (3.13) and (3.14) is assumed to run from 1 to $n-1$.

The proof of Theorem 2 will follow as a consequence of several lemmas. The idea is to construct Gaussian normal coordinates for the surface $\Sigma$, these being coordinates in which the components of the second fundamental form take the simple form $K_{i j}=-\frac{1}{2} g_{i j, n}$. We then use this identity to write the Einstein curvature tensor $G$ and the jump conditions (3.5) in terms of the $K_{i j}$ and obtain (3.13) and (3.14), [12, 22]. We will use the following identities for the components of the curvature tensor $G_{j}^{i}$ in an arbitrary coordinate system:

Lemma 1 The components of $G$ are given by

$$
\begin{gathered}
G_{i}^{i}=-\sum_{\sigma, \tau \neq i} R_{[\sigma \tau]}^{[\sigma \tau]}, \quad i=1, \cdots, n \\
G_{j}^{i}=\sum_{\tau \neq i, j} R_{[j \tau]}^{[i \tau]}, \quad i \neq j,
\end{gathered}
$$

where the square braces [] around a set of indices indicates that summation is to be taken only over the increasing sequences of indices occurring inside the braces.

Proof: To prove (3.15), we have

$$
G_{j}^{i}=R_{j}^{i}-\frac{1}{2} R \delta_{j}^{i}
$$

But

$$
R_{i}^{i}=R_{\tau i}^{\tau i}=\sum_{\tau \neq i} R_{\tau i}^{\tau i}
$$


because $R_{\gamma \delta}^{\alpha \beta}$ is antisymmetric in $(\alpha \beta)$ and $(\gamma \delta)$. Moreover,

$$
R=R_{\sigma \tau}^{\sigma \tau}=2 R_{[\sigma \tau]}^{[\sigma \tau]}
$$

and so

$$
G_{i}^{i}=R_{[\tau i]}^{[\tau i]}-R_{[\sigma \tau]}^{[\sigma \tau]}=-\sum_{\sigma, \tau \neq i} R^{[\sigma \tau]} .
$$

To prove (3.16) we have

$$
G_{j}^{i}=R_{j}^{i}=R_{\tau j}^{\tau i}=\sum_{\tau \neq i, j} R_{[\tau j]}^{[\tau i]} .
$$

We now construct a Gaussian normal coordinate system $\left(w^{1}, \cdots, w^{n}\right)$ associated with the surface $\Sigma$ in a neighborhood of $P_{0} \in \Sigma$, [41]. To this end, assume that $g$ has $y$-components $g_{i j}$, and by making a smooth coordinate transformation we may assume without loss of generality that $\Sigma$ is defined, (near $P_{0}$ ), by $y^{n}=0$. For each $P \in \Sigma$ let $\gamma_{P}(s)$ denote the geodesic satisfying

$$
\gamma_{P}(0)=P, \quad \dot{\gamma}_{P}(0)=\mathbf{n},
$$

where $\mathbf{n}$ is the normal vector to $\Sigma$ at $P, s$ is arclength, and for convenience we assume that $\mathbf{n}$ points into the right side of $\Sigma$. We define the $w^{n}$-coordinate in a neighborhood of $P_{0} \in \Sigma$ as the "distance from $\Sigma$ " as follows: if $\gamma_{P}(s)=Q$, then set $w^{n}(Q)=s$. In this way, $w^{n}<0$ on the left side of $\Sigma$, and $w^{n}>0$ on the right side of $\Sigma$. Now define the $w^{i}$-coordinates for $i=1, \cdots, n-1$, by $w^{i}(P)=y^{i}(P)$ for $P \in \Sigma$, and define $w^{i}$ in a neighborhood of $\Sigma$ by taking $w^{i}$ to be constant along each $\gamma_{P}(s)$; i.e.,

$$
w^{i}(Q)=w^{i}(P) \text { if and only if } Q=\gamma_{P}(s),
$$

for some $P$ and $s, i=1, \cdots, n$. The coordinates $w=\left(w^{1}, \cdots, w^{n}\right)$ are called Gaussian normal coordinates in a neighborhood of $P_{0} \in \Sigma$. Note that the Gaussian normal coordinates $w$ are in general only $C^{1,1}$ related to the original $y$-coordinates because the geodesics normal to the surface $\Sigma$ are in general only $C^{1}$ curves since the $\Gamma_{j k}^{i}$ can in general have jump discontinuities at $\Sigma$ when $g$ is only Lipschitz continuous across $\Sigma$. (Indeed, to see this, consider the curves $y_{\bar{y}}(s)$ where $\bar{y}=\left(y^{1}, \ldots, y^{n-1}\right)$ and $(\bar{y}, 0) \in \mathbf{R}^{n}$ is the coordinate value of the point $P$ on $\Sigma$ such that $\gamma_{P}(s)=Q$ has $y$-coordinates $y_{\bar{y}}(s)$. Thus, $y(P)=\bar{w}(P)$ for $P \in \Sigma$. But being constructed from families of geodesics on 
each side of $\Sigma, y_{\bar{y}}(s) \equiv \varphi(\bar{y}, s)$ is a smooth function of $\bar{y}$ and $s$ on each side of $\Sigma$ separately. It remains to check continuity of derivatives at $y^{n}=0$. But, at $s=0$,

$$
\frac{\partial y^{i}}{\partial \bar{w}^{j}}=\frac{\partial y_{\bar{y}}^{i}}{\partial \bar{y}^{j}}(s)=\delta_{j}^{i},
$$

because $y=(\bar{y}, 0)$ at $s=0$. Moreover,

$$
\frac{\partial y^{i}}{\partial \bar{w}^{n}}=\frac{\partial y_{\bar{y}}^{i}}{\partial s}(s)=\mathbf{n}^{i}
$$

where $\mathbf{n}^{i}$ denote the $y$-coordiantes of the normal to $\Sigma$ at $P$. Since the metric is continuous at $\Sigma$, this latter derivative is continuous across $\Sigma$ as well.)

Gaussian normal coordinates satisfy the following well-known lemma, $[29]$.

Lemma 2 In Gaussian normal coordinates,

$$
d s^{2}=d\left(w^{n}\right)^{2}+g_{i j} d w^{i} d w^{j},
$$

where the summation on $i$ and $j$ is from 1 to $n-1$.

Note that Lemma 1 implies that the surfaces $w^{n}=$ const. are orthogonal to the coordinate directions $\partial / \partial w^{i}$, for $i=1, \cdots, n-1$.

For a smooth metric $g$, the components of the second fundamental form are given by the following lemma:

Lemma 3 In Gaussian normal coordinates,

$$
K_{i j}=-\frac{1}{2} g_{i j, n}
$$

Proof: We have, for every vector field $X^{i}$,

$$
-K_{\sigma}^{i} X^{\sigma}=\left(\nabla_{\mathbf{X}} \mathbf{n}\right)^{i}=\mathbf{n}_{, \sigma}^{i} X^{\sigma}+\Gamma_{\sigma n}^{i} X^{\sigma}=\Gamma_{\sigma n}^{i} X^{\sigma}
$$

so that

$$
K_{\sigma}^{i} X^{\sigma}=-\Gamma_{\sigma n}^{i} X^{\sigma}
$$

But 


$$
\Gamma_{\sigma n}^{i}=\frac{1}{2} g^{i \tau}\left\{-g_{\sigma n, \tau}+g_{\tau \sigma, n}+g_{n \tau, \sigma}\right\}=\frac{1}{2} g^{i \tau} g_{\tau \sigma, n},
$$

where we used the fact that in Gaussian normal coordinates, $g_{i n, k}=0, i=$ $1, \cdots, n$. Thus

$$
K_{i \sigma}=-\frac{1}{2} g_{i \sigma, n}
$$

as claimed.

In the Gaussian normal coordinates $w$ associated with a given co-dimension one surface $\Sigma$ and a Lipschitz continuous metric $g=g^{L} \cup g^{R}$, (where we assume as usual that $g^{L}$ and $g^{R}$ are smooth), the metric $g$ is determined on $\Sigma$, but the first derivatives of the metric suffer a jump discontinuity at $\Sigma$. Thus the second fundamental form $K$, which depends on the first derivatives of the ambient metric $g$, also suffers a jump discontinuity at $\Sigma$. In this case it follows from Lemma 2 that $K^{L}$ and $K^{R}$, the second fundamental forms on $\Sigma$ for the metrics $g^{L}$ and $g^{R}$, respectively, are given by (3.25), for $g=g^{L}, g^{R}$, respectively. Thus the following corollary of Israel is immediate.

Corollary 1 The metric components of $g=g^{L} \cup g^{R}$ in Gaussian normal coordinates are $C^{1}$ functions of the coordinate variables if and only if $[K]=$ $\left(K^{R}-K^{L}\right)=0$ at each point on the surface $\Sigma$.

The next lemma expresses the components of the connection coefficients for the ambient metric $g$ in Gaussian normal coordinates in terms of quantities intrinsic to the shock surface. We state this for a smooth metric, and see that it applies to each side $g=g^{L}$ and $g=g^{R}$ separately when the metric is only Lipschitz continuous.

Lemma 4 The components in Gaussian normal coordinates of the connection coefficients for a metric $g$ at a point $P \in \Sigma$ are given by

$$
\begin{gathered}
\Gamma_{i j}^{k}=\tilde{\Gamma}_{i j}^{k}, \quad i, j, k \neq n, \\
\Gamma_{i j}^{n}=K_{i j}, \quad i, j \neq n, \\
\Gamma_{i n}^{k}=-K_{i}^{k}, \quad i, k \neq n,
\end{gathered}
$$




$$
\Gamma_{i n}^{n}=0 .
$$

Here, $\tilde{\Gamma}$ denotes the $(n-1)$-dimensional connection coefficients computed from the intrinsic metric $\tilde{g}$ on $\Sigma$ with $w$-components $\tilde{g_{i j}}, i, j=1, \cdots, n-1$.

Proof: To obtain (3.26), use (2.10) to write

$$
\Gamma_{i j}^{K}=\frac{1}{2} g^{k \sigma}\left\{-g_{i j, \sigma}+g_{\sigma i, j}+g_{j \sigma, i}\right\}
$$

Since $g^{k \sigma}=0$ when $\sigma=n$ and $k \neq n$, it follows that

$$
\Gamma_{i n}^{k}=\tilde{\Gamma_{i n}^{k}}
$$

which is (3.26). Similarly, statement (3.27) follows from

$$
\Gamma_{i j}^{n}=\frac{1}{2} g^{n \sigma}\left\{-g_{i j, \sigma}+g_{\sigma i, j}+g_{j \sigma, i}\right\}
$$

statement (3.28) follows from

$$
\Gamma_{i n}^{k}=\frac{1}{2} g^{k \sigma}\left\{-g_{i n, \sigma}+g_{\sigma i, n}+g_{n \sigma, i}\right\} ;
$$

and statement (3.29) follows from

$$
\Gamma_{i n}^{n}=\frac{1}{2} g^{n \sigma}\left\{-g_{i n, \sigma}+g_{\sigma i, n}+g_{n \sigma, i}\right\},
$$

upon noting that in Gaussian normal coordinates $w$ we have $g^{n \alpha}=0$ unless $\alpha=n$, and $g_{\alpha n, \beta}=0$ for $\alpha, \beta=1, \cdots, n$.

The next lemma uses Lemmas 1 and 4 to expresses the components in Gaussian normal coordinates of the Riemann curvature tensor for the ambient metric $g$ in terms of quantities intrinsic to the shock surface (GaussCodazzi Equations). Again we state this for a smooth metric, and see that it applies to each side $g=g^{L}$ and $g=g^{R}$ separately when the metric is only Lipschitz continuous.

Lemma 5 The components in Gaussian normal coordinates of the Riemann curvature tensor for a metric $g$ at a point $P \in \Sigma$ are given by

$$
R_{j k l}^{i}=\tilde{R}_{j k l}^{i}+K_{l}^{i} K_{j k}-K_{k}^{i} K_{j l}, \quad i, j, k, l \neq n,
$$


which is equivalent to

$$
R_{k l}^{i j}=\tilde{R}_{k l}^{i j}+K_{l}^{i} K_{k}^{j}-K_{k}^{i} K_{l}^{j}, \quad i, j, k, l \neq n
$$

moreover,

$$
R_{i j k}^{n}=K_{i k ; j}-K_{i j ; k}, \quad i, j, k \neq n
$$

where in (3.37), the semicolon denotes covariant differentiation in the surface $\Sigma$. Statement (3.37) is equivalent to

$$
R_{j k}^{n i}=K_{k ; j}^{i}-K_{j ; k}^{i} . \quad i, j, k \neq n
$$

Proof: For (3.35), write

$$
R_{j k l}^{i}=\Gamma_{j l, k}^{i}-\Gamma_{j k, l}^{i}+\Gamma_{\sigma k}^{i} \Gamma_{j l}^{\sigma}-\Gamma_{\sigma l}^{i} \Gamma_{j k}^{\sigma} .
$$

Thus, since only $\sigma$ can be $n$, we have

$$
R_{j k l}^{i}=\tilde{R}_{j k l}^{i}+\Gamma_{n k}^{i} \Gamma_{j l}^{n}-\Gamma_{n l}^{i} \Gamma_{j k}^{n},
$$

which by (3.26) gives (3.35). Statement (3.36) follows because $g^{i n}=0$ for $i \neq n$. For (3.37), write

$$
R_{i j k}^{n}=\Gamma_{i k, j}^{n}-\Gamma_{i j, k}^{n}+\Gamma_{\sigma j}^{n} \Gamma_{i k}^{\sigma}-\Gamma_{\sigma k}^{n} \Gamma_{i j}^{\sigma},
$$

which gives (3.37) on applying (3.27). In this case as before, (3.38) follows from (3.37) because $\tilde{g}^{i n}=g^{i n}$ when $i \neq n$.

The next lemma uses (3.36) and (3.38) to expresses the components in Gaussian normal coordinates of the Einstein curvature tensor for the ambient metric $g$ in terms of quantities intrinsic to the shock surface. Again we state this for a smooth metric, and see that it applies to each side $g=g^{L}$ and $g=g^{R}$ separately when the metric is only Lipschitz continuous, (c.f. [22]).

Lemma 6 The components in Gaussian normal coordinates of the Einstein curvature tensor for a metric $g$ at a point $P \in \Sigma$ are given by

$$
\begin{gathered}
G_{n}^{n}=\frac{1}{2}\left\{(\operatorname{tr} K)^{2}-\operatorname{tr}\left(K^{2}\right)\right\}-\frac{1}{2} \tilde{R}, \\
G_{i}^{n}=-\left\{(\operatorname{tr} K)_{; i}+(\operatorname{div} K)_{i}\right\},
\end{gathered}
$$

where $\tilde{R}$ denotes the curvature scalar for the metric $\tilde{g}$ intrinsic to $\Sigma$, and the semicolon denotes covariant differentiation in the surface $\Sigma$. 
Proof: To prove (3.39), use (3.22) to write

$$
G_{n}^{n}=-\sum_{\sigma, \tau \neq n} R_{[\sigma \tau]}^{[\sigma \tau]}
$$

so that by (3.36)

$$
G_{n}^{n}=-\sum_{\sigma, \tau \neq n} \tilde{R}_{[\sigma \tau]}^{[\sigma \tau]}+\sum_{\sigma, \tau \neq n}\left\{K_{\sigma}^{[\sigma} K_{\tau}^{\tau]}-K_{\tau}^{[\sigma} K_{\sigma}^{\tau]}\right\}
$$

where the sum must be taken over indices $\sigma<\tau$. But by definition,

$$
\tilde{R}=\tilde{R}_{i j}^{i j}=2 \sum_{\sigma, \tau \neq n} \tilde{R}_{[\sigma \tau]}^{[\sigma \tau]},
$$

and

$$
(\operatorname{tr} K)^{2}-\operatorname{tr}\left(K^{2}\right)=\left(K_{i}^{i}\right)^{2}-K_{j}^{i} K_{i}^{j}=2 \sum_{i<j}\left\{K_{i}^{i} K_{j}^{j}-K_{j}^{i} K_{i}^{j}\right\} .
$$

Using these in (3.41) yields (3.39)

To prove (3.40), use (3.23) to write

$$
-G_{i}^{n}=-\sum_{\tau \neq i, n} R_{[i \tau]}^{[n \tau]}=\sum_{j=1}^{n-1} R_{[i j]}^{n j},
$$

where we have applied the antisymmetry of the curvature tensor. Thus by (3.38),

$$
-G_{i}^{n}=\sum_{j=1}^{n-1}\left\{K_{j ; i}^{j}-K_{i ; j}^{j}\right\},
$$

from which (3.40) follows at once. We can now give the

Proof of Theorem 2: Assume that $g=g^{L} \cup g^{R}$, where the metric $g$ is smooth on either side of a co-dimension one shock surface $\Sigma$, and is Lipschitz continuous across the surface. Let $w$ denote the Gaussian normal coordinates associated with the surface $\Sigma$ and the metric $g$. Then we can apply (3.39) and (3.40) of Lemma 6 to $g^{L}$ from the left and $g^{R}$ from the right of $\Sigma$, respectively, to obtain

$\left[G_{n}^{n}\right]=\left[\frac{1}{2}\left\{(\operatorname{tr} K)^{2}-\operatorname{tr}\left(K^{2}\right)\right\}\right]=\frac{1}{2}\left\{\left(\operatorname{tr} K^{R}\right)^{2}-\operatorname{tr}\left(\left(K^{R}\right)^{2}\right)\right\}-\frac{1}{2}\left\{\left(\operatorname{tr} K^{L}\right)^{2}-\operatorname{tr}\left(\left(K^{L}\right)^{2}\right)\right\}$ 
and

$\left[G_{i}^{n}\right]=\left[\left\{-(\operatorname{tr} K)_{, i}+(\operatorname{div} K)_{i}\right\}\right]=\left\{-\left(\operatorname{tr} K^{R}\right)_{, i}+\left(\operatorname{div} K^{R}\right)_{i}\right\}-\left\{-\left(\operatorname{tr} K^{R}\right)_{, i}+\left(\operatorname{div} K^{R}\right)_{i}\right\}$

Here we use the fact that $\tilde{R}$ and $\sum_{j=1}^{n-1}\left\{K_{k i} g^{l j} \tilde{\Gamma}_{l j}^{k}-K_{k j} g^{l j} \tilde{\Gamma}_{l i}^{k}\right\}$ are equal on $\Sigma$ for $g^{L}$ and $g^{R}$ because they depend only on intrinsic properties of the metric $g$ restricted to $\Sigma$, and these agree because of the assumed continuity of $g$. But in Gaussian normal coordinates, $\mathbf{n}=\frac{\partial}{\partial w^{n}}$, and so the jump conditions (3.5) in Gaussian normal coordinates reduce to the condition

$$
\left[G_{\alpha}^{n}\right]=0, \quad \alpha=1, \cdots, n .
$$

Now since $G$ transforms like a tensor under arbitrary $C^{1}$-coordinate transformations, the conditions (3.46) are equivalent to the statement $\left[G_{\beta}^{\alpha}\right] n_{\alpha}=0$ in the original $y$-coordinates. Thus, in light of (3.46), we conclude that (3.11) and (3.12) of Theorem 2 follow directly from (3.44) and (3.45).

In view of Corollary 1 of Lemma 3, we can also conclude the following corollary of Theorem 2, (due to Israel), which gives a global criterion for conservation across $\Sigma,[12,22]$.

Corollary 2 If $[K]=0$ at each point of $\Sigma$, then the jump conditions $\left[G_{\beta}^{\alpha}\right] n_{\alpha}=$ 0 must hold at the point $P$. Moreover, since in this case the metric is $C^{1}$ in Gaussian normal coordinates, the condition $[K]=0$ is also a necessary and sufficient condition for the original Lipschitz continuous metric components $g_{\alpha \beta}$ in the $y$-coordinates to be equivalent to a $C^{1}$ metric under a $C^{1,1}$-transformation of the coordinate variables.

Proof: The sufficiency is clear, and the necessity of this condition follows because, if the metric is equivalent to a $C^{1}$ metric under some regular $C^{1,1}$ coordinate transformation, then the mapping from these coordinates to the Gaussian normal coordinates is a $C^{2}$ mapping, and thus the metric in Gaussian normal coordinates will be $C^{1}$, which implies that the second fundamental form is continuous across the surface. (Note that $[K]=0$ at a point is not sufficient for conservation $\left[G_{j}^{i}\right] \mathbf{n}_{i}=0$ at the point.)

We now show that $R_{i j}$ and $G_{i j}$, viewed as second order operators on the metric components $g_{i j}$, have delta-function singularities at a point $P \in \Sigma$ if 
and only if $[K] \neq 0$ at $P$. Thus, let $g=g^{L} \cup g^{R}$ be Lipschitz continuous across a shock surface $\Sigma$ in $x$-coordinates. The strategy is as follows: we first do the case when $x$ is a Gaussian normal coordinate system defined in a neighborhood of $P \in \Sigma$. We then show that delta-function sources appear at $P \in \Sigma$ in $x$-coordinates if and only if they appear in any coordinate system related to $x$ by a $C^{1,1}$ coordinate transformation. Since any coordinate system in which $g$ is Lipschitz continuous is related to the Gaussian normal coordinates by a $C^{1,1}$ coordinate transformation, it follows that delta-functions appear if and only if $[K] \neq 0$. We then show that when delta-function singularities appear in $G_{i j}$ at $P \in \Sigma$ in a given coordinate system $x$, the metric is not locally Lorentzian at $P$ in sense that there does not exist a $C^{1,1}$ coordinate transformation that takes $x$-coordinates to coordinates in which the metric is locally Lorentzian at $P$, more specifically, such that $g_{i j, k}(P)=0$. Finally, we show, surprisingly, that delta-function singularities never appear in the scalar curvature $R$ at any point on a shock- wave discontinuity on either side of which $g$ is smooth, but across which $g$ is Lipschitz continuous, and this is due to a cancellation of delta-functions in the sum $R_{\sigma}^{\sigma}$.

Lemma 7 Let $x$ be the Gaussian normal coordinates containing a point $P \in$ $\Sigma$, where $\Sigma$ is any smooth surface, so that $\frac{\partial}{\partial n}$ is the normal direction on $\Sigma$. Then the second order $n$-derivatives of $g_{i j}$ that appear in the formula for the Ricci tensor $R_{i j}$, occur only in the terms $R_{i j}, i \neq n, j \neq n$, and in $R_{n n}$, and these are given by

$$
R_{i j}=-\frac{1}{2} g_{i j, n n}+\text { lower order } n-\text { derivatives, } i \neq n, j \neq n
$$

and

$$
R_{n n}=\frac{1}{2} g^{\alpha \beta} g_{\alpha \beta, n n}+\text { lower order } n \text {-derivatives }
$$

where the sum in the last formula is taken over $\alpha, \beta \neq n$.

Proof: From (3.29), assuming Gaussian Normal coordinates, we have

$$
\Gamma_{i n}^{n}=0
$$

Consider $R_{i j}=R_{i \sigma j}^{\sigma}$, which is given by the formulas 


$$
\begin{gathered}
R_{i \beta j}^{\alpha}=\Gamma_{i j, \beta}^{\alpha}-\Gamma_{i \beta, j}^{\alpha}+\Gamma_{\tau \beta}^{\alpha} \Gamma_{i j}^{\tau}-\Gamma_{\tau j}^{\alpha} \Gamma_{i \beta}^{\tau} \\
R_{i j}=R_{i \sigma j}^{\sigma}=\Gamma_{i j, \sigma}^{\sigma}-\Gamma_{i \sigma, j}^{\sigma}+\Gamma_{\tau \sigma}^{\sigma} \Gamma_{i j}^{\tau}-\Gamma_{\tau j}^{\sigma} \Gamma_{i \sigma}^{\tau} .
\end{gathered}
$$

Now since $g$ is Lipschitz continuous across $\Sigma$, and $R_{i j}$ involves second derivatives of $g$, it follows that delta-functions in $R_{i j}$ can arise at $P \in \Sigma$ only in the second order $n$-derivatives appearing in the formula for $R_{i j}$. To see this, note that in Gaussian normal coordinates, $g_{i n}=\delta_{i n}$, and $g_{i j}$ are arbitrary for $i, j=1, \cdots, n-1$. Thus the first derivatives in $k \neq n$ are Lipschitz continuous across $\Sigma$ because $g^{L}=g^{R}$ on $\Sigma$, and thus $g_{i j, k n}$ involves at worst jump discontinuities for $k \neq n$. Now from (3.51), the second order $n$-derivatives can come only from $\Gamma_{i j, \sigma}^{\sigma}$ or $\Gamma_{i \sigma, j}^{\sigma}$. In the former, this can only happen when $\sigma=n$, so consider

$$
\Gamma_{i j, n}^{n}=\frac{1}{2} g^{\sigma n}\left\{-g_{i j, \sigma n}+g_{\sigma i, j n}+g_{j \sigma, i n}\right\} .
$$

But $g^{\sigma n}=0$ unless $\sigma=n$, which implies

$$
\Gamma_{i j, n}^{n}=\frac{1}{2}\left\{-g_{i j, n n}+g_{n i, j n}+g_{j n, i n}\right\} .
$$

Thus we conclude that when $i=n$ or $j=n$, there are no non-zero second order $n$-derivatives in $\Gamma_{i j, n}^{n}$, and when $i, j \neq n, \Gamma_{i j, \sigma}^{\sigma}$ gives rise to only one second order $n$-derivative, namely, $\frac{1}{2} g_{i j, n n}$; i.e.,

$$
\Gamma_{i j, \sigma}^{\sigma}=\frac{1}{2} g_{i j, n n}+\text { lower order } n-\text { derivatives. }
$$

Consider now $\Gamma_{i j, n n}^{\sigma}$, which can have second order $n$-derivatives only for $j=n$ :

$$
\Gamma_{i \sigma, n}^{\sigma}=\frac{1}{2} g^{\sigma \tau}\left\{-g_{i \sigma, \tau n}+g_{\tau i, \sigma n}+g_{\sigma \tau, i n}\right\} .
$$

The first two terms, $g_{i \sigma, \tau n}$ and $g_{\tau i, \sigma n}$, inside the bracket in (3.54), can have second order $n$-derivatives only when $\sigma=n$ or $\tau=n$, in which case $\sigma=n=\tau$ (because $g_{\text {in }}=0$ ), which implies that both of these terms are zero because $\Gamma_{i n}^{n}=0$. But the third term $g_{\sigma \tau, i n}$ in the bracket in (3.54) has second order $n$-derivatives only when $i=n$, and thus we have 


$$
\Gamma_{n \sigma, n}^{\sigma}=\frac{1}{2} g^{\alpha \beta} g_{\alpha \beta, n n}+\text { lower order } n \text {-derivatives, }
$$

and $\Gamma_{i \sigma, j}^{\sigma}$ is a lower order $n$-derivative if $i \neq n$ or $j \neq n$. Thus we conclude that the second order $n$-derivatives in the Ricci tensor occur only in the terms $R_{i j}, i \neq n, j \neq n$, and in $R_{n n}$, and these are given by (3.47) and (3.48).

We now consider the scalar curvature $R$ and the curvature tensors $R_{i j}$ and $G_{i j}$ as second order distribution derivatives of the metric components $g_{i j}$ in Gaussian normal coordinates when $g$ is only Lipschitz continuous on $\Sigma$. In general we expect that second order distribution derivative of $g$ will introduce delta-function singularities on $\Sigma$. The following corollary gives necessary and sufficient conditions for the appearance of such delta-function singularities on $\Sigma$.

Corollary 3 Let $g=g^{L} \cup g^{R}$ be any metric that is Lipschitz continuous across a shock surface $\Sigma$, and smooth on either side of $\Sigma$. Then in Gaussian normal coordinates the scalar curvature $R$, viewed as a second order distribution derivative of the metric components $g_{i j}$, has at worst a jump discontinuity at each $P \in \Sigma$; the Ricci and Einstein curvature tensors $R_{i j}$ and $G_{i j}$ have delta-function singularities at $P \in \Sigma$ if and only if $[K] \neq 0$ at $P$.

Proof: Assuming Gaussian normal coordinates, we have from (3.47) and (3.48) that

$$
R=g^{\sigma \tau} g_{\sigma \tau, n n}-g^{i j} g_{i j, n n}+\text { lower order } n \text {-derivatives. }
$$

and thus the formula for $R$ in terms of $g$ contains no second order $n$-derivatives in Gaussian normal coordinates for any Lipschitz continuous shock-wave, and hence $R$ is at most discontinuous on $\Sigma$. Moreover, in Gaussian normal coordinates $K_{i j}=g_{i j, n}, i, j \neq n$, and hence if $[K] \neq 0$ at $P \in \Sigma$, then $g_{i j, n}$ must suffer a jump discontinuity at $P$ for some $(i, j), i, j \neq n$. Thus by $(3.47), R_{i j}$ is given by the delta function $g_{i j, n n}$ plus a discontinuous function. Conversely, if $[K]=0$ at $P \in \Sigma$, then $g_{i j, n n}$ is at most discontinuous at $P$, and thus $R_{i j}$ is at most discontinuous at $P$. Since $G_{i j}=R_{i j}-\frac{1}{2} R g_{i j}$, and $R$ is at most discontinuous, we conclude that in Gaussian normal coordinates, $R_{i j}$ and $G_{i j}$ contain delta-function singularities if and only if $[K] \neq 0 . \square$.

Now let $\mathcal{R}=R_{j k l}^{i}$ denote the components of the full Riemann curvature tensor in $x$-coordinates, and let $\overline{\mathcal{R}}=\bar{R}_{\beta \gamma \delta}^{\alpha}$ denote the components in a coordinate system $y$ related to $x$ by a $C^{1,1}$ coordinate transformation. Note that 
in any coordinate system, the components of the curvature tensor are given by (3.50), and hence are determined by the same second order differential operator $L$ on the metric components, thus $\mathcal{R}=L[g]$, and $\overline{\mathcal{R}}=L[\bar{g}]$. We note that the highest order derivative terms in $L$ are of the form a function of the unknowns $g_{i j}$ times linear second order differential operators. Thus it is possible to define solutions $g$ that have only weak (distributional) derivatives of second order. The following lemma demonstrates that curvature tensors defined from $L$ in the weak sense continue to transform by the tensor transformation laws under arbitrary $C^{1,1}$ transformations of the coordinates.

Lemma 8 Let $\mathcal{R}$ be a weak solution of $\mathcal{R}=L[g]$ in $x$-coordinates. Then $\overline{\mathcal{R}}=$ $\mathcal{R} \frac{\partial x}{\partial y}$ is a weak solution of $\overline{\mathcal{R}}=L[\bar{g}]$ for any coordinate system $y$ related to $x$ by a $C^{1,1}$ coordinate transformation, where we use the short-hand notation

$$
\mathcal{R} \frac{\partial x}{\partial y}=\mathcal{R}_{j k l}^{i} \frac{\partial x^{j}}{\partial y^{\beta}} \frac{\partial x^{k}}{\partial y^{\gamma}} \frac{\partial x^{l}}{\partial y^{\delta}} \frac{\partial y^{\alpha}}{\partial x^{i}},
$$

and multiplication by a function is taken in the weak sense.

Proof: For smooth $g$ and smooth test functions $\varphi$, let

$$
\int_{\mathbf{R}^{4}} L[g] \varphi=\int_{\mathbf{R}^{4}} L^{*}[g, \varphi]
$$

where $L^{*}[g, \varphi]$ denotes the expression obtained from $L[g]$ by integrating the second order derivatives in $g$ once by parts. Since the second order derivatives in $L$ are given by ${ }^{4}$

$$
\begin{aligned}
R_{j k l}^{i}= & \Gamma_{j l, k}^{i}-\Gamma_{j k, l}^{i}+\text { l.o.t.'s } \\
= & \left(g^{i \sigma}\left\{-g_{j l, \sigma}+g_{\sigma j, l}+g_{l \sigma, j}\right\}\right)_{, k} \\
& -\left(g^{i \sigma}\left\{-g_{j k, \sigma}+g_{\sigma j, l}+g_{k \sigma, j}\right\}\right)_{, l}+\text { l.o.t.'s } \\
= & g^{i \sigma}\left\{-g_{j l, \sigma k}+g_{l \sigma j k}+g_{j k, \sigma l}-g_{k \sigma, j l}\right\}+\text { l.o.t.'s },
\end{aligned}
$$

i.e., are of the form $g^{m n} g_{i j, k l}$, it follows that $L^{*}[g, \varphi]$ contains at worst products of the metric entries $g_{i j}$, the test function $\varphi$, and their first derivatives. Thus

\footnotetext{
${ }^{4}$ Here "l.o.t." denotes "lower order terms", i.e., terms that contain lower order $n$ derivatives.
} 
the integral in the weak formulation $\int_{\mathbf{R}^{4}} L^{*}[g, \varphi]$ is finite for any Lipschitz continuous metric $g$ and any Lipschitz continuous test function $\varphi$ (of compact support).

Now assume that $\mathcal{R}=R_{j k l}^{i}$ is a weak solution of $\mathcal{R}=L[g]$, i.e., $\mathcal{R}$ is a linear functional on the space of Lipschitz continuous test functions, (a distribution), that solves

$$
\langle\mathcal{R}, \varphi\rangle \equiv \int_{\mathbf{R}^{4}} \mathcal{R} \varphi=\int_{\mathbf{R}^{4}} L^{*}[g, \varphi],
$$

for every Lipschitz continuous test function $\varphi$. Note that if $\frac{\partial x}{\partial y}$ is Lipschitz continuous, then the derivatives are bounded, and thus if we let $\bar{g}=g \frac{\partial x}{\partial y}$ be short-hand notation for

$$
\bar{g} \equiv \bar{g}_{\alpha \beta}=g_{i j} \frac{\partial x^{i}}{\partial y^{\alpha}} \frac{\partial x^{j}}{\partial y^{\beta}} \equiv g \frac{\partial x}{\partial y},
$$

then $L^{*}\left[g \frac{\partial x}{\partial y}, \varphi\right]$ is bounded for any Lipschitz continuous test function $\varphi$.

So to prove the lemma, let $g$ be an arbitrary (non-degenerate) Lipschitz continuous metric, let $\varphi$ be an arbitrary Lipschitz continuous test function, and assume that the coordinate systems $x$ and $y$ are related by a $C^{1,1}$ coordinate transformation, (so that in particular, both $\frac{\partial x}{\partial y}$ and $\frac{\partial y}{\partial x}$ are regular, Lipschitz continuous maps). Let $\bar{g}^{\epsilon}$ denote a smooth regularization of the metric $\bar{g}_{\alpha \beta}$, and let $x^{\epsilon}(y)$ denote a regularization of the coordinate map $x(y)$ so that $x^{\epsilon}(y)$ is smooth and has a smooth inverse. We can clearly choose these regularizations so that $\bar{g}_{\alpha \beta}^{\epsilon} \rightarrow \bar{g}_{\alpha \beta}$ in $C^{0,1}, x^{\epsilon}(y) \rightarrow x(y)$ in $C^{1,1}, \frac{\partial x^{\epsilon}}{\partial y}(y) \rightarrow \frac{\partial x}{\partial y}(y)$ in $C^{0,1}$ and $\frac{\partial y}{\partial x^{\epsilon}}\left(x^{\epsilon}\right) \rightarrow \frac{\partial y}{\partial x}(x)$ in $C^{0,1}$. Then

$$
g^{\epsilon} \equiv \bar{g}^{\epsilon} \frac{\partial y}{\partial x^{\epsilon}} \rightarrow g
$$

and

$$
\bar{g}^{\epsilon} \rightarrow \bar{g}
$$

in $C^{0,1}$. Define

$$
\overline{\mathcal{R}}^{\epsilon}=L\left(\bar{g}^{\epsilon}\right),
$$

and

$$
\mathcal{R}^{\epsilon}=\overline{\mathcal{R}}^{\epsilon} \frac{\partial y}{\partial x^{\epsilon}}
$$


Now it follows directly from definitions that

$$
\left\langle\mathcal{R}^{\epsilon} \frac{\partial y}{\partial x^{\epsilon}}, \varphi\right\rangle=\left\langle\overline{\mathcal{R}}^{\epsilon}, \varphi\right\rangle=\int_{\mathbf{R}^{4}} L^{*}\left[g^{\epsilon} \frac{\partial y}{\partial x^{\epsilon}}, \varphi\right] .
$$

But (3.61) simply says that $\mathcal{R}^{\epsilon} \frac{\partial y}{\partial x^{\epsilon}}$ is the curvature tensor for the metric $g^{\epsilon} \frac{\partial y}{\partial x^{\epsilon}}$, and since everything in (3.61) is smooth, we know from the fact that the curvature transforms as a tensor that $\mathcal{R}^{\epsilon}$ must be the curvature tensor for the metric $g^{\epsilon}$; i.e., since everything in (3.61) is smooth, we know that (3.61) holds for every $\varphi \in C^{0,1}$ if and only if

$$
\left\langle\mathcal{R}^{\epsilon}, \varphi\right\rangle=\int_{\mathbf{R}^{4}} L^{*}\left[g^{\epsilon}, \varphi\right]
$$

holds for every $\varphi \in C^{0,1}$. Since $g^{\epsilon} \rightarrow g$ in $C^{0,1}$, (3.62) implies that, as $\epsilon \rightarrow 0$, $\mathcal{R}^{\epsilon}$ tends in the sense of distributions to the distribution $T$, where $T$ satisfies

$$
\langle T, \varphi\rangle=\int_{\mathbf{R}^{4}} L^{*}[g, \varphi]
$$

Therefore (3.63) demonstrates that $T=\mathcal{R}$ as a distribution. Thus, in the limit $\epsilon \rightarrow 0$, we conclude from (3.63) that $\mathcal{R}^{\epsilon} \rightarrow \mathcal{R}$, from (3.61) that $\overline{\mathcal{R}}^{\epsilon} \rightarrow \overline{\mathcal{R}}$, and hence from (3.60) that $\overline{\mathcal{R}}=\mathcal{R} \frac{\partial y}{\partial x}$ in the sense of distributions. This completes the proof of the lemma.

Theorem 3 Assume that $g=g^{L} \cup g^{R}$ is smooth on either side of a 3dimensional shock surface $\Sigma$, and is Lipschitz continuous across $\Sigma$. Then the scalar curvature $R$, when viewed as a second order operator (in the weak sense) on the metric components $g_{i j}$, produces at most a jump discontinuity (i.e., no delta-function singularities) at $P \in \Sigma$; and the curvature tensors $R_{j k l}^{i}, R_{i j}$ and $G_{i j}$ produce no $\delta$-function singularities at $P \in \Sigma$ if and only if the jump in the second fundamental form $K$ satisfies $[K]=0$ at $P$.

Proof: By Corollary 1, the theorem is true in Gaussian normal coordinates $x$, and thus by Corollary 3 and Lemma 8 it holds in any coordinate system $y$ which is $C^{1,1}$ related to $x$. Since for any metric $g=g^{L} \cup g^{R}$ which is smooth on either side of $\Sigma$ and Lipschitz continuous across $\Sigma$, the transformation to Gaussian normal coordinates is an invertible $C^{1,1}$ coordinate transformation, the theorem follows at once. $\square$ 
As a direct corollary of Theorem 3 we see that there exists a locally Lorentzian coordinate frame in a neighborhood of a point $P$ on a Lipschitz continuous shock surface $\Sigma$ if and only if $[K]=0$ at $P$; namely, we have

Corollary 4 Assume that $g=g^{L} \cup g^{R}$ is smooth on either side of a 3dimensional shock surface $\Sigma$, and is Lipschitz continuous across $\Sigma$ in a coordinate system $y$ defined in a neighborhood of $P \in \Sigma$. Then there exists a regular $C^{1,1}$ coordinate transformation $y \rightarrow x$ such that $x$ is locally Lorentzian for $g$ at $P,\left(g_{i j}=\eta_{i j}\right.$ and $g_{i j, k}=0$ at $\left.P\right)$, if and only if $[K]=0$ at $P$.

Proof: Assume $[K]=0$ at $P$, and choose locally Lorentzian coordinates at $P$ for the smooth metric obtained by restricting $g$ to the surface $\Sigma$ in a neighorhood of $P$ in the surface $\Sigma$. Extend these coordinates to Gaussian normal coordinates $x$ based on these surface coordinates, the $x$ coordinates being defined in an $n$-dimensional neighborhood of $P$. Then in $x$-coordinates the metric components $g_{i j}$ satisfy $g_{i j}=\eta_{i j}$ and $K_{i j}=g_{i j, n}=0$ at $P$, and so $x$ is locally Lorentzian at $P$. Conversely, assume that $[K] \neq 0$, but that there exists a coordinate transformation $y \rightarrow x$ such that, in $x$-coordinates, $g_{i j}=\eta_{i j}$ and $g_{i j, k}=0$ at $P$. Then in $x$-coordinates, $g$ is $C^{1}$ at $P$, and hence there are no delta-function singularities in the components $g_{i j}$ of $g$ in $x$ coordinates. Thus by Theorem $3,[K]=0$, and hence the locally Lorentzian coordinates $x$ cannot exist when $[K] \neq 0$.

The next result partially validates the statement that real shock-waves cannot form in solutions of the source free Einstein equations $R_{i j}=0$, or equivalently $G_{i j}=0$, by demonstrating that "shock-waves" in solution metrics are only coordinate anomalies in the sense that they can be transformed away by coordinate transformation. Note that the theorem allows for the possibility that dicontinuities can form in solutions, (which we expect because the equations are nonlinear quasilinear in nature), but asserts that if the solution metric is Lipschitz continuous across a smooth surface, but smooth on either side, then there is a coordinate transformation such that in the new coordinates, the metric is smooth across the surface.

Corollary 5 Assume that the components of $g=g^{L} \cup g^{R}$ in a coordinate system y are Lipschitz continuous across a smooth 3-dimensional shock surface $\Sigma$, are $C^{k}$ functions of $y$ on either side of $\Sigma$, and assume that all $k$ derivatives are continuous up to the boundary $\Sigma$ from either side of $\Sigma$. Assume also that $g$ is a weak solution of $R_{\alpha \beta}=0$ or $G_{\alpha \beta}=0$ when viewed 
as second order operators on the metric components $g_{\alpha \beta}$. Then in Gaussian normal coordinates $x$, (which are $C^{1,1}$ related to the original coordinates), the metric components $g_{i j}$ are actually $C^{k}$ functions of $x$ across $\Sigma$.

Proof: Assume first that $g=g^{L} \cup g^{R}$ is a weak solution of $R_{\alpha \beta}=0$. But $R_{i j}=0$ in the weak sense across $\Sigma$ implies that there are no $\delta$-function sources in $R_{i j}$ on $\Sigma$, and thus by the previous theorem, $[K]=0$ across $\Sigma$. Thus Israel's result implies that $g_{i j, k}$ are all continuous across $\Sigma$, and since $G_{i j} \equiv 0$, the jump conditions are automatically satisfied across $\Sigma$. It follows from (3.47) and (3.48) that in Gaussian normal coordinates,

$$
R_{i j}=-\frac{1}{2} g_{i j, n n}+\text { lower order } n-\text { derivatives, } i \neq n, j \neq n,
$$

and

$$
R_{n n}=\frac{1}{2} g^{i j} g_{i j, n n}+\text { lower order } n-\text { derivatives } .
$$

But since the $g_{i j, k}$ are continuous across $\Sigma$, it follows that the lower order terms in (3.64) and (3.65) must be continuous functions across $\Sigma$, our assumptions implying that the derivatives of $g$ in the surface $\Sigma$ are the same for $g^{L}$ and $g^{R}$. But since $R_{i j}=0$ for both $g^{L}$ and $g^{R}$, we can solve for $g_{i j, n n}$ in (3.64) and (3.65) in terms of lower order derivatives that are continuous across $\Sigma$, and conclude that $g_{i j, n n}$ must also be continuous across $\Sigma$ for all $i, j=1, \cdots, n$. (Recall that $g_{n i}=$ constant in Gaussian normal coordinates.) This shows us that $k^{\prime}$ th order derivatives of $g_{i j}$ which are up to second order in $x^{n}$, are in fact continuous functions of $x$ across $\Sigma$ in Gaussian normal coordinates. Now differentiate (3.64) and (3.65) with respect to $x^{n}$. Then the differentiated lower order terms in (3.64) and (3.65) are continuous across $\Sigma$, and hence again we can solve for $g_{i j, n n n}$ in terms of functions that are continuous across $\Sigma$. Thus we conclude that $k$ 'th order derivatives of $g_{i j}$ which are up to second order in $x^{n}$, are in fact continuous functions of $x$ across $\Sigma$ in Gaussian normal coordinates $x$. Continuing, we see that all the $k^{\prime}$ th order derivatives of $g_{i j}$ are continuous across $\Sigma$ in Gaussian normal coordinates. Since, by Corollary 3 , the scalar curvature never contains delta-function singularities on $\Sigma$, the result for $R_{i j}$ implies the same result for $G_{i j}$.

The same argument establishes the following more general version of this corollary: 
Corollary 6 Assume that $g=g^{L} \cup g^{R}$ is smooth on either side of a 3dimensional shock surface $\Sigma$, and is Lipschitz continuous across $\Sigma$ in some coordinate system $y$. Assume that $g$ is a weak solution of $G_{\alpha \beta}=\kappa T_{\alpha \beta}$ that contains no delta-function singularities on $\Sigma$. Then in Gaussian normal coordinates the metric components $g_{i j}$ are $C^{2}$ functions of $x$ if and only if $[G]=0$ across $\Sigma$.

Summary: The results of this section are summarized in the following theorem:

Theorem 4 Let $\Sigma$ denote a smooth, 3-dimensional shock surface in spacetime with spacelike normal vector $\mathbf{n}$. Assume that the components $g_{i j}$ of the gravitational metric $g$ are smooth on either side of $\Sigma$, (continuous up to the boundary on either side separately), and Lipschitz continuous across $\Sigma$ in some fixed coordinate system. Then the following statements are equivalent:

(i) $[K]=0$ at each point of $\Sigma$.

(ii) The curvature tensors $R_{j k l}^{i}$ and $G_{i j}$, viewed as second order operators on the metric components $g_{i j}$, produce no delta function sources on $\Sigma$.

(iii) For each point $P \in \Sigma$ there exists a $C^{1,1}$ coordinate transformation defined in a neighborhood of $P$, such that, in the new coordinates, (which can be taken to be the Gaussian normal coordinates for the surface), the metric components are $C^{1,1}$ functions of these coordinates.

(iv) For each $P \in \Sigma$, there exists a coordinate frame that is locally Lorentzian at $P$, and can be reached within the class of $C^{1,1}$ coordinate transformations.

Moreover, if any one of these equivalencies hold, then the Rankine-Hugoniot jump conditions, $[G]_{i}^{\sigma} n_{\sigma}=0$, (which express the weak form of conservation of energy and momentum across $\Sigma$ when $G=\kappa T$ ), hold at each point on $\Sigma$.

Here $[K]$ denotes the jump in the second fundamental form (extrinsic curvature) $K$ across $\Sigma$, (this being determined by the metric separately on each side of $\Sigma$ because $g_{i j}$ is only Lipschitz continuous across $\Sigma$ ), and by $C^{1,1}$ we mean that the first derivatives are Lipschitz continuous. Theorem 4 should be credited mostly to Israel, [12], who obtained results (i)-(iii) in Gaussian normal coordinates. Our contribution was to identify the covariance class of $C^{1,1}$ transformations, and to thereby obtain precise coordinate independent statements for (ii) and (iii), as well as the equivalence with (iv). As a consequence of this, we obtain the result that the Ricci scalar curvature $R$ never 
has delta function sources at a Lipschitz continuous matching of the metrics, as well as the results in Corollarys 5 and 6 which validate the statement that shock-wave singularities in the source free Einstein equations $R_{i j}=0$ or $G_{i j}=0$ can only appear as coordinate anomalies, and can be transformed away by coordinate transformation. Note that when there are delta function sources in $G$ on a surface $\Sigma$, the surface should be interpreted as a surface layer (because $G=\kappa T$ ), and not a true fluid dynamical shock-wave, $[12,22]$. In Theorem 5 below, we show that for spherically symmetric solutions, $[G]_{\sigma \tau} n^{\sigma} n^{\tau}=0$ alone implies the absence of surface layers, (and hence the other equivalencies in Theorem 4), so long as the areas of the spheres of symmetry match smoothly at $\Sigma$. We use this result in our construction of the shock-waves that extend the Oppenheimer-Snyder model to the case of non-zero pressure. The following counter-example shows that in general, the above equivalences can fail even when $\left[G_{i}^{\sigma}\right] n_{\sigma}=0$ holds at each point on $\Sigma .^{5}$

For the counter-example it suffices to show that there exist Lipschitz continuous shock-waves which satisfy the Israel jump relations (3.11) and (3.12) across a shock-wave interface, but which cannot be transformed to a metric that is $C^{1}$ in a neighborhood of each point on the shock. By Corollary 1, it suffices to construct a shock-wave interface across which the Israel conditions are satisfied, but such that the second fundamental form $K$ is not continuous across the interface. To this end, let $g_{i j}$ denote the coordinates of a metric in Gaussian normal coordinates, such that the spacelike normal to the shock surface is given by $\mathbf{n}=\partial / \partial x^{n}$, and $g_{i j}$ is of the form

$$
g_{i j}=\left[\begin{array}{cc}
h_{i j} & 0 \\
0 & 1
\end{array}\right] .
$$

Assume now that the $h_{i j}$ are given by

$$
h_{i j}=\left\{\begin{array}{lll}
\eta_{i j}+a_{i j} x^{n} & \text { if } & x^{n}>0 \\
\eta_{i j}+b_{i j} x^{n} & \text { if } & x^{n}<0
\end{array}\right\}
$$

where $a_{i j}$ and $b_{i j}$ are constants to be determined. Thus by Lemma 3 , the second fundamental forms $K^{L}$ and $K^{R}$ on the left and right of the shock surface are given by $K_{i j}^{L}=a_{i j}$ and $K_{i j}^{R}=b_{i j}, i, j=1, \cdots, n-1$. Since $K_{i j}^{L}$

\footnotetext{
${ }^{5}$ See [12] where such an example is given in which $G \equiv 0$ on both sides of $\Sigma$.
} 
and $K_{i j}^{R}$ are constant,

$$
K_{i, \sigma}^{\sigma}=(\operatorname{tr} K)_{, i}=0,
$$

for $K=K^{L}, K^{R}$. Thus the Israel jump conditions (3.11) and (3.12) reduce to

$$
\left[(\operatorname{tr} K)^{2}-\operatorname{tr}\left(K^{2}\right)\right]=0 .
$$

Hence to satisfy the Israel jump conditions it suffices to find $\mathbf{a} \equiv a_{i j}$ and $\mathbf{b} \equiv b_{i j}$ satisfying

$$
(\operatorname{tr} \mathbf{a})^{2}-\operatorname{tr}\left(\mathbf{a}^{2}\right)=0=(\operatorname{tr} \mathbf{b})^{2}-\operatorname{tr}\left(\mathbf{b}^{2}\right) .
$$

But in the simplest case where $\mathbf{a}$ and $\mathbf{b}$ are $2 \times 2$ matrices,

$$
\operatorname{tr} \mathbf{a}=a_{11}+a_{22},
$$

and

$$
\operatorname{tr}\left(\mathbf{a}^{2}\right)=a_{11}^{2}+2 a_{21} a_{12}+a_{22}^{2}
$$

and so

$$
(\operatorname{tr} \mathbf{a})^{2}-\operatorname{tr}\left(\mathbf{a}^{2}\right)=2 \operatorname{det}(\mathbf{a}) .
$$

Thus we can satisfy the Israel jump conditions by choosing $\mathbf{a}$ and $\mathbf{b}$ to be any $2 \times 2$ matrices with zero determinant. If in addition $a_{i j} \neq b_{i j}$, then $[K]=K^{R}-K^{L} \neq 0$, and so by Theorem 4 , conservation $\left[G_{i}^{n}\right]=0$ holds across the interface $x^{n}=0$, but, in view of Corollary 1 , the metric cannot be transformed to a metric that is globally $C^{1}$ across the shock.

In this section we restrict to spherically symmetric metrics. The theorem to follow states that in the special case of spherical symmetry, the jump conditions $\left[G^{i j}\right] n_{i} n_{j}=0$ that express the weak form of conservation across a shock surface, actually are implied by a single condition, so long as the shock is non-null, and the areas of the spheres of symmetry match smoothly at the shock and change monotonically as the shock evolves. Note that in general, assuming that the angular variables are identified across the shock, we expect conservation to entail two condtions, one for the time and one for the radial components. Thus the fact that the smooth matching of the spheres of symmetry reduces conservation to one conditions can be interpreted as an instance of the general principle that smoothness in the metric implies conservation of the sources. 
Theorem 5 Assume that $g$ and $\bar{g}$ are two spherically symmetric metrics that match Lipschitz continuously across a three dimensional shock interface $\Sigma$ to form the matched metric $g \cup \bar{g}$. That is, assume that $g$ and $\bar{g}$ are Lorentzian metrics given by

$$
d s^{2}=-a(t, r) d t^{2}+b(t, r) d r^{2}+c(t, r) d \Omega^{2},
$$

and

$$
d \bar{s}^{2}=-\bar{a}(\bar{t}, \bar{r}) d \bar{t}^{2}+\bar{b}(\bar{t}, \bar{r}) d \bar{r}^{2}+\bar{c}(\bar{t}, \bar{r}) d \Omega^{2},
$$

and that there exists a smooth coordinate transformation $\Psi:(t, r) \rightarrow(\bar{t}, \bar{r})$, defined in a neighborhood of a shock surface $\Sigma$ given by $r=r(t)$, such that the metrics agree on $\Sigma$. (We implicitly assume that $\theta$ and $\varphi$ are continuous across the surface.) Assume that

$$
c(t, r)=\bar{c}(\Psi(t, r)),
$$

in an open neighborhood of the shock surface $\Sigma$, so that, in particular, the areas of the 2-spheres of symmetry in the barred and unbarred metrics agree on the shock surface. Assume also that the shock surface $r=r(t)$ in unbarred coordinates is mapped to the surface $\bar{r}=\bar{r}(\bar{t})$ by $(\bar{t}, \bar{r}(\bar{t}))=\Psi(t, r(t))$. Assume, finally, that the normal $\mathbf{n}$ to $\Sigma$ is non-null, and that

$$
\mathbf{n}(c) \neq 0
$$

where $\mathbf{n}(c)$ denotes the derivative of the function $c$ in the direction of the vector $\mathbf{n}{ }^{6}$ Then the following are equivalent to the statement that the components of the metric $g \cup \bar{g}$ in any Gaussian normal coordinate system are $C^{1,1}$ functions of these coordinates across the surface $\Sigma$ :

$$
\begin{gathered}
{\left[G_{j}^{i}\right] n_{i}=0,} \\
{\left[G^{i j}\right] n_{i} n_{j}=0,}
\end{gathered}
$$

${ }^{6}$ I.e., we assume that the areas of the 2 -spheres of symmetry change monotonically in the direction normal to the surface. E.g., if $c=r^{2}$, then $\frac{\partial}{\partial t} c=0$, so the assumption $\mathbf{n}(c) \neq 0$ is valid except when $\mathbf{n}=\frac{\partial}{\partial t}$, in which case the rays of the shock surface would be spacelike. Thus the shock speed would be faster than the speed of light rays if our assumption $\mathbf{n}(c) \neq 0$ failed in the case $c=r^{2}$. 


$$
[K]=0 .
$$

Here, $[f]=\bar{f}-f$ denotes the jump in the quantity $f$ across $\Sigma$, and $K$ is the second fundamental form on the shock interface defined by (3.6). ${ }^{7}$

Proof: Let $\left(w^{1}, w^{2}, w^{3}\right)=\left(z^{1}, \theta, \varphi\right)$ be a smooth coordinate system on $\Sigma$, and let $z=\left(z^{0}, \cdots, z^{3}\right)$ denote the extension of these coordinates to a Gaussian normal coordinate system in a neighborhood of $\Sigma$, (where we let $\frac{\partial}{\partial z^{0}} \equiv \frac{\partial}{\partial z^{n}}$ when we restrict to spacetime, c.f. [29]). Then by Lemma $2, \mathbf{n}=\frac{\partial}{\partial z^{0}}$, and $\mathbf{T}=\frac{\partial}{\partial z^{1}}$ is tangent to the shock surface. Now in light of Corollary 2 of Theorem 2 it suffices to verify that (3.6) implies (3.7). By Theorem 2, in $w$-coordinates we have

$$
\left[G^{i j}\right] n_{i} n_{j}=\left[G^{00}\right]=\left[\operatorname{tr}\left(K^{2}\right)-(\operatorname{tr} K)^{2}\right] .
$$

But in Gaussian normal coordinates the metric $g \cup \bar{g}$ is diagonal on the surface $\Sigma$. To see this, note that the restriction of the metric $(g \cup \bar{g})$ to the surface $\Sigma$ is diagonal because the off diagonal $\varphi$ and $\theta$ components are zero in both (3.1) and (3.2), and the metric components $(g \cup \bar{g})_{0 j}$, for $j \neq 0$, are zero throughout any Gaussian normal coordinate frame in a whole neighborhood of $\Sigma$. Thus, by Lemma 3,

$$
K_{i j}=-\frac{1}{2} g_{i j, 0} .
$$

Therefore, since $g \cup \bar{g}$ is diagonal on $\Sigma, K$ is also diagonal, and so the only non-zero components of $K$ are

$$
\begin{aligned}
& K_{11}=-\frac{1}{2} g_{11,0}, \\
& K_{22}=-\frac{1}{2} g_{22,0},
\end{aligned}
$$

and

$$
K_{33}=-\frac{1}{2} g_{33,0} .
$$

\footnotetext{
${ }^{7}$ This does not contradict the spherical shell example of Israel in [12] because (3.3) fails in that example.
} 
But, since $c$ and $\bar{c}$, (defined in (3.1) and (3.2)), transform like functions under arbitrary $(t, r)$-transformations, (3.3) implies that $c$ and $\bar{c}$ define the same invariant function in a neighborhood of $\Sigma$. Thus, by (3.3) and the fact that $c=g_{22}=\bar{c}=\bar{g}_{22}$ on $\Sigma$, we see that $g_{22,0}=\mathbf{n}(c)=\bar{g}_{22,0} \neq 0$ and $g_{33,0}=\mathbf{n}(c) \sin ^{2} \theta=\bar{g}_{33,0} \neq 0$ on the surface $\Sigma$, and hence

$$
\left[K_{22}\right]=0
$$

and

$$
\left[K_{33}\right]=0
$$

across $\Sigma$. Now we have

$$
0=\left[G_{i j}\right] n_{i} n_{j}=\left[G_{00}\right]=\left[\operatorname{tr}\left(K^{2}\right)-(\operatorname{tr} K)^{2}\right]=-2\left[K_{11}\right]\left(K_{22}+K_{33}\right),
$$

and since $\left(K_{22}+K_{33}\right) \neq 0$, (by the assumption $\mathbf{n}(c) \neq 0$ ), we conclude that (3.6) and (3.15) imply

$$
\left[K_{11}\right]=0 .
$$

Since $K_{i j}$ is diagonal, (3.13), (3.14) and (3.16) imply (3.7), so (3.6) implies (3.7), and we are done.

\section{Matching an FRW to a TOV Metric Across a Shock-Wave}

In this section apply the theory of Section 3 to the general problem of matching a Friedmann-Robertson-Walker metric (FRW) to a Tolmann-OppenheimerVolkoff (TOV) metric Lipschitz continuously across a radial shock-wave. We will first show that given any such metrics, one can always in principle construct a coordinate mapping between the FRW and the TOV coordinates such that, under this coordinate identification, the FRW and TOV metrics match Lipshitz continuously across an interface that is implicitly determined. In order for the matching to describe a true shock-wave, the further constraint of conservation must be imposed, and this restricts the possible FRW and TOV metrics that can be matched across a shock-wave. An application of 
Theorem 5 demonstrates that the conservation constraint reduces to a single condition, and this allows for the possibility of nontrivial examples. (The constraint can be viewed as a restriction on the possible equations of state on either side of the shock). The conservation constraint is shown to reduce to a quartic equation in the densities and pressures on either side of the shock. An application of MAPLE shows that the quartic factors, and we use this to show that two possible types of pressure jumps are allowed, and one of them can be ruled out by physical considerations. We use this formulation of the conservation constraint in the next section to construct a simple class of exact FRW-TOV shock-wave solutions under the assumption that the FRW and TOV equations of state are each of the form $p=\sigma \rho$, where $\sigma$ is constant. (That is, in these examples, we assume that the sound speed $\sqrt{\sigma}$ is (a different) constant on either side of the shock-wave.

\subsection{The General FRW-TOV Matching Problem}

The FRW metric describes a spherically symmetric spacetime that is homogeneous and maximally symmetric at each fixed time, [42]. In coordinates, the FRW metric is given by,

$$
d s^{2}=-d t^{2}+R^{2}(t)\left\{\frac{1}{1-k r^{2}} d r^{2}+r^{2} d \Omega^{2}\right\},
$$

where $t \equiv x^{0}, r \equiv x^{1}, \theta \equiv x^{2}, \varphi \equiv x^{3}, R \equiv R(t)$ is the 'cosmological scale factor', and $d \Omega^{2}=d \theta^{2}+\sin ^{2} \theta d \varphi^{2}$ denotes the standard metric on the unit 2 -sphere. The constant $k$ can be normalized to be either $+1,-1$, or 0 by appropriately rescaling the radial variable, and each of the three cases is qualitatively different. This induces a rescaling of $R(t)$, and so alternatively, $R(t)$ can be rescaled to any positive value at a fixed time, (say $R=1$ at present time), in which case only the sign of $k$ is unchanged. The sign of $k$ gives the sign of the curvature in the constant curvature surfaces at each fixed $t$, and so from (4.1) it is clear that the 3 -space at $t=$ const is unbounded when $k \leq 0$, and when $k>0, r=1 / \sqrt{k}$ marks the outer boundary of the coordinate system in (4.1). In standard theory of cosmology, the $k=0$ case corresponds to critical expansion, $k>0$ to a closed universe, and $k<0$ to an open universe. Current estimates of the Hubble constant $H=\dot{R} / R$ argue for an open universe. 
To obtain the equation for $R(t)$ implied by the Einstein equations, assume that the stress energy tensor is of the form

$$
T^{i j}=\bar{p} g^{i j}+(\bar{p}+\bar{\rho}) u^{i} u^{j},
$$

for a perfect fluid, and that the fluid is co-moving with the metric, [42]. The fluid is said to be co-moving relative to a background metric $g_{i j}$ if $u^{i}=0$, $i=1,2,3$, so that $g$ diagonal and $g_{i j} u^{i} u^{j}=-1$ imply that

$$
u^{0}=\sqrt{-g_{00}} .
$$

Substituting (4.1) into the Einstein equations (2.16), and making the assumption that the fluid is perfect and co-moving with the metric, yields the following constraints on the unknown functions $R(t), \rho(t)$ and $p(t),[42,30]$ :

$$
\begin{gathered}
3 \ddot{R}=-4 \pi \mathcal{G}(\rho+3 p) R, \\
R \ddot{R}+2 \dot{R}^{2}+2 k=4 \pi \mathcal{G}(\rho-p) R^{2},
\end{gathered}
$$

together with

$$
\dot{p} R^{3}=\frac{d}{d t}\left\{R^{3}(p+\rho)\right\} .
$$

Equation (4.6) is equivalent to

$$
p=-\rho-\frac{R \dot{\rho}}{3 \dot{R}} .
$$

Substituting (4.4) into (4.5) we get

$$
\dot{R}^{2}+k=\frac{8 \pi \mathcal{G}}{3} \rho R^{2} .
$$

Since $\rho$ and $p$ are assumed to be functions of $t$ alone in (4.1), equations (4.7) and (4.8) give two equations for the two unknowns $R$ and $\rho$ under the assumption that the equation of state is of the form $p=p(\rho)$. It follows from (4.7)-(4.8), c.f., [30], that $(R(t), \rho(t))$ is a solution if and only if $(R(-t), \rho(-t))$ is a solution, and that

$$
\dot{\rho} \dot{R}<0 \text {. }
$$


Thus to every expanding solution there exists a corresponding contracting solution, and conversely.

The TOV metric describes a time-independent, spherically symmetric solution that models the interior of a star. In coordinates the components of the metric are given by

$$
d \bar{s}^{2}=-B(\bar{r}) d \bar{t}^{2}+A(\bar{r})^{-1} d \bar{r}^{2}+\bar{r}^{2} d \Omega^{2} .
$$

We write this metric in bar-coordinates so that it can be distinguished from the unbarred coordinates when the metrics are matched. When $M(\bar{r}) \equiv$ $M_{0} \equiv$ const, and $B=A_{-1}$ the metric reduces to the (empty space) Schwarzschild metric, and the singularity at $\bar{r}=2 \mathcal{G} M_{0}$ is referred to as the Schwarzschild radius for the mass $M_{0}$, and represents the edge of a black hole. (See [33, 34] for proof that Black Holes cannot form in smooth TOV metrics that solve the Einstien equations with nonzero sources.) Assuming the stress tensor is that of a perfect fluid which is co-moving with the metric, and substituting (4.10) into the field equations (2.16), yields, (c.f. [42]),

$$
A(\bar{r})=\left(1-\frac{2 \mathcal{G} M}{\bar{r}}\right)
$$

where $M \equiv M(\bar{r}), \bar{\rho} \equiv \bar{\rho}(\bar{r})$ and $\bar{p} \equiv \bar{p}(\bar{r})$ satisfy the following system of ordinary differential equations in the unknown functions $(\bar{\rho}(\bar{r}), p(\bar{r}), M(\bar{r}))$ :

$$
\begin{gathered}
\frac{d M}{d \bar{r}}=4 \pi \bar{r}^{2} \bar{\rho} \\
-\bar{r}^{2} \frac{d \bar{p}}{d \bar{r}}=\mathcal{G} M \bar{\rho}\left\{1+\frac{\bar{p}}{\bar{\rho}}\right\}\left\{1+\frac{4 \pi \bar{r}^{3} \bar{p}}{M}\right\}\left\{1-\frac{2 \mathcal{G} M}{\bar{r}}\right\}^{-1} .
\end{gathered}
$$

Equation (4.13) is called the Oppenheimer-Volkov equation, and is referred to by Weinberg as the fundamental equation of Newtonian astrophysics. ([42], page 301).

In this section we assume the case of a barotropic equation of state $\bar{p}=$ $\bar{p}(\bar{\rho})$, in which case equations (4.12), (4.13) yield a system of two ODE's in the two unknowns $(\bar{\rho}, M)$. We always assume that

$$
0<\frac{\bar{p}}{\bar{\rho}} \equiv \bar{\mu}<1
$$

and that the sound speed is less than the speed of light $c=1$, 


$$
0<\bar{\sigma} \equiv \frac{d \bar{p}}{d \bar{\rho}} \leq 1
$$

The total mass $M$ inside radius $\bar{r}$ is then defined by

$$
M(\bar{r})=\int_{0}^{\bar{r}} 4 \pi \xi^{2} \bar{\rho}(\xi) d \xi .
$$

The metric component $B \equiv B(\bar{r})$ is determined from $\bar{\rho}$ and $M$ through the equation

$$
\frac{B^{\prime}(\bar{r})}{B}=-2 \frac{\bar{p}^{\prime}(\bar{r})}{\bar{p}+\bar{\rho}}
$$

In the special case when the density $\bar{\rho}$ is assumed to be constant, one can solve the Oppenheimer-Volkoff equations for the pressure, and the resulting solution, first discussed by Schwarzschild, is referred to as the Interior Schwarzschild metric.

We remark that for any given FRW and TOV metrics, there are maximal domains of definitions for the variables. We assume that the FRW metric is defined on the maximal interval $t_{-}<t<t_{+}$and $0 \leq r_{-}<r<r_{+}$, and the TOV metric is defined on the maximal interval $0<\bar{r}_{-}<\bar{r}<\bar{r}_{+}$. For example, if $k>0$, then we must have $r<\frac{1}{\sqrt{k}}, t$ must be restricted so that $\rho(t)$ and $R(t)$ are positive, and by (4.8), we must require $\frac{8 \pi \mathcal{G}}{3} \rho(t) R(t)^{2}-k \geq 0$.

We now construct a coordinate mapping $(t, r) \rightarrow(\bar{t}, \bar{r})$, such that, under this coordinate identification, the FRW metric (4.1) matches the TOV metric (4.10) Lipschitz continuously across an interface $r=r(t)$ that arises implicitly from the matching procedure. That is, we define a coordinate mapping that takes the unbarred frame of the FRW metric over to a barred TOV coordinate system that leaves fixed the $\theta$ and $\varphi$ coordinates. In order to apply Theorem 5 of Section 3, we require that the areas of the 2-spheres of symmetry of the FRW (4.1) metric agree with the areas of the 2-spheres of symmetry of the TOV metric (4.10). Thus to start, assume that

$$
\bar{r}^{2} d \Omega^{2}=R^{2} r^{2} d \Omega^{2}
$$

so that

$$
\bar{r}=R r .
$$


That is, we define the first component of the coordinate mapping $(t, r) \rightarrow$ $(\bar{t}, \bar{r})$ by

$$
\bar{r}=\bar{r}(t, r)=R(t) r .
$$

Note that at this stage the transformation $\bar{r}=R r$ is defined globally, which is important in order to appy Theorem 5 of Section 3, which requires that (4.16) hold not just at the shock surface, but in an open neighborhood of the shock surface.

We next use (4.16) to rewrite the FRW metric in $(t, \bar{r})$-coordinates. We have from (4.16) that

$$
d \bar{r}=R d r+\dot{R} r d t
$$

SO

$$
d r=\frac{1}{R} d \bar{r}-\frac{\dot{R}}{R} r d t
$$

and thus

$$
d r^{2}=\frac{1}{R^{2}} d \bar{r}^{2}+\frac{\dot{R}^{2}}{R^{2}} r^{2} d t^{2}-2 \frac{\dot{R}}{R^{2}} \bar{r} d t d \bar{r}
$$

Thus, the FRW metric (4.1) is given in $(t, \bar{r})$-coordinates by

$$
d s^{2}=-\left\{1-\frac{\dot{R}^{2} \bar{r}^{2}}{R^{2}-k \bar{r}^{2}}\right\} d t^{2}+\frac{R^{2}}{R^{2}-k \bar{r}^{2}} d \bar{r}^{2}-\frac{2 R \dot{R} \bar{r}}{R^{2}-k \bar{r}^{2}} d t d \bar{r}+\bar{r}^{2} d \Omega^{2}
$$

which, using

$$
\left\{R^{2}-k \bar{r}^{2}-\dot{R}^{2} \bar{r}^{2}\right\}=R^{2}\left\{1-\frac{8 \pi \mathcal{G}}{3} \rho R^{2} r^{2}\right\}
$$

becomes

$$
d s^{2}=\frac{1}{R^{2}-k \bar{r}^{2}}\left\{-R^{2}\left(1-\frac{8 \pi \mathcal{G}}{3} \rho R^{2} r^{2}\right) d t^{2}+R^{2} d \bar{r}^{2}-2 R \dot{R} \bar{r} d t d \bar{r}\right\}+\bar{r}^{2} d \Omega^{2}
$$


We can now complete the definition of the coordinate identification $(t, r) \rightarrow$ $(\bar{t}, \bar{r})$ by defining $\bar{t}=\bar{t}(t, r)$ so as to eliminate the cross term $d t d \bar{r}$ in (4.22). We do this first for a general metric of the form

$$
d \tilde{s}^{2}=-C(t, \bar{r}) d t^{2}+D(t, \bar{r}) d \bar{r}^{2}+2 E(t, \bar{r}) d t d \bar{r} .
$$

It is not hard to verify that if $\psi=\psi(t, \bar{r})$ is chosen to satisfy the equation

$$
\frac{\partial}{\partial \bar{r}}(\psi C)=-\frac{\partial}{\partial t}(\psi E)
$$

then

$$
d \bar{t}=\psi(t, \bar{r})\{C(t, \bar{r}) d t-E(t, \bar{r}) d \bar{r}\},
$$

is an exact differential. Since (4.25) defines the coordinate $\bar{t}$ as a function of $(t, \bar{r})$, and we already have $\bar{r}=R(t) r$, it follows that (4.24) defines $\bar{t}=\bar{t}(t, r)$, thus completing the definition of the sought after coordinate transformation $(t, r) \rightarrow(\bar{t}, \bar{r})$. Assuming (4.25), the $(\bar{t}, \bar{r})$ line element for (4.23) becomes

$$
d \tilde{s}^{2}=-\left(\psi^{-2} C^{-1}\right) d \vec{t}^{2}+\left(D+\frac{E^{2}}{C}\right) d \bar{r}^{2} .
$$

Now in terms of the metric

$$
d \tilde{s}^{2}=-R^{2}\left[1-\frac{8 \pi \mathcal{G}}{3} \rho R^{2} r^{2}\right] d t^{2}+R^{2} d \bar{r}^{2}-2 R \dot{R} \bar{r} d \bar{t} d \bar{r},
$$

which appears in $(4.22), C, D$ and $E$ are given by

$$
\begin{gathered}
C=R^{2}\left\{1-\frac{8 \pi \mathcal{G}}{3} \rho R^{2} r^{2}\right\}, \\
D=R^{2},
\end{gathered}
$$

and

$$
E=-R \dot{R} \bar{r}
$$

Thus, using (4.27), the FRW metric in $(\bar{t}, \bar{r})$-coordinates becomes

$$
d s^{2}=\frac{1}{R^{2}-k \bar{r}^{2}}\left\{-\left(\psi^{2} C\right)^{-1} d \bar{t}^{2}+\left(D+\frac{E^{2}}{C}\right) d \bar{r}^{2}\right\}+\bar{r}^{2} d \Omega^{2} .
$$


But from (4.28)-(4.30) we obtain

$$
D+\frac{E^{2}}{C}=R^{2}+\frac{R^{2} \dot{R}^{2} \bar{r}^{2}}{R^{2}\left(1-\frac{8 \pi \mathcal{G}}{3} \rho \bar{r}^{2}\right)}=R^{2}+\frac{\dot{R}^{2} R^{2} r^{2}}{1-\frac{8 \pi \mathcal{G}}{3} \rho R^{2} r^{2}}
$$

Now equating the $d \bar{r}^{2}$ coefficients in the TOV solution (4.10) and the FRW solution (4.31) and using (4.32), we obtain the equation for the shock surface: ${ }^{8}$

$$
\left(R^{2}-k \bar{r}^{2}\right)\left(1-\frac{2 \mathcal{G} M}{\bar{r}}\right)^{-1}=R^{2}+\frac{\dot{R}^{2} R^{2} r^{2}}{1-\frac{8 \pi \mathcal{G}}{3} \rho R^{2} r^{2}},
$$

which, using (4.8) simplifies to

$$
M(\bar{r})=\frac{4 \pi}{3} \rho(t) \bar{r}^{3} .
$$

Hence (4.34) defines the shock surface, and the shock surface in $(t, r)$-coordinates can be obtained from (4.34) by making the substitution $\bar{r}=R(t) r$. (Of course, additional assumptions are required to insure that the shock surface as defined implicitly by (4.34) is reasonable, for example, stays within the domain of definition of the FRW metric, namely, $1-k r^{2}>0$, when $k>0$, etc.) It remains only to determine $\psi$ from (4.24) so that the $d \bar{t}^{2}$ terms in the TOV and FRW metric agree on this surface. To obtain $\psi$, which determines the coordinate $\bar{t}$ in terms of the $(t, r)$ coordinates of the FRW metric in a neighborhood of the shock surface, we solve the equation (4.24) subject to initial data on the shock surface which is forced upon us by the condition that the $d \bar{t}^{2}$ terms match on the shock surface. So, equating the $d \bar{t}^{2}$ terms in (4.10) and (4.31), our assumption is that

$$
\frac{1}{R^{2}-k \bar{r}^{2}} \frac{1}{\psi^{2} C}=B(\bar{r})
$$

holds on the shock surface (4.34). Rewrite (4.24) in the form of a first-order linear partial differential equation for $\psi$,

$$
C \psi_{\bar{r}}+E \psi_{t}=f(t, \bar{r}, \psi)
$$

\footnotetext{
${ }^{8}$ Note that, interestingly, the $d \bar{r}^{2}$ coefficients are independent of $\psi$.
} 
Here, $C$ and $E$ are functions of $t$ and $\bar{r}$ given by (4.28) and (4.30), and thus we can solve the initial value problem $(4.36)$ in $(t, \bar{r})$-coordinates with initial data (4.35) given on the shock surface (4.34), provided that the shock surface is non-characteristic for (4.36).

Now the characteristics for (4.36) are given by

$$
\lambda \equiv \frac{d \bar{r}}{d t}=\frac{C}{E},
$$

so that the function $\psi$ is obtained by solving the ODE

$$
\frac{d \psi}{d \mu}=f(t, \bar{r}, \psi)
$$

starting with initial values on the shock surface (4.34), where $\frac{d}{d \mu}$ denotes differentiation in the $(E, C)$-direction in $(t, \bar{r})$-coordinates. Solving (4.35) for $\psi$ gives the initial values of $\psi$ to be met on the shock surface; namely,

$$
\psi^{2}=\frac{1}{B\left(R^{2}-k \bar{r}^{2}\right) C} .
$$

Thus, if $\frac{d \bar{r}}{d t}$ denotes the speed of the shock surface in $(t, \bar{r})$-coordinates, then the condition that the shock surface be non-characteristic at a point is, by (4.37), that

$$
\frac{d \bar{r}}{d t} \neq \frac{C}{E}
$$

If (4.40) holds at a point on the shock surface (4.34), then we can solve (4.36) uniquely for $\psi$ in a neighborhood of the point, thereby matching the FRW and TOV solutions in a Lipschitz continuous manner in a neighborhood of such a point on the surface in the $(\bar{t}, \bar{r})$-coordinate system. Since we need only define local coordinate systems in order to define a spacetime manifold, the shock surface (4.34) defines a complete Lipschitz matching of the metrics FRW and TOV at each point of the surface where the non-characteristic condition (4.40) holds. It is interesting to observe that one need not explicitly solve the PDE (4.36) for $\psi$ in order to determine the shock surface equation (4.34), and the solution of (4.34) can be calculated even when we do not have a closed form expression for $\bar{t}$ as a function of $t$ and $r$. That is, we find it somewhat remarkable that, other than it's existence, we do not require any 
detailed information about the transformation $\bar{t}=\bar{t}(t, r)$ in the subsequent developments.

We shall discuss the condition (4.40) further below in Propositions 2 and 3, but first we discuss the equation for the shock surface (4.34). This is necessary in order to obtain an expression for the shock speed, and to motivate the conditions in Propositions 2 and 3 below. Note first that we have not made any choice regarding whether the FRW metric is on the "inside" or the "outside" of the TOV solution. For the case of a star, the FRW metric is on the inside (at small values of $\bar{r}$ within the shock surface), and the TOV is on the outside of the shock surface. For definiteness, we will only consider this case, although the discussion we give below applies equally well to the case when the FRW metric is on the outside.

The shock position is defined implicitly by (4.34). Note that (4.34) allows an interpretation of a global principle of conservation of mass in the special coordinate $\bar{r}$. Indeed, $M\left(\bar{r}_{0}\right)$ is the total mass that would appear inside the radius $\bar{r}_{0}$ were the Tolman-Oppenheimer-Volkoff solution continued to values of $\bar{r}<\bar{r}_{0}$. Thus, $M(\bar{r})$ represents the total mass that is generating the TOV solution outside the radius $\bar{r}=\bar{r}_{0}$. This describes the left-hand-side of (4.34). The right-hand-side of (4.34) can be interpreted as the total mass inside the sphere of radius $\bar{r}_{0}$ at a fixed time $t$ in the Freidmann-Robertson-Walker solution. That is, if we interpret $4 \pi \rho R(t)^{3} r_{0}^{3}$ as the total mass behind the shock at fixed $t$ in the FRW metric, then (4.34) says that this is equal to the mass $M\left(r_{0}\right)$ observed by the TOV metric outside the shock, when the shock is at position $\bar{r}_{0}=R(t) r_{0}$. Thus (4.34) says that the "total mass" is conserved as the shock propagates outward. Therefore, the total mass in the TOV solution that an observer sees out at infinity is fixed, and this equals the total mass in the inside FRW metric plus the total mass in the outside TOV metric. As an application of this global conservation of mass principle, we note that since in a "physically relevant" model for a star, the density $\bar{\rho}(\bar{r})$ for the TOV metric should be a decreasing function of $\bar{r}$, the global conservation principle cannot hold when $\bar{\rho}-\rho \equiv[\rho]=0$ across the shock surface. Indeed, if $\frac{d \bar{\rho}}{d \bar{r}}<0$ for $\bar{r}<\bar{r}_{0}$, and $\rho\left(t_{0}\right)=\bar{\rho}\left(\bar{r}_{0}\right)$, then

$$
\frac{4 \pi}{3} \rho\left(t_{0}\right) \bar{r}^{3}=\frac{4 \pi}{3} \bar{\rho}\left(\bar{r}_{0}\right) \bar{r}^{3}<\int_{0}^{\bar{r}_{0}} 4 \pi \bar{\rho}(\xi) \xi^{2} d \xi=M\left(\bar{r}_{0}\right),
$$

and so by $(4.34)$, the point $\left(t_{0}, \bar{r}_{0}\right)$ cannot lie on the shock surface: the global conservation of mass principle implies that if $\frac{d \bar{\rho}}{d \bar{r}}<0$, then $[\rho] \neq 0$ across the 
shock.

With this motivation, we can now calculate the shock speed under the condition $[\rho] \neq 0$. Indeed, by the implicit function theorem, the shock surface (4.34) is given by $\bar{r}=\bar{r}(t)$ provided that

$$
\frac{d M}{d \bar{r}}-4 \pi \rho(t) \bar{r}^{2} \neq 0
$$

But, using (4.12), (4.42) becomes

$$
4 \pi \bar{r}^{2}(\bar{\rho}-\rho) \neq 0
$$

at a point on the shock surface. Thus, as we have shown above, if we assume that $\frac{d \bar{\rho}}{d \bar{r}}<0$, this condition is always valid on the shock surface. We can now calculate the speed of the shock $s \equiv \dot{\bar{r}}$, (where "dot" denotes $\frac{d}{d t}$.) Using (4.34), which we write in the form

$$
M(\bar{r}(t))=\frac{4 \pi}{3} \rho(t) \bar{r}(t)^{3},
$$

and differentiating with respect to $t$, we find

$$
s \equiv \dot{\bar{r}}=\frac{\dot{\rho} \bar{r}}{3[\rho]} .
$$

Since $[\rho]<0$, (we are assuming that $\frac{d \bar{\rho}}{d \bar{r}}<0$ ), the shock speed is negative if $\dot{\rho}>0$ and is positive if $\dot{\rho}<0$. Observe that, from (4.37), the condition on the shock speed (4.45) that guarantees that the surface be non-characteristic at a point is, (c.f. (4.39),

$$
\left(\frac{\dot{\rho} \bar{r}}{3[\rho]}\right)^{2} \neq \frac{C^{2}}{E^{2}}=\frac{\left(\frac{8 \pi \mathcal{G}}{3} \rho \bar{r}^{2}-1\right)^{2}}{\frac{8 \pi \mathcal{G}}{3} \rho \bar{r}^{2}-k r^{2}},
$$

where we have used (4.8), (4.16), (4.28), (4.30). Note that in the classical theory of shock-waves, the stable shock-waves always advance toward the side of the shock where the fluid pressure is lower, and the corresponding shock-waves that move into the higher pressure side are unstable, and are referred to as rarefaction shocks, [27]. This means that if $\frac{d \bar{\rho}}{d \bar{r}}>0$, then the shock is stable if $s>0,(\dot{\rho}<0)$, and unstable if $s<0,(\dot{\rho}>0)$. We remark that all of the above development is independent of the equations of state $p=p(\rho)$ and $\bar{p}=\bar{p}(\bar{\rho})$. The famous example of Oppenheimer and 
Snyder [25], is obtained in the limit when the pressure $p \equiv 0$, and the TOV solution is replaced by the Schwarzschild metric, (4.10) assuming a constant mass function $M(\bar{r}) \equiv M=$ const, $B=A^{-1}$. In this case the FRW solution satisfies $\rho(t) R(t)^{3}=\rho(0)$, and so for a particular solution satisfying $R(0)=1$, $\dot{R}(0)=0,(4.8)$ implies that $k=\frac{8 \pi \mathcal{G}}{3}$. Thus (4.34) reproduces the well known result that the radius of the star $a$ at time $t=0$ in the Oppenheimer-Snyder model is given by the relation, (see [42], page 346),

$$
M=\frac{4 \pi}{3} \rho(0) a^{3} .
$$

Note that in the Oppenheimer-Snyder limit, the interface must be interpreted as a contact discontinuity rather than a shock-wave because $a \equiv$ const and thus no energy or momentum is transported across the interface.

The following proposition gives identities that hold at the shock surface as a consequence of (4.34) and the coordinate identification $(t, r) \rightarrow(\bar{t}, \bar{r})$. These will be useful in later developments.

Proposition 1 On the shock surface (4.34), the following identities hold

$$
\begin{gathered}
\frac{1}{\psi^{2} C^{2}}=B\left(1+\frac{A E^{2}}{C^{2}}\right)=\frac{B}{A}\left(1-k r^{2}\right), \\
C=R^{2} A \\
\frac{E}{C}=\frac{-\dot{R} r}{A} \\
\frac{E^{2}}{C^{2}}=\frac{-A+\left(1-k r^{2}\right)}{A^{2}} \\
\dot{R}^{2} r^{2}=-A+\left(1-k r^{2}\right),
\end{gathered}
$$

Proof: The transformation $\Psi$ that maps the $(t, r)$-coordinates of the FRW metric to the $(\bar{t}, \bar{r})$-coordinates of the TOV metric is given by

$$
\begin{gathered}
d \bar{r}=\dot{R} r d t+R d r, \\
d \bar{t}=\psi C d t-\psi E d \bar{r}=(\psi C-\psi E \dot{R} r) d t-\psi E R d r,
\end{gathered}
$$


where we have used (4.25) together with the fact that $\bar{r}=\Psi_{2}(t, r)=R(t) r$. From these it follows that

$$
\frac{\partial \overline{\mathbf{x}}^{i}}{\partial \mathbf{x}^{j}}=\left[\begin{array}{cc}
\psi C-\psi E \dot{R} r & -\psi E R \\
\dot{R} r & R
\end{array}\right]_{j}^{i},
$$

where in this section we use the notation $\mathbf{x}=(t, r), \overline{\mathbf{x}}=(\bar{t}, \bar{r})$, and $\tilde{\mathbf{x}}=(t, \bar{r})$, and we supress the $(\theta, \varphi)$ coordinates. (Here, the upper $i$ lower $j$ on the right hand side of (4.52) denotes the $(i, j)$-entry of the matrix.) From these relations it follows easily that

$$
\frac{\partial \tilde{\mathbf{x}}^{i}}{\partial \mathbf{x}^{j}}=\left[\begin{array}{cc}
1 & 0 \\
\dot{R} r & R
\end{array}\right]_{j}^{i}
$$

and

$$
\frac{\partial \tilde{\mathbf{x}}^{i}}{\partial \overline{\mathbf{x}}^{j}}=\left[\begin{array}{cc}
\frac{1}{\psi C} & \frac{E}{C} \\
0 & 1
\end{array}\right]_{j}^{i} .
$$

Now in the tr-coordinate plane, the FRW and TOV metrics have components $g_{R W}^{i j}$ and $g_{I S}^{i j}$ in $\mathbf{x}$ - and $\overline{\mathbf{x}}$-coordinates given respectively by

$$
g_{R W}^{i j}=\left[\begin{array}{cc}
-1 & 0 \\
0 & \frac{1-k r^{2}}{R^{2}}
\end{array}\right]^{i j},
$$

and

$$
g_{I S}^{i j}=\left[\begin{array}{cc}
-B^{-1} & 0 \\
0 & A
\end{array}\right]^{i j},
$$

where $A=1-\frac{2 \mathcal{G} M}{\bar{r}}, B$ satisfies (4.15), and the upper $i j$ denotes the $(i, j)$ entry of the matrix. Now on the shock surface $M=\frac{4 \pi}{3} \rho \bar{r}^{3}$, the metrics $g_{R W}$ and $g_{I S}$ agree, by which we mean that

$$
g_{R W}^{\alpha \beta}=\frac{\partial \mathbf{x}^{\alpha}}{\partial \overline{\mathbf{x}}^{i}} g_{I S}^{i j} \frac{\partial \mathbf{x}^{\beta}}{\partial \overline{\mathbf{x}}^{j}} .
$$

Rather than calculate this out directly, we use the fact that the FRW and TOV metrics must have components that agree on the shock surface in the $\tilde{x}$-coordinates. Thus we calculate 


$$
\tilde{g}_{R W}^{\alpha \beta}=\frac{\partial \tilde{\mathbf{x}}^{\alpha}}{\partial \mathbf{x}^{i}} g_{R W}^{i j} \frac{\partial \tilde{\mathbf{x}}^{\beta}}{\partial \mathbf{x}^{j}}=\left[\begin{array}{cc}
-1 & -\dot{R} r \\
-\dot{R} r & -\dot{R}^{2} r^{2}+\left(1-k r^{2}\right)
\end{array}\right]^{\alpha \beta}
$$

and

$$
\tilde{g}_{I S}^{\alpha \beta}=\frac{\partial \tilde{\mathbf{x}}^{\alpha}}{\partial \overline{\mathbf{x}}^{i}} g_{I S}^{i j} \frac{\partial \tilde{\mathbf{x}}^{\beta}}{\partial \overline{\mathbf{x}}^{j}}=\left[\begin{array}{cc}
-\frac{1}{\psi^{2} C^{2} B}+\frac{A E^{2}}{C^{2}} & \frac{A E}{C} \\
\frac{A E}{C} & A
\end{array}\right]^{\alpha \beta} .
$$

(Again, the superscript $\alpha \beta$ on the RHS of (4.57) and (4.58) denotes the $(\alpha, \beta)$ entry of the matrix.) Equating the $(0,1)$-entries in (4.57) and (4.58) we obtain (4.49). Equating the (1,1)-entries in (4.57) and (4.58) we obtain (4.51), and this together with (4.49) gives (4.50). Equating the (0,0)-entries in (4.57) and (4.58) gives the first equality in (4.47), and applying (4.51) gives the second. Finally, (4.48) follows from (4.49) together with (4.30), $E=-R \dot{R} r$. This concludes the proof of Proposition 1

Alternatively, we can derive (4.47)-(4.51) directly from (4.11),(4.8) and (4.34), together with the expressions (4.28),(4.30) and (4.35) for $C, E$, and $B$, respectively. To obtain (4.51), solve (4.34) for $\rho$, solve (4.11) for $M$, and substitute these into (4.8). To obtain (4.48), multiply (4.8) by $r^{2}$, solve for $\frac{8 \pi \mathcal{G}}{3} \rho R^{2} R^{2}$, and substitute this into (4.28). Using (4.48) together with (4.30), gives (4.49). The identity (4.49) together with (4.51), yields (4.50). Statement (4.35) together with (4.48) gives $\frac{1}{\psi^{2} C^{2}}=\frac{B}{A}\left(1-k r^{2}\right)$. Using (4.48) together with (4.30) and (4.51), in the expression $1+\frac{A E^{2}}{C^{2}}$ gives the last equality in (4.47).

We end this section by giving conditions under which the shock surface is non-characteristic; i.e., that (4.39) holds. We assume here that the shock surface lies within the domain of definition of the FRW metric if $k>0$. The first proposition gives conditions on the equation of state $\bar{p}(\bar{\rho})$ that guarantees the shock surface (4.34) is non-characteristic provided it does not intersect the Schwarzschild radius, $A=1-\frac{2 \mathcal{G} M}{\bar{r}}=0$, of the TOV solution.

Proposition 2 If the equation of state $\bar{p}(\bar{\rho})$ satisfies the condition

$$
\frac{d \bar{p}}{d \bar{\rho}} \geq 0
$$

and

$$
A \neq 0
$$


everywhere on the shock surface (4.34), then the shock surface is nowhere characteristic.

Proof: We already have that (c.f., (4.37), (4.30),)

$$
\lambda=\frac{C}{E}=\frac{C}{R(-\dot{R}) \bar{r}},
$$

and

$$
s=\frac{\dot{\rho} \bar{r}}{3[\rho]} .
$$

From the Oppenheimer-Volkoff equation (4.13) for $\frac{d \bar{p}}{d \bar{r}}$, we see that the sign of $\frac{d \bar{p}}{d \bar{r}}$ is positive inside the Schwarzschild radius and negative outside. Thus $\operatorname{sign}([\rho])=\operatorname{sign}\left(\frac{d \bar{\rho}}{d \bar{r}}\right)=\operatorname{sign}\left(\frac{d \bar{p}}{d \bar{r}}\right)=-\operatorname{sign}(A)$. But on the shock surface, we also have by (4.48)

$$
C=R^{2} A,
$$

and so $\operatorname{sign}(A)=\operatorname{sign}(C)$. Finally, we also have from (4.9) that $\dot{R} \dot{\rho}<0$. Thus,

$$
\operatorname{sign}(\lambda)-\operatorname{sign}(s)=\operatorname{sign}(C)-\operatorname{sign}([\rho])=-\left\{\operatorname{sign}\left(\frac{d \bar{p}}{d \bar{r}}+\operatorname{sign}([\rho])\right\} \neq 0 . \square\right.
$$

We shall also need the following proposition:

Proposition 3 If $\dot{R}=0$ and $A \neq 0$ at a point on the shock surface (4.34), (i.e., the point is not on the Schwarzschild radius), then, if the shock speed is finite at the point, the shock surface is also non-characteristic at the point.

Proof: By (4.36), the characteristic surfaces satisfy

$$
\begin{gathered}
\frac{d \bar{r}}{d s}=C=R^{2} A, \\
\frac{d t}{d s}=E=-R^{3} \dot{R},
\end{gathered}
$$


where we have used (4.48) and (4.49). Therefore, if $\dot{R}=0$, the characteristic is tangent to $t=$ constant, and thus any finite speed $s=\frac{d \bar{r}}{d t}$ is a noncharacteristic speed.

Summary: The results of this section can be summarized as follows: Let (4.1) and (4.10) denote arbitrary FRW and TOV metrics that solve the Einstein equations for a perfect fluid. (We make no restriction on the equation of state at this point.) Then we have identified the following conditions under which there exists a smooth regular coordinate transformation

$$
\Psi:(t, r) \rightarrow(\bar{t}, \bar{r})
$$

and a corresponding shock surface $r=r(t)$ in FRW $(t, r)$-coordinates, (which maps to the curve $\bar{r}=\bar{r}(\bar{t})$ in TOV barred coordinates by $(\bar{t}, \bar{r}(\bar{t}))=\Psi(t, r(t)))$, such that, when written in the same coordinates, the metrics (4.1) and (4.10) agree and are Lipschitz continuous across the shock surface which is given implicitly by the equation $M=4 \pi \rho \bar{r}^{3}$. For example, the metrics agree on the shock surface when both are written in either the barred or unbarred coordinates. We summarize most of the results of this section in the following theorem:

Theorem 6 Assume that the shock surface $\bar{r}=\bar{r}(t)$ is defined implicitly by

$$
M(\bar{r})=\frac{4 \pi}{3} \rho \bar{r}^{3}
$$

in a neighborhood of a point $\left(t_{0}, \bar{r}_{0}\right)$ that satisfies (4.62). Assume that

$$
\bar{r}=\Psi_{2}(t, r)=R(t) r,
$$

so that the sphere's of symmetry agree in the barred and unbarred frames, and the shock surface in $(t, r)$-coordinates is given by $r(t)=\bar{r}(t) / R(t)$. Assume finally that both

$$
\begin{gathered}
1-k r(t)^{2}>0, \\
A\left(\bar{r}_{0}\right) \neq 0,
\end{gathered}
$$

and that the non-characteristic condition 


$$
\frac{d \bar{r}}{d t} \neq \frac{C}{E}=-\frac{\dot{R} r}{A}
$$

hold at $t=t_{0}$, (c.f., (4.28), (4.30) and 4.49). Then the coordinate $\bar{t}=\Psi_{1}(t, r)$ can be defined smoothly and in such a way that $\Psi=\left(\Psi_{1}, \Psi_{2}\right)$ is 1-1 and regular in a neighborhood of the point $\left(t_{0}, r_{0}\right)$, (c.f. (4.36)), and the metrics (4.1) and (4.10) will match in a Lipschitz continuous fashion across the shock surface $r=r(t)$ in a neighborhood of $\left(t_{0}, r_{0}\right)$.

By the implicit function theorem, a sufficient condition for (4.34) to define a surface locally through $\left(t_{0}, \bar{r}_{0}\right)$ is that

$$
\frac{\partial}{\partial \bar{r}} \frac{M}{\bar{r}^{3}}=M-\frac{4 \pi}{3} \bar{\rho} \neq 0 .
$$

By differentiating (4.34) directly, we obtain the alternative sufficient condition,

$$
[\rho] \neq 0 \text {. }
$$

\subsection{The Conservation Constraint}

Assume for this section that we are given smooth FRW (4.1) and a TOV (4.10) solutions of the Einstein equations (2.16) such that Theorem 6 and (4.62)-(4.65) hold for all $t \in\left(t_{-}, t_{+}\right), \bar{r} \in\left(\bar{r}_{-}, \bar{r}_{+}\right)$, and $r=\frac{\bar{r}(t)}{R(t)} \in\left(r_{-}, r_{+}\right)$. That is, assume that the shock surface $\bar{r}=\bar{r}(t)$ is defined by (4.34) and that the metrics agree on this surface throughout this range of variables, when the unbarred coordinates (4.1) and barred coordinates (4.10) are identified by the transformation $(\bar{t}, \bar{r}) \rightarrow(t, r)$ constructed in the last section. Thus $\bar{r}=\bar{r}(t, r)$ is given by

$$
\bar{r}=R(t) r
$$

and the transformation $\bar{t}=\bar{t}(t, r)$ is assumed to exist throughout this interval in light of the non-characteristic assumption (4.65). Other than it's existence, we do not require any detailed information about the $\bar{t}$ transformation in the subsequent development. The following theorem gives conditions under which the matched FRW and TOV metrics define a true shock-wave

solotion of the Einstein equatons: that is, a weak solution such that all of the equivalencies (i)-(iv) of Theorem 4, section 3 are true, and conservation of energy and momentum hold at the interface. 
Theorem 7 Let $g \cup \bar{g}$ denote a metric obtained by matching an FRW metric $g$ and a TOV metric $\bar{g}$ Lipschitz continuously across the interface defined implicitly by (4.34), such that Theorem 6 holds. Assume that at each point of the interface the condition

$$
\left[T^{i j}\right] n_{i} n_{j}=(\bar{p}+\rho) \dot{r}^{2}-(\bar{\rho}+\bar{p}) \frac{\left(1-k r^{2}\right)}{A R^{2}} \dot{\bar{r}}^{2}+(p-\bar{p}) \frac{1-k r^{2}}{R^{2}}=0
$$

where $\dot{r}, \dot{\bar{r}}$ denote the shock speeds $\frac{d r}{d t}, \frac{d \bar{r}}{d t}$, respectively, differentiation being taken with respect to the unbarred FRW time coordinate $t$; and [.] denotes the FRW-TOV jump in a quantity across the interface, as calculated in the same coordinate system. Then the resulting metric $g \cup \bar{g}$ defines a true shock-wave solution of the Einstein equations in the sense that all of the equivalencies of Theorem 4 hold, and these imply that the Rankine-Hugoniot jump conditions

$$
\left[T_{i j}\right] n^{i}=0, \quad i=0, \ldots, 3
$$

hold at the shock.

Proof: Because $\bar{r}=R(t) r$ holds in a neighborhood of the shock surface, conditions (3.3) and (3.4) of Theorem 5 is met. Thus, according to Theorem 5 , all of the equivalencies of Theorem 4 follow from the single (invariant) condition

$$
\left[G_{i j}\right] n^{i} n^{j}=0
$$

which is equivalent to

$$
\left[T_{i j}\right] n^{i} n^{j}=0,
$$

in light of the fact that both the FRW and TOV metric are assumed to satisfy the field equations $G_{i j}=\kappa T_{i j}$ on either side of the shock. We emphasize that the indices $i, j$ must refer to components in the same coordinate system, where coordinates on either side of the shock are identified through the coordinate transformation $(t, r) \rightarrow(\bar{t}, \bar{r})$. To start, use the Einstein equation $G=\kappa T$, the condition $\left[G^{i j}\right] n_{i} n_{j}=0$ for conservation across the shock (c.f.Theorem 5), and the assumption that the source fluid is co-moving with respect to the metrics on either side of the shock (c.f. (4.2)), to rewrite the condition for conservation as 


$$
\left[T^{i j}\right] n_{i} n_{j}=(p-\bar{p})|\mathbf{n}|^{2}+(\rho+p) n_{0}^{2}-(\bar{\rho}+\bar{p}) \frac{\bar{n}_{0}^{2}}{B}=0 .
$$

Here $n_{i}$ and $\bar{n}_{i}$ denote the $i$-components of the normal vector $\mathbf{n}$ to the shock surface (4.34) in unbarred (FRW) and barred (TOV) coordinates, respectively, and $|\mathbf{n}|^{2}=g^{i j} n_{i} n_{j}$. (Note that $u^{i}=\delta_{0}^{i}$ in (FRW) coordinates, $u^{i}=B^{-1 / 2} \delta_{0}^{i}$ in (TOV) coordinates, thus giving rise to the factor $B$.) Since $n_{i}=0=\bar{n}_{i}, i=2,3$, we need only pay attention to the 0 - and 1 -components of $\mathbf{n}$. To verify (4.72), note that, for example, in the (FRW) unbarred frame, (4.2) gives

$$
T^{i j} n_{i} n_{j}=p g^{i j} n_{i} n_{j}+(p+\rho)\left(u^{i} n_{i}\right)^{2}=p|\mathbf{n}|^{2}+(p+\rho)\left(n_{0}\right)^{2} .
$$

Moreover, we need not choose the vector $\mathbf{n}$ to be of unit length, so long as $n_{i}$ and $\bar{n}_{i}$ are the components of the same vector. Since the LHS of (4.72) is an invariant scalar, so is the RHS. In order to evaluate $n_{i}$ and $\bar{n}_{i}$, let (4.34) (formally) define the surface $r=r(t)$, which we can write as the level curve of the scalar $\varphi(t, r)=r-r(t)=0$. Then we can choose $n_{i} d x^{i}=d \varphi$, so that

$$
d \varphi=n_{0} d t+n_{1} d r=-\dot{r} d t+d r
$$

which yields

$$
n_{0}=-\dot{r}
$$

and

$$
n_{1}=1 \text {. }
$$

To obtain $\bar{n}_{i}$, we write the function $\varphi$ in $(\bar{t}, \bar{r})$-coordinates:

$$
\varphi(\bar{t}, \bar{r})=\frac{\bar{r}}{R(t(\bar{t}, \bar{r}))}-r(t(\bar{t}, \bar{r})) .
$$

Then

$$
d \varphi(\bar{t}, \bar{r})=\left\{-\frac{\bar{r}}{R^{2}} \dot{R} \frac{\partial t}{\partial \bar{t}}-\dot{r} \frac{\partial t}{\partial \bar{t}}\right\} d \bar{t}+\bar{n}_{1} d \bar{r}=-\frac{\dot{\bar{r}}}{R} \frac{\partial t}{\partial \bar{t}} d \bar{t}+\bar{n}_{1} d \bar{r}
$$

so that 


$$
\bar{n}_{0}=-\frac{\dot{\bar{r}}}{R} \frac{\partial t}{\partial \bar{t}}
$$

But using the fact that

$$
\bar{r}=R r
$$

together with (4.25),

$$
d \bar{t}=\psi(t, \bar{r})\{C(t, \bar{r}) d t-E(t, \bar{r}) d \bar{r}\}
$$

we have

$$
d t=(\psi C)^{-1} d \bar{t}+\frac{E}{C} d \bar{r}
$$

which implies

$$
\frac{\partial t}{\partial \bar{t}}=(\psi C)^{-1}
$$

Putting this into (4.77) yields

$$
\bar{n}_{0}=-\frac{\dot{\bar{r}}}{R \psi C}
$$

Using the identity (4.47) of Proposition 1 we obtain

$$
\bar{n}_{0}^{2}=\frac{B}{A}\left(1-k r^{2}\right) \bar{r}^{2}
$$

where $-B$ and $A^{-1}=\left(1-\frac{2 \mathcal{G} M}{\bar{r}}\right)^{-1}$ are the coefficients of $d t^{2}$ and $d \bar{r}^{2}$ in the TOV metric (4.10). Finally, using the FRW metric (5.57) to compute $|\mathbf{n}|^{2}$, we obtain

$$
|\mathbf{n}|^{2}=-n_{0}^{2}+\frac{1-k r^{2}}{R^{2}} n_{1}^{2}=-\dot{r}^{2}+\frac{1-k r^{2}}{R^{2}} .
$$

Now substituting (4.73), (4.79) and (4.80) into (4.72) yields

$$
\left[T^{i j}\right] n_{i} n_{j}=(\bar{p}+\rho) \dot{r}^{2}-(\bar{\rho}+\bar{p}) \frac{\left(1-k r^{2}\right)}{A R^{2}} \dot{\bar{r}}^{2}+(p-\bar{p}) \frac{1-k r^{2}}{R^{2}}=0
$$


which is equation (4.69).

Equation (4.69) gives the additional contraint imposed by conservation across the shock in terms of the quantities $r=r(t)$, (the shock position), and the values that $\rho, p, \bar{\rho}, \bar{p}$ and $R$ taken on the shock surface. The following Proposition explains why the pressure must be taken to be zero in the Oppenheimer-Snyder model:

Lemma 9 If $\bar{\rho}=\bar{p}=0$ identically, (so that the TOV solution reduces to the Schwarzschild solution), and $\rho \geq 0$ and $p \geq 0$ everywhere, then (4.69) implies $p=0$ and $r(t)=$ const. all along the shock.

Proof: When $\bar{\rho}=\bar{p}=0$, (4.69) reduces to

$$
\rho \dot{r}^{2}+p \frac{1-k r^{2}}{R^{2}}=0 .
$$

Since $\frac{1-k r^{2}}{R^{2}}>0$ in the FRW metric, the lemma follows at once.

We now derive an equivalent formulation of the conservation constraint (4.69).

Lemma 10 The conservation constraint (4.69) has the equivalent formulation

$$
0=(1-\theta)(\rho+\bar{p})(p+\bar{\rho})^{2}+\left(1-\frac{1}{\theta}\right)(\bar{\rho}+\bar{p})(\rho+p)^{2}+(p-\bar{p})(\rho-\bar{\rho})^{2},
$$

where

$$
\theta=\frac{A}{1-k r^{2}} .
$$

Before giving a proof of (4.82), we first note that, assuming (4.63) and (4.64) hold, the condition $0<\theta \leq 1$ is equivalent to the condition

$$
\dot{R}^{2}=\frac{8 \pi \mathcal{G}}{3} \rho R^{2}-k \geq 0 ;
$$

that is, equivalent to the condition that the shock surface lies within the coordinate restriction of the FRW metric. To see this, use the shock surface equation $M=4 \pi \rho \bar{r}^{3}$ to simplify (4.84) as follows:

$$
\begin{aligned}
\dot{R}^{2} & =\frac{8 \pi \mathcal{G}}{3} \rho R^{2}-k \\
& =\frac{1}{r^{2}}\left\{\frac{2 \mathcal{G}}{\bar{r}}-k r^{2}\right\},
\end{aligned}
$$


and so

$$
\dot{R}^{2} r^{2}=-A+\left(1-k r^{2}\right) .
$$

This can be written as

$$
\dot{R}^{2} r^{2}=\left(1-k r^{2}\right)(1-\theta) .
$$

Thus the condition that

$$
0<\theta \leq 1
$$

is equivalent to (4.84), in view of our assumptions (4.63) and (4.64). Moreover, since we are assuming (4.62)-(4.65) hold throughout, it is clear that (4.88) is equivalent to (4.84) when $k \leq 0$ as well. When making general statements about FRW-TOV shock-waves, we always assume (4.88) holds.

Proof of Lemma 10: Differentiating (4.34) with respect to $t$ and applying (4.12) yields

$$
\dot{\rho}=\frac{3}{\bar{r}}(\bar{\rho}-\rho) \dot{\bar{r}} .
$$

Solving for $\dot{\rho}$ in (4.7) yields

$$
\dot{\rho}=-\frac{3 \dot{R}}{R}(\rho+p) .
$$

Combining (4.89) and (4.90) thus gives

$$
\dot{\bar{r}}=\dot{R} r \frac{(\rho+p)}{(\rho-\bar{\rho})} .
$$

Differentiating $\bar{r}=R r$ with respect to $t$, using (4.91), and solving for $\dot{r}$ we get

$$
\dot{r}=\frac{\dot{R} r}{R} \frac{(\bar{\rho}+p)}{(\rho-\bar{\rho})} .
$$

Substituting (4.91) and (4.92) into (4.81), we obtain the following equation, which is equivalent to the conservation condition $\left[T^{i j}\right] n_{i} n_{j}=0$ :

$$
0=\left(\frac{1}{1-k r^{2}}\right)(\rho+\bar{p})(p+\bar{\rho})^{2}-\frac{1}{A}(\bar{\rho}+\bar{p})(\rho+p)^{2}+\frac{1}{r^{2} \dot{R}^{2}}(p-\bar{p})(\rho-\bar{\rho})^{2} .
$$


Equation (4.93) expresses conservation at the shock surface (4.34). But by (4.91),

$$
\dot{R}^{2} r^{2}=-A+\left(1-k r^{2}\right)
$$

holds on the shock surface, and using this we can transform (4.93) into the final form (4.82).

For convenience, we summarize the results of this section in the following theorem:

Theorem 8 Assume that FRW and TOV metrics are given that match Lipschitz continuously across the shock surface (4.34) such that (4.62)-(4.65) hold. Then (i)-(iv) of Theorem 4 hold on the shock surface if and only if either (4.69) or (4.82) hold on the shock-surface.

We now use the conservation constraint to solve for $p$ as a function of $\rho, \bar{\rho}$ and $\bar{p}$. Solving (4.82) for $p$ we obtain

$$
p_{+}=\frac{\frac{1}{2}\left\{-(\bar{\rho}+\rho)^{2}+2(\theta-1) \bar{\rho} \bar{p}+2\left(\theta+\frac{1}{\theta}\right) \rho \bar{\rho}+2\left(\frac{1}{\theta}-1\right) \rho \bar{p}+S Q\right\}}{(1-\theta) \rho+\left(2-\theta-\frac{1}{\theta}\right) \bar{p}+\left(1-\frac{1}{\theta}\right) \bar{\rho}}
$$

where

$$
S Q=\left(6 \bar{\rho}^{2} \rho^{2}-4 \rho^{3} \bar{\rho}-4 \bar{\rho}^{3} \rho+\rho^{4}+\bar{\rho}^{4}\right)^{1 / 2}=(\rho-\bar{\rho})^{2} .
$$

Thus we conclude that every TOV solution determines two possible FRW pressures at the shock through the conservation constraint. Since the FRW pressure is constant on the $t=$ const surfaces, these implicitly determine the FRW equations of state $p=p(\rho)$ from the TOV density and pressure. Now the terms in the numerator of (4.95) combine as follows:

$$
-(\bar{\rho}+\rho)^{2}+2\left(\theta+\frac{1}{\theta}\right) \rho \bar{\rho}_{-}^{+}(\rho-\bar{\rho})^{2}=-2\left(2-\theta-\frac{1}{\theta}\right) \rho \bar{\rho}-\left\{2(\rho-\bar{\rho})^{2}\right\}_{-},
$$

where we use the notation that the bracket \{\}$_{-}$is taken to be zero unless we take the minus sign in (4.95), (and corresponding minus sign in (4.97)). Using (4.97) in (4.95) gives

$$
p_{+}=\frac{(\theta-1) \bar{\rho} \bar{p}+\left(\frac{1}{\theta}-1\right) \rho \bar{p}-\left(2-\theta-\frac{1}{\theta}\right) \rho \bar{\rho}-\left\{(\rho-\bar{\rho})^{2}\right\}_{-}}{(1-\theta) \rho+\left(2-\theta-\frac{1}{\theta}\right) \bar{p}+\left(1-\frac{1}{\theta}\right) \bar{\rho}}
$$




$$
\begin{aligned}
& =\frac{-(1-\theta)\{\bar{\rho} \bar{p}-\rho \bar{\rho}\}+\left(\frac{1}{\theta}-1\right)\{\rho \bar{p}+\rho \bar{\rho}\}-\left\{(\rho-\bar{\rho})^{2}\right\}_{-}}{(1-\theta)\{\rho+\bar{p}\}-\left(\frac{1}{\theta}-1\right)\{\bar{p}+\bar{\rho}\}} \\
& =\frac{-(1-\theta) \bar{\rho}(\rho+\bar{p})+\left(\frac{1}{\theta}-1\right) \rho(\bar{p}+\bar{\rho})-\left\{(\rho-\bar{\rho})^{2}\right\}_{-}}{(1-\theta)\{\rho+\bar{p}\}-\left(\frac{1}{\theta}-1\right)\{\bar{p}+\bar{\rho}\}}
\end{aligned}
$$

which upon multiplying the numerator and denominator by $\theta /(1-\theta)$ yields

$$
\begin{gathered}
p_{+}=\frac{-\theta \bar{\rho}(\rho+\bar{p})+\rho(\bar{\rho}+\bar{p})}{\theta(\rho+\bar{p})-(\bar{\rho}+\bar{p})}, \\
p_{-}=\frac{-\theta \bar{\rho}(\rho+\bar{p})+\rho(\bar{\rho}+\bar{p})-\left\{\frac{\theta}{1-\theta}(\rho-\bar{\rho})^{2}\right\}}{\theta(\rho+\bar{p})-(\bar{\rho}+\bar{p})} .
\end{gathered}
$$

We can further simplify $p_{-}$as follows. First, one can verify the identity

$-\theta \bar{\rho}(\rho+\bar{p})+\rho(\bar{\rho}+\bar{p})-\frac{\theta}{1-\theta}(\rho-\bar{\rho})^{2}=\frac{1}{1-\theta}\left(\theta-\frac{\bar{\rho}+\bar{p}}{\rho+\bar{p}}\right)\left(\theta-\frac{\rho}{\bar{\rho}}\right)(\rho+\bar{p}) \bar{\rho}$.

Substituting this into the numerator of (4.100) yields

$$
\begin{aligned}
p_{-} & =\frac{\left(\frac{1}{1-\theta}\right)\left(\theta-\frac{\bar{\rho}+\bar{p}}{\rho+\bar{p}}\right)\left(\theta-\frac{\rho}{\overline{\bar{p}}}\right)(\rho+\bar{p}) \bar{\rho}}{(\rho+\bar{p})\left(\theta-\frac{\bar{\rho}+\bar{p}}{\rho+\bar{p}}\right)} \\
& =\frac{\theta \bar{\rho}-\rho}{1-\theta} .
\end{aligned}
$$

Thus, if we define the variable

$$
\Theta \equiv \gamma \theta
$$

where

$$
\gamma \equiv \frac{\rho+\bar{p}}{\bar{\rho}+\bar{p}}
$$

then the pressures $p_{+}$and $p_{-}$take the similar forms

$$
\begin{aligned}
& p_{+}=\frac{\Theta \bar{\rho}-\rho}{1-\Theta}, \\
& p_{-}=\frac{\theta \bar{\rho}-\rho}{1-\theta} .
\end{aligned}
$$


In Section 5 we will prove that $p=p_{-}$or $\bar{\rho}>\rho$ and leads to $\frac{d p}{d \rho}<0$, and so can be ruled out as physically unlikely possibilities. An easy calculation gives the equivalent formulation of (4.103) in terms of the TOV pressure $\bar{p}$,

$$
\bar{p}=\frac{\theta \bar{\gamma} \rho-\bar{\rho}}{1-\theta \bar{\gamma}}
$$

where

$$
\bar{\gamma}=\frac{\bar{\rho}+p}{\rho+p} .
$$

The following two theorems follow directly from (4.103).

Let us now interpret an FRW-TOV shock-wave as the leading edge of an explosion in which the FRW solution is on the inside, expanding outward into the static TOV solution. In this case, we can take $\bar{\rho} / \rho<1$ as an entropy condition for such a shock-wave; that is, the density should be greater behind the shock. The following theorem states that $\bar{\rho} / \rho<1$ implies that $p>\bar{p}$ as well, when we take the pressure to be $p=p_{+}$in (4.103), and there is a constraint on the allowable values of $\theta$.

Theorem 9 Assume (4.62)-(4.65), assume that

$$
z \equiv \bar{\rho} / \rho<1
$$

and assume

$$
\bar{\mu} \equiv \frac{\bar{p}}{\bar{\rho}} .
$$

Then $p_{+}>0$ if and only if $p-\bar{p}>0$, and this holds if and only if $\theta_{1} \leq \theta<1$ at the shock, where

$$
\theta_{1} \equiv \theta_{1}(z, \bar{\mu}) \equiv \frac{1}{\gamma}=\frac{\bar{\rho}+\bar{p}}{\rho+\bar{p}}=\frac{1+\bar{\mu}}{1+\bar{\mu} z} z
$$

Since the FRW pressure is determined by the TOV solutions according to (4.103), we now ask what possible pressure jumps can be assigned at an FRW-TOV shock-wave at a given position. The final theorem of this section shows that all possible pressure jumps can be assigned as we vary the value of $\theta$. The pressure jumps that can be assinged at a point can be viewed as possible initial conditions for the subsequent dymanics of an FRW-TOV shock-wave solution. 
Theorem 10 Assume (4.62)-(4.65) and that $z<1$. Then for every choice of positive values for $\bar{\rho}, \bar{p}$ and $\rho$, the pressure $p_{+}$monotonically takes on every value from $[\bar{p},+\infty)$, and the pressure difference $\left(p_{+}-\bar{p}\right)$ monotonically takes on every value from $[0,+\infty)$, as $\theta$ ranges monotonically from $\left[1, \theta_{1}\right)$.

Proof: When $\rho>\bar{\rho}$, it follows immediately form (4.102) and (4.103) that $p_{+}>0$ if and only if $\theta>\theta_{1}$. To see this, note that the numerator in (4.103) is always negative because

$$
\gamma \theta z=\frac{1+\bar{\mu} z}{1+\bar{\mu}} \gamma<1
$$

when $z<1$. Thus by (4.103), $p_{+}>0$ if and only if $\gamma \theta>1$. Furthermore, if $\bar{\rho}$, $\bar{p}$ and $\rho$ are fixed, then $p$ varies monotonically from $\bar{p}$ to $\infty$ as $\theta$ varies from +1 to $\theta_{1}$ because $p_{\theta}<0$, and when $\theta=1$,

$$
p_{+}=\frac{\frac{\rho+\bar{p}}{\bar{\rho}+\bar{p}}-\rho}{1-\frac{\rho+\bar{p}}{\bar{\rho}+\bar{p}}}=\bar{p} .
$$

We can perform a similar analysis on the difference $\left(p_{+}-\bar{p}\right)$, because, as is easily shown,

$$
p_{+}-\bar{p}=\left(\frac{1-\theta}{\gamma \theta-1}\right)(\bar{\rho}+\bar{p}) \gamma .
$$

This completes the proofs of the Theorems 9 and 10

Another direct consequence of (4.103), (4.104) is that if $A>0$ and $\theta<1$, then when $\rho>\bar{\rho}$, the only shock-waves with positive pressure must satisfy $p=p_{+}$and

$$
\Theta \equiv \gamma \theta>1
$$

In this case (4.102) implies

$$
\rho>\frac{1}{\theta} \rho+\left(1+\frac{1}{\theta}\right) \bar{p} .
$$




\section{A Class of Solutions Modeling Blast Waves in GR}

\section{$5.1 \quad$ Introduction}

In this section we use the theory developed in Section 4 to construct a class of exact, spherically symmetric, shock-wave solution of the Einstein equations for a perfect fluid. The solutions are obtained by matching a Friedman-Roberson-Walker metric (4.1) to a static Tolman-OppenheimerVolkoff metric (4.10) across a shock-wave interface. This is in the spirit of the Oppenheimer-Snyder solution, except, in contrast to the OppenheimerSnyder model, the pressure $p$ is non-zero. These shock-wave solutions can be interpreted as simple models for the general relativistic version of an explosion into a static, singular, isothermal sphere. It is interesting to keep in mind that shock-waves introduce time-irreversiblity, loss of information, decay, dissipation, and increase of entropy into the dynamics of a perfect fluid in general relativity.

The FRW metric is a uniformly expanding (or contracting) solution of the Einstein gravitational field equations which is generally accepted as a cosmological model for the universe. The TOV solution is a time-independent solution which models the interior of a star. Both metrics are spherically symmetric, and both are determined by a system of ODE's that close when an equation of state $p=p(\rho)$ for the fluid is specified. In the solutions that we construct below, one can imagine the FRW metric as an exploding inner core, (of a star or the universe as a whole), and the boundary of this inner core is a shock surface that is driven by the expansion behind the shock into the outer, static, TOV solution, which we imagine as the outer layers of a star, or the outer regions of the universe. In these solutions, the shock-wave emerges from $\bar{r}=0$ at the initial (Big Bang) singularity in the FRW metric, and so broadly speaking, one can interpret these examples as providing a scenario by which the Big Bang begins with a shock-wave explosion.

The outer static TOV solutions that appear beyond the shock-wave in the examples below, are the general relativistic version of a static isothermal sphere because the metric entries are time-independent, and the constant sound speed can be interpreted as modeling a gas at constant temperature. It is singular because it has an inverse-square density profile, and thus the 
density and pressure tend to $\infty$ at the center of the sphere. The Newtonian version of a static singular isothermal sphere is well known, and is relevant to theories of how stars form from gaseous clouds, [2]. The idea in the Newtonian case goes as follows: a star begins as a diffuse cloud of gas which slowly contracts under its own gravitational force by radiating energy out through the gas cloud as gravitational potential energy is converted into kinetic energy. This contraction continues until the gas cloud reaches the point where the mean free path for transmission of light is small enough that light is scattered, instead of transmitted, through the cloud. The scattering of light within the gas cloud has the effect of equalizing the temperature within the cloud. At this point the gas begins to drift toward the most compact configuration of the density that balances the pressure when the equation of state is isothermal; namely, it drifts toward the configuration of a static, singular, isothermal sphere. Since this solution in the Newtonian case is also inverse square in the density and pressure, the density tends to infinity at the center of the sphere, and this ingnites thermonuclear reactions. The result is a shock-wave explosion emanating from the center of the sphere, and this signifies the birth of the star. One can interpret the exact solutions constructed below as general relativistic versions of such shock-wave explosions.

In the construction we assume that the FRW and TOV solutions both have isothermal equations of state, but at different temperatures. That is, we assume $p=\sigma \rho$ in the FRW solution, and $\bar{p}=\bar{\sigma} \bar{\rho}$ in the TOV solution, where both the inner FRW sound speed $\sqrt{\sigma}$ and the outer TOV sound speed $\sqrt{\bar{\sigma}}$ are assumed to be constant. Here $p$ denotes the fluid pressure and $\rho$ the mass-energy density, and again we let the unbarred and barred variables refer to the standard coordinate systems for the the FRW and TOV metrics (4.1), (4.10), respectively. We assume throughout that the speed of light $c=1$. The construction is based on exact solutions of FRW and TOV type that exist for these special equations of state. In Section 4, (4.34), we showed that in general the shock position $\bar{r}=\bar{r}(t)$ is given implicitly by the equation $M(\bar{r})=\frac{4 \pi}{3} \rho(t) \bar{r}^{3}$, where $M(\bar{r})$ denotes the total TOV mass inside radius $\bar{r}$, and $\rho(t)$ is the FRW density at the shock. For the exact solutions with constant sound speed constructed here, the shock surface condition implies that $\rho=3 \bar{\rho}$ across the shock. Moreover, in order that conservation of energy and momentum hold across the shock, we show that the sound speeds must be related by an algebraic equation of the form $\bar{\sigma}=H(\sigma)$, where $H^{\prime}(\sigma)>0$, $H(0)=0$, and $H(\sigma)<\sigma$, c.f. Figure 1. Since, at the shock, the inner FRW 
sound speed and density exceed the outer TOV sound speed and density, respectively, we conclude that the out-going shock-wave is the stable one, and the larger sound speed in the FRW metric is interpreted as modeling an isothermal equation of state at a higher temperature (consistent with the heating of the fluid by the shock-wave). In the limit $\sigma \rightarrow 0$, the model recovers the Newtonian limit of low velocities and weak gravitational fields.

We verify that there exist two distinguished values of $\sigma, \sigma_{1} \approx .458<$ $\sigma_{2}=\sqrt{5} / 3 \approx .745$, such that, if $0<\sigma<1$, then the Lax characteristic condition (that characteristics impinge on the shock, [14]), is satisfied if and only if $0<\sigma<\sigma_{1}$; and the shock speed is less than the speed of light if and only if $0<\sigma<\sigma_{2}$. A calculation gives $\bar{\sigma}_{1} \equiv H\left(\sigma_{1}\right) \approx .161$, and $\bar{\sigma}_{2} \equiv H\left(\sigma_{2}\right) \approx .236$. We conclude that for $\sigma$ between $\sigma_{1}$ and $\sigma_{2}$, a new type of shock-wave appears in which the shock is supersonic relative to the fluid on both sides of the shock. Thus, in this theory, a fluid with a sound speed no larger than $\sqrt{\sigma_{2}} \approx \sqrt{.745}$ can drive shock-waves with speeds all the way up to the speed of light. The time-reversal and stability properties of these shocks when $\sigma_{1}<\sigma<\sigma_{2}$ remains to be investigated.

Since Lax type shock-waves are time-irreversible solutions of the equations due to the increase of entropy (in a generalized sense, c.f. [27]), and consequent loss of information (effected by the impinging of characteristics on the shock), we infer from the mathematical theory of shock-waves that when $0<\sigma<\sigma_{1}$, many solutions must decay time asymptotically to the same shock-wave. Thus, in contrast to the pure FRW solution, in these models one should not expect a unique time-reversal of the solution all the way back to the initial Big Bang singularity when the sound speed lies in the range $0<\sigma<\sigma_{1}$.

Note that the TOV solution when $\bar{p}=\bar{\sigma} \bar{\rho}$ is, by itself, of limited physical value because $\bar{p}=\infty$ at $\bar{r}=0$. One can interpret this as saying that this exact solution is unstable because it requires an infinite pressure at $\bar{r}=0$ to "hold it up". In contrast, the shock-wave solution here remove the singularity at $\bar{r}=$ 0 , (for times after some initial time), and so the construction demonstrates that a shock-wave in the core can supply the pressure required to stabalize a TOV solution by holding it up. 


\subsection{An Exact Solution of TOV Type}

We now construct exact solutions of TOV type which represent the general relativistic version of static, singular isothermal spheres. First assume the equation of state

$$
\bar{p}=\bar{\sigma} \bar{\rho}
$$

for the TOV metric, and assume that the density is of form

$$
\bar{\rho}(\bar{r})=\frac{\gamma}{\bar{r}^{2}},
$$

for some constant $\gamma$. In this case, an exact solution of TOV type was first found by Tolman ${ }^{9}$, [40]; namely, by (4.14),

$$
M(\bar{r})=4 \pi \gamma \bar{r} .
$$

Putting (5.1)-(5.3) into (4.13) and simplifying, yields the identity

$$
\gamma=\frac{1}{2 \pi \mathcal{G}}\left(\frac{\bar{\sigma}}{1+6 \bar{\sigma}+\bar{\sigma}^{2}}\right) .
$$

From (4.11), we obtain

$$
A=1-8 \pi \mathcal{G} \gamma .
$$

To solve for $B$, start with (4.15) and write

$$
\frac{1}{B} \frac{d B}{d \rho} \frac{d \rho}{d \bar{r}}=-\frac{2 \bar{\sigma}}{(1+\bar{\sigma}) \bar{\rho}} \frac{d \bar{\rho}}{d \bar{r}}
$$

which simplifies to

$$
\frac{d B}{B}=-\frac{2 \bar{\sigma}}{(1+\bar{\sigma})} \frac{d \bar{\rho}}{\bar{\rho}} .
$$

This equation has the explicit solution

$$
B=B_{0}\left(\frac{\bar{\rho}}{\bar{\rho}_{0}}\right)^{-\frac{2 \bar{\sigma}}{1+\bar{\sigma}}}=B_{0}\left(\frac{\bar{r}}{\bar{r}_{0}}\right)^{\frac{4 \bar{\sigma}}{1+\bar{\sigma}}} .
$$

\footnotetext{
${ }^{9}$ In the case $\bar{\sigma}=1 / 3$, this solution was re-discovered by Misner and Zapolsky, c.f. [42], page 320 .
} 
By rescaling the time coordinate, we can take $B_{0}=1$ at $\bar{r}_{0}=1$, in which case (5.8) reduces to

$$
B=\bar{r}^{\frac{4 \bar{\sigma}}{1+\bar{\sigma}}} .
$$

We conclude that when (5.4) holds, (5.1)-(5.5) and (5.8) provide an exact solution of the Einstein field equations (2.16) of TOV type. Note that since $\sqrt{\bar{\sigma}}$ is the sound speed of the fluid, (5.1)-(5.3) provide exact solutions for any sound speed $0 \leq \bar{\sigma} \leq 1$. Note also that when $\bar{\sigma}=1 / 3$, the extreme relativistic limit for free particles, [42], (5.4) yields $\gamma=\frac{3}{56 \pi \mathcal{G}}$, (c.f., [42], equation (11.4.13)). These exact solutions by themselves are not so interesting physically because the density and pressure are infinite at $\bar{r}=0$ at every value of time. Our shock-wave construction, given below, removes the singularity at $\bar{r}=0$ in these solutions, after some initial time.

\subsection{An Exact Solution of FRW Type}

We now construct exact solutions of FRW type. We restrict to the case $k=0$ in (4.1), so that the metric takes the simple (conformally flat) form

$$
d s^{2}=-d t^{2}+R^{2}(t)\left\{d r^{2}+r^{2} d \Omega^{2}\right\}
$$

Now assume an arbitrary equation of state of the form $p=p(\rho)$. We will obtain a closed form solution of the Einstein equations (2.16) in this case. By (4.7)-(4.8), it suffices to solve the system of two ODE's

$$
\dot{R}^{2}=\frac{8 \pi \mathcal{G}}{3} \rho R^{2},
$$

and

$$
p(\rho)=-\rho-\frac{R \dot{\rho}}{3 \dot{R}} .
$$

Rewrite (5.11) as

$$
\dot{R}={ }_{-}^{+} \sqrt{\frac{8 \pi \mathcal{G} \rho}{3}} R,
$$

and substitute into (5.12) to obtain

$$
p=-\rho_{+}^{-} \frac{\dot{\rho}}{\sqrt{24 \pi \mathcal{G} \rho}} .
$$


(The upper/lower plus-minus signs will always correspond to the two cases represented by the upper/lower plus-minus sign in (5.13), respectively.) The point to be noted here is that when $p=p(\rho)$ is assigned, (5.14) is independent of $R$, and thus we can integrate it explicitly; namely, since

$$
d t=_{+}^{-} \frac{d \rho}{(\rho+p) \sqrt{24 \pi \mathcal{G} \rho}},
$$

we obtain

$$
t-t_{0}=-\int_{\rho_{0}}^{\rho} \frac{d \xi}{(\xi+p(\xi)) \sqrt{24 \pi \mathcal{G} \xi}} .
$$

Formula (5.16) gives $t$ as a function of $\rho$, and we can use this, together with (5.11), to obtain a closed form expression for $R$ as a function of $\rho$. Thus since

$$
\dot{R}=\frac{d \rho}{d t} \frac{d R}{d \rho}=-(\rho+p) \sqrt{24 \pi \mathcal{G} \rho} \frac{d R}{d \rho},
$$

if we combine this with (5.11), we get

$$
\frac{d R}{R}=\frac{-d \rho}{3(\rho+p)},
$$

which has the explicit solution

$$
R=R_{0} \exp \int_{\rho_{0}}^{\rho} \frac{-1}{3(\xi+p(\xi))} d \xi
$$

\subsection{A Class of Exact Shock-Wave Solutions of the Ein- stein Equations}

We now use the theory developed in [30] to match the above TOV and FRW type metrics at a spherical interface across which the metrics join Lipschitz continuously, and such that the conservation constraint (4.34) holds at the interface. The resulting solution is interpreted as a fluid dynamical shockwave in which the increase of entropy in the fluids drives a time-irreversible gravitational wave.

Assume now that the equation of state for the TOV metric is taken to be

$$
\bar{p}=\bar{\sigma} \bar{\rho}
$$


for some constant $\bar{\sigma}$, and that the fixed TOV solution is given by (5.2)(5.5) and (5.8). Then, given an arbitrary FRW metric, our results in [30] imply that we can construct a coordinate mapping $(\bar{t}, \bar{r}) \rightarrow(t, r)$ such that the FRW metric matches the TOV metric Lipschitz continuously across the shock surface (4.34). This applies, in principle, to any equation of state $p=p(\rho)$ chosen for the FRW metric. Using (5.3) and solving for $\rho$ gives $\rho$ on the shock surface $\bar{r}(t)=r(t) R(t)$ :

$$
\rho=\frac{3}{4 \pi} \frac{M}{\bar{r}(t)^{3}}=\frac{3 \gamma}{\bar{r}(t)^{2}}=3 \bar{\rho} .
$$

To meet the additional conservation condition, we restrict to FRW metrics with $k=0$, and we use (4.82) to determine the pressure. Substituting $\Theta=A=1-8 \pi \mathcal{G} \gamma \equiv$ const. into (4.82), we see that the resulting equation is homogeneous of degree three in the $\rho, \bar{\rho}$ and $p, \bar{p}$ variables. Since $\bar{p}=\bar{\sigma} \bar{\rho}$, and

$$
\rho=3 \bar{\rho}
$$

on the shock surface, it is clear from homogeneity that (4.82) can be met if and only if $p=\sigma \rho$ for some constant $\sigma$. Substituting this into (4.82) gives the following relation between $\sigma$ and $\bar{\sigma}$; (c.f. Figure 1)

$$
\bar{\sigma}=\frac{1}{2} \sqrt{9 \sigma^{2}+54 \sigma+49}-\frac{3}{2} \sigma-\frac{7}{2} \equiv H(\sigma) .
$$

Alternatively, we can solve for $\sigma$ in (5.21) and write this relation as

$$
\sigma=\frac{\bar{\sigma}(\bar{\sigma}+7)}{3(1-\bar{\sigma})} .
$$

This guarantees that conservation holds across the shock surface, and thus Theorem 5 holds, and the results of Theorem 4 apply. Note that $H(0)=0$, and to leading order,

$$
\bar{\sigma}=H(\sigma)=\frac{3}{7} \sigma+O\left(\sigma^{2}\right)
$$

as $\sigma \rightarrow 0$. It is easy to verify that within the physical region $0 \leq \sigma, \bar{\sigma} \leq 1$, $H^{\prime}(\sigma)>0$, and $\bar{\sigma}<\sigma$, as would be expected physically because $\rho=3 \bar{\rho}>\bar{\rho}$ at the shock surface. One can verify that when $\sigma=1 / 3$, we have

$$
\bar{\sigma}=\sqrt{17}-4=.1231 \ldots
$$


and when $\sigma=1$, we have

$$
\bar{\sigma}=\frac{\sqrt{112}}{2}-5=.2915 \ldots
$$

We now obtain formulas for $\rho(t), R(t)$ and the shock positions $r(t)$ and $\bar{r}(t)=r(t) R(t)$. Substituting $p=\sigma \rho$ into (5.15) and (5.18) yields

$$
d t=_{+}^{-} \frac{1}{\sqrt{24 \pi \mathcal{G}}(1+\sigma)} \rho^{-\frac{3}{2}} d \rho,
$$

and

$$
\frac{d R}{R}=-\frac{1}{3(1+\sigma)} \frac{d \rho}{\rho} .
$$

Using (5.20) we obtain

$$
\rho^{-\frac{3}{2}} d \rho=-\frac{2}{\sqrt{3 \gamma}} d \bar{r}
$$

Putting this into (5.24) gives

$$
d t={ }_{+}^{-} \frac{1}{(1+\sigma)} \frac{1}{\sqrt{18 \pi \mathcal{G} \gamma}} d \bar{r} .
$$

Integrating equation (5.27) gives the formula for the shock position:

$$
\bar{r}(t)=_{-}^{+} \sqrt{18 \pi \mathcal{G} \gamma}(1+\sigma)\left(t-t_{0}\right)+\bar{r}_{0}
$$

Thus (5.20) gives $\rho$ in terms of $t$ :

$$
\rho(t)=\frac{3 \gamma}{\bar{r}(t)^{2}}=\frac{3 \gamma}{\left({ }_{-}^{+} \sqrt{18 \pi \mathcal{G} \gamma}(1+\sigma)\left(t-t_{0}\right)+\bar{r}_{0}\right)^{2}} .
$$

Finally, we can use (5.25) to obtain $R(t)$, and the shock position $r(t)=$ $\bar{r}(t) R(t)^{-1}$ :

$$
\begin{gathered}
R(t)=R_{0}\left(\frac{\rho}{\rho_{0}}\right)^{-\frac{1}{3(1+\sigma)}}=R_{0}\left(\frac{\bar{r}(t)}{\bar{r}_{0}}\right)^{\frac{2}{3(1+\sigma)}} \\
r(t)=\bar{r}(t) R(t)^{-1}=\bar{r}(t) R_{0}^{-1}\left(\frac{\bar{r}(t)}{\bar{r}_{0}}\right)^{-\frac{2}{3(1+\sigma)}}=\bar{r}_{0} R_{0}^{-1}\left(\frac{\bar{r}(t)}{\bar{r}_{0}}\right)^{\frac{1+3 \sigma}{3+3 \sigma}} .
\end{gathered}
$$


Differentiating (5.28) and (5.31) gives the speeds of the shock $\dot{\bar{r}}$ and $\dot{r}$ in the $(t, \bar{r})$ - and $(t, r)$-coordinate systems, respectively:

$$
\begin{gathered}
\dot{\bar{r}}=3(1+\sigma) \sqrt{\frac{\bar{\sigma}}{1+6 \bar{\sigma}+\bar{\sigma}^{2}}}, \\
\dot{r}=\frac{1+3 \sigma}{R(t)} \sqrt{\frac{\bar{\sigma}}{1+6 \bar{\sigma}+\bar{\sigma}^{2}}},
\end{gathered}
$$

where again, $\bar{\sigma}=H(\sigma)$ is given in (5.21).

Note that the solution (5.28)-(5.31) contains two arbitrary constants $\bar{r}_{0}, R_{0}$ or $r_{0}, R_{0}$, as it should from the initial value problem (5.13), (5.14). Note also that for an outgoing shock-wave, we choose the plus sign in (5.13) and (5.28), and in this case there is a singularity in backward time

$$
t_{*}=t_{0}-\frac{\bar{r}_{0}}{\sqrt{18 \pi \mathcal{G} \gamma}(1+\sigma)} .
$$

As $t \rightarrow t_{*}$, it is clear that $\bar{r} \rightarrow 0, \rho, \bar{\rho}, p, \bar{p}$ all tend to infinity, and $R, r$ tend to zero. If we take this as a cosmological model, then $t=t_{*}$ represents the initial Big Bang singularity in which a shock-wave emerges from $\bar{r}=0$.

We summarize these results in the following theorem:

Theorem 11 Assume an equation of state of the form $\bar{p}=\bar{\sigma} \bar{\rho}$ for the TOV metric, and $p=\sigma \rho$ for the FRW metric, assume (5.21) holds, and take $k=0$. Then the TOV solution given by (5.2), (5.3), (5.5), (5.8), will match the FRW solution given by (5.29), (5.30), across the shock surface (5.28), such that conservation of energy and momentum hold across the surface. The coordinate identification $(t, r) \rightarrow(\bar{t}, \bar{r})$ is given by $\bar{r}=R r$, together with a smooth function $\bar{t}=\bar{t}(t, r)$ whose existence (in a neighborhood of the shock surface) is demonstrated in [30].)

By Theorem 5, all of the equivalencies in Theorem 4 hold across the shock surface. In the next section we show that the shock speeds are less than the speed of light, and we determine when the Lax characteristic conditions hold.

\subsection{The Lax Shock Conditions}

To complete the analysis of our shock-wave solution discussed in the last section, it remains to analyze the shock speed and characteristic speeds on 
both sides of the shock. In classical gas dynamics, characteristics (in the same family of a shock) impinge on the shock from both sides, leading to an increase of entropy and consequent loss of information. This is also the source of the well known time-irreversibility, as well as the stability, of gas dynamical shock-waves. This interpretation carries over to a general system of hyperbolic conservation laws. Indeed, this characteristic condition has been proposed by Lax, $[14,27]$, as a stability criterion for shock- waves in settings other than gas dynamics. This "Lax characteristic condition" can be easily applied in general systems where, either a physical entropy is difficult to work with, or has not been identified, [27]. Since in gas dynamics the density and pressure are always larger behind (stable) shock-waves, and in our example $\rho=3 \bar{\rho}$, (c.f., (5.20)), we restrict our attention to the case of an outgoing shock-wave in which the FRW metric is on the inside and the TOV metric is on the outside of the shock. This is equivalent to taking the plus sign in (5.13), (and the corresponding upper sign in the equations (5.14)-(5.16)), and we therefore restrict our attention to this case.

The goal of this section is to show that, in this case, there exist values $0<\sigma_{1}<\sigma_{2}<1,\left(\sigma_{1} \approx .458, \sigma_{2}=\sqrt{5} / 3 \approx .745\right)$, such that, for $0<\sigma<1$, the Lax characteristic condition holds at the shock if and only if $0<\sigma<\sigma_{1}$; and the shock speed is less than the speed of light if and only if $0<\sigma<\sigma_{2}$. We conclude that our gravitational shock-wave represents a new type of fluid dynamical shock-wave when $\sigma_{2}<\sigma<1$. For the outgoing shock-waves with $\sigma$ in this interval, the shock speed exceeds all of the characteristic speeds on either side of the shock, because both the fast and slow characteristics cross the shock-wave from the TOV side to the FRW side of the shock. Our first result is the following lemma:

Lemma 11 For $0<\sigma<1$, the shock speed, relative to the FRW fluid particles, is given by

$$
s=(1+3 \sigma) \sqrt{\frac{\bar{\sigma}}{1+6 \bar{\sigma}+\bar{\sigma}^{2}}} \equiv s(\sigma),
$$

where $s(\sigma)$ is the function of $\sigma$ obtained by substituting (5.21) for $\bar{\sigma}$ in (5.35).

The function $s(\sigma)$ is plotted in Figure 2. By numerical calculation we obtain that $1-s(\sigma)$ is monotone for $0<\sigma<1$, and becomes negative above $\sigma=\sigma_{2}$, where, using computer algebra, we find

$$
\sigma_{2}=\sqrt{5} / 3 \approx .745
$$


Therefore, by general covariance, the following theorem is a consequence of Lemma 11:

Theorem 12 For $0<\sigma<1$, the shock speed is less than the speed of light if and only if $\sigma<\sigma_{2}$.

To prove Lemma 11, we recall that the "speed" of a shock is a coordinate dependant quantity that can be interpreted in a special relativistic sense at a point $P$ in coordinate systems for which $g_{i j}(P)=\operatorname{diag}(-1,1,1,1)$. (We call such coordinate frames "locally Minkowskian" to distinguish these from "locally Lorentzian" frames in which $g_{i j, k}(P)=0$ as well. Since we are dealing only with velocities and not accelerations, we do not need to invoke the additional condition $g_{i j, k}(P)=0$ for a local Lorentzian coordinate frame in order to recover a special relativistic interpretation for velocities.) In such coordinate frames, a "speed" at $P$ transforms according to the special relativistic velocity transformation law when a Lorentz transformation is performed. We now determine the shock speed at a point $P$ on the shock in a locally Minkowskian frame that is co-moving with the FRW metric. To this end, let $(t, r)$-coordinates correspond to the FRW metric with $k=0$ in (5.10). Let $(t, \tilde{r})$-coordinates correspond to a locally Minkowskian system obtained from $(t, r)$ by a transformation of the form $r=\varphi(\tilde{r})$, so that

$$
\begin{gathered}
d s^{2}=-d t^{2}+R(t)^{2}\left(\left(\varphi^{\prime}\right)^{2} d \tilde{r}^{2}+\varphi^{2} d \Omega^{2}\right) \\
d s^{2}=\frac{\varphi^{2}}{\tilde{r}^{2}}\left(-\frac{\tilde{r}^{2}}{\varphi^{2}} d t^{2}+\frac{R^{2}\left(\varphi^{\prime}\right)^{2} \tilde{r}^{2}}{\varphi^{2}} d \tilde{r}^{2}+\tilde{r}^{2} d \Omega^{2}\right) .
\end{gathered}
$$

Choose $\varphi$ so that $\frac{\varphi^{2}}{\tilde{r}^{2}}=1$ and $\frac{R^{2}\left(\varphi^{\prime}\right)^{2} \tilde{r}^{2}}{\varphi^{2}}=1$ at the point $P$; i.e., at $P=P(t, r)$, set $\varphi(r)=\tilde{r}$, and $\varphi^{\prime}(r)=\frac{1}{R(t)}$. Thus, in the $(t, \tilde{r})$-coordinates,

$$
d s^{2}=-d t^{2}+d \tilde{r}^{2}+\tilde{r}^{2} d \Omega^{2}
$$

at the point $P$, and so the $(t, \tilde{r})$-coordinates represent the class of locally Minkowskian coordinate frames that are fixed relative to the fluid particles of the FRW metric at the point $P$. (That is, any two members of this class of coordinate frames will differ by higher order terms that do not affect the calculation of velocities at $P$.) Therefore, the speed $\frac{d \tilde{r}}{d t}$ of a particle in $(t, \tilde{r})-$ coordinates gives the value of the speed of the particle relative to the FRW 
fluid in the special relativistic sense. Since

$$
\frac{d r}{d t}=\frac{d r}{d \tilde{r}} \frac{d \tilde{r}}{d t}=\varphi^{\prime} \frac{d \tilde{r}}{d t}=\frac{1}{R} \frac{d \tilde{r}}{d t},
$$

we conclude that if the speed of a particle in $(t, r)$-coordinates is $\frac{d r}{d t}$, then its geometric speed relative to observers fixed with the FRW fluid, (and hence also fixed relative to the radial coordinate $r$ of the FRW metric because the fluid is co-moving), is equal to $R \frac{d r}{d t}$.

Now consider the shock-wave (5.31), which moves with speed, (c.f. (5.33)),

$$
\frac{d r}{d t} \equiv \dot{r}=\frac{1+3 \sigma}{R(t)} \sqrt{\frac{\bar{\sigma}}{1+6 \bar{\sigma}+\bar{\sigma}^{2}}} .
$$

Then by (5.37), the speed of the shock $s$ relative to the FRW fluid particles must be given by (5.35). A graph of $s(\sigma)$ is given in Figure 2, from which we conclude that the shock speed moves with a speed less that one relative to the FRW fluid if and only if $\sigma<\sigma_{2}$ holds; and for $0<\sigma<1, s(\sigma)=1$ if and only if $\sigma=\sigma_{2}$, where numerical symbolic algebra gives $\sigma_{2}=\sqrt{5} / 3 \approx .745$. This completes the proof of Lemma 11.

We next determine when the Lax characteristic condition holds at the shock. To this end, we first determine the speed of the characteristics relative to the fixed FRW fluid particles. By (5.37), the characteristic speeds on the FRW side of the shock must equal the sound speeds ${ }_{-}^{+} \sqrt{\sigma}$ in the $(t, \tilde{r})-$ coordinate frame, because the FRW fluid is co-moving with respect to the $(t, \tilde{r})$-coordinates. (The characteristic speed is obtained from the fluid speed and sound speed by the special relativistic summation formula for velocities, [29].) We conclude that the FRW characteristic speeds, $\tilde{\lambda}_{F R W}^{-}, \tilde{\lambda}_{F R W}^{+}$(the speeds of the characteristics relative to the FRW fluid), are given, respectively, by the formula

$$
\tilde{\lambda}_{F R W}^{+} \equiv_{-}^{+} \frac{d \tilde{r}}{d t}={ }_{-}^{+} \sqrt{\sigma}
$$

By (5.37),

$$
\lambda_{F R W}^{+}=\tilde{\lambda}_{F R W}^{+} \frac{1}{R}={ }_{-}^{+} \frac{\sqrt{\sigma}}{R} .
$$

Thus, since the $(t, r)$-coordinates are also co-moving with the fluid, the sound waves in the $(t, r)$-coordinates of the FRW metric must move at coordinate 
speed

$$
\frac{d r}{d t}={ }_{-}^{+} \frac{\sqrt{\sigma}}{R}
$$

We refer to the,-+ characteristics as being in the 1,2-characteristic families, respectively. Now in the one space-one time-dimensional theory of conservation laws, the Lax characteristic condition states that the characteristic curves in the family of the shock, impinge upon the shock from both sides, while all other characteristic curves cross the shock, c.f. [27]. Since in our example, the shock is outward moving with respect to $r$ and $\bar{r}$, it follows that on the FRW side, only the 2-characteristic can impinge on the shock, and thus we must identify the shock-wave as a 2-shock. Thus the Lax characteristic condition must be interpreted as meaning that the following inequalities hold:

$$
s<\tilde{\lambda}_{F R W}^{+}
$$

and

$$
\tilde{\lambda}_{T O V}^{+}<s .
$$

Here $\tilde{\lambda}_{T O V}^{+}$refers to the speed of the faster characteristic on the TOV side of the shock as measured in the $(t, \tilde{r})$-coordinate system, which is related to the $(\bar{t}, \bar{r})$-coordinate system through the $(t, r) \rightarrow(\bar{t}, \bar{r})$ coordinate identification. By (5.35) and (5.39), (5.40) is equivalent to the condition

$$
\tilde{\lambda}_{F R W}^{+}-s(\sigma) \equiv \Delta(\sigma)=\sqrt{\sigma}-(1+3 \sigma) \sqrt{\frac{\bar{\sigma}}{1+6 \bar{\sigma}+\bar{\sigma}^{2}}}>0 .
$$

A numerical plot of the function $\Delta(\sigma)$, given in Figure 3, shows that $\Delta(\sigma)$ changes from positive to negative at the unique point $\sigma=\sigma_{1}$, where

$$
\sigma_{1} \approx .458
$$

We are now ready to prove the following theorem:

Theorem 13 For $0<\sigma<1$, the Lax characteristic conditions (5.40), (5.41) hold across the shock if and only if $0<\sigma<\sigma_{1}$.

Since (5.40) follows from (5.42) and (5.43), the proof of Theorem 13 will be complete once we prove the following lemma which immediately implies (5.41). 
Lemma 12 The inequality

$$
\tilde{\lambda}_{T O V}^{-}<\tilde{\lambda}_{T O V}^{+}<0
$$

holds for all $0<\sigma<1$.

The next theorem is another immediate consequence of Lemma 5.5:

Theorem 14 If $\sigma_{1}<\sigma<\sigma_{2}$, then the following inequalities hold:

$$
\tilde{\lambda}_{F R W}^{-}<\tilde{\lambda}_{F R W}^{+}<s(\sigma)
$$

and

$$
\tilde{\lambda}_{T O V}^{-}<\tilde{\lambda}_{T O V}^{+}<s(\sigma) .
$$

Note that when $\sigma_{1}<\sigma<\sigma_{2}$, (5.45),(5.46) describe a new kind of shock-wave in which the 1- and 2-characteristics both cross the shock because the shock speed exceeds the characteristic speeds on both sides of the shock. This occurs even though the sound speeds and shock speed all remain less than the speed of light. In words, Theorem 14 states that in General Relativity, a sound speed $\sqrt{\sigma} \approx \sqrt{.744}$ can drive the shock speed all the way up to the speed of light.

It remains only to give the proof of Lemma 5.5. Let $\overline{\mathbf{u}}$ denote the velocity vector for the fluid on the TOV side of the shock, and let $\alpha=0,1$ refer to components in the $(\bar{t}, \bar{r})$-coordinate frame and $i=0,1$ to components in the $(t, r)$-coordinate frame. Then a velocity vector tangent to the particle paths of the fluid on the TOV side of the shock is given by $\left(\bar{u}^{0}, \bar{u}^{1}\right)=(1,0)$ in barred coordinates, because the fluid is co-moving relative to the barred coordinate system on the TOV side of the shock; for brevity we write $\bar{u}^{\alpha}=(1,0)^{\alpha}$. (Since our aim is to compute the characteristic speed, which is a ratio of two vector components, a tangent vector of any length will suffice.) Let $x^{i} \equiv(t, r)^{i}$ and $\bar{x}^{\alpha} \equiv(\bar{t}, \bar{r})^{\alpha}$. Then

$$
u^{i}=\frac{\partial x^{i}}{\partial \bar{x}^{\alpha}} \bar{u}^{\alpha}=\frac{\partial x^{i}}{\partial \bar{x}^{0}} \bar{u}^{0}=\frac{\partial x^{i}}{\partial \bar{x}^{0}} .
$$

Thus the speed of the TOV fluid as measured in the FRW coordinates $(t, r)$ is given by

$$
u \equiv \frac{u^{1}}{u^{0}}=\frac{\frac{\partial x^{1}}{\partial \bar{x}^{0}}}{\frac{\partial x^{0}}{\partial \bar{x}^{0}}}=\frac{\frac{\partial r}{\partial t}(\bar{t}, \bar{r})}{\frac{\partial t}{\partial t}(\bar{t}, \bar{r})} .
$$


But,

$$
\frac{\partial t}{\partial \bar{t}}(\bar{t}, \bar{r})=\frac{1}{\frac{\partial \bar{t}}{\partial t}(t, \bar{r})},
$$

so

$$
u \equiv \frac{\partial r}{\partial \bar{t}}(\bar{t}, \bar{r}) \cdot \frac{\partial \bar{t}}{\partial t}(t, \bar{r})=\frac{\partial r}{\partial t}(t, \bar{r}) .
$$

Since

$$
r(t, \bar{r})=\frac{\bar{r}}{R(t)},
$$

and this holds in a neighborhood of the shock surface, we have

$$
u=\frac{\partial r}{\partial t}(t, \bar{r})=\frac{\partial}{\partial t} \frac{\bar{r}}{R(t)}=-\frac{\bar{r} \dot{R}}{R^{2}} .
$$

But by (5.30),

$$
\dot{R}=\frac{2}{r(t)} \sqrt{\frac{\bar{\sigma}}{1+6 \bar{\sigma}+\bar{\sigma}^{2}}},
$$

so

$$
u \equiv-\frac{\bar{r}}{R(t)^{2}} \dot{R}(t)=-\frac{2}{R} \sqrt{\frac{\bar{\sigma}}{1+6 \bar{\sigma}+\bar{\sigma}^{2}}} .
$$

Thus by (5.37),

$$
\tilde{u}=-2 \sqrt{\frac{\bar{\sigma}}{1+6 \bar{\sigma}+\bar{\sigma}^{2}}},
$$

and this gives the TOV fluid speed in the locally Minkowskian frame which is fixed with the FRW fluid particles. But $\sqrt{\bar{\sigma}}$ is the sound speed for the TOV metric; thus $\sqrt{\bar{\sigma}}$ is the sound speed as measured in the frame obtained from the $(t, \tilde{r})$ coordinates by a Lorentz transformation for $\tilde{u}$. Therefore, to obtain the TOV characteristic speed $\tilde{\lambda}_{T O V}^{+}$in the frame $(t, \tilde{r})$, we use the relativistic addition of velocities formula:

$$
\tilde{\lambda}_{T O V}^{+}=\frac{d \tilde{r}}{d t}=\frac{\tilde{u}+\sqrt{\bar{\sigma}}}{1+\tilde{u} \sqrt{\bar{\sigma}}},
$$

and this implies that

$$
\lambda_{T O V}^{+}=\frac{d r}{d t}=\frac{1}{R} \frac{\tilde{u}+\sqrt{\bar{\sigma}}}{1+\tilde{u} \sqrt{\bar{\sigma}}} .
$$


We now calculate $\tilde{\lambda}_{T O V}^{+}$. By $(5.54)$, we have

$$
\tilde{\lambda}_{T O V}^{+}=-\frac{2-\sqrt{1+6 \bar{\sigma}+\bar{\sigma}^{2}}}{\sqrt{1+6 \bar{\sigma}+\bar{\sigma}^{2}}-2 \bar{\sigma}} \sqrt{\bar{\sigma}} \equiv \tilde{\lambda}_{T O V}^{+}(\sigma),
$$

where again we use (5.21) to eliminate $\bar{\sigma}$ in favor of $\sigma$. A numerical plot of $\tilde{\lambda}_{T O V}^{+}(\sigma)$ vs $\sigma$ is given in Figure 4 . This verifies that $\tilde{\lambda}_{T O V}^{+}(\sigma)<0$ for $0<\sigma<1$, and thus completes the proof of Lemma 5.5 in light of the inequality $\tilde{\lambda}_{T O V}^{-}<\tilde{\lambda}_{T O V}^{+}$.

\subsection{Concluding Remarks}

Note that these examples provide a theory of inherently strong shock-waves because the condition $\rho=3 \bar{\rho}$ implies that $[\rho] \rightarrow 0$ iff $\rho \rightarrow 0$, the latter being a singular limit, c.f. [27].

Note also that when $k>0$, the FRW-TOV shocks-wave solutions described in Section 4 reduce to the well known model of Oppenheimer and Snyder $(\mathrm{O}-\mathrm{S})$ when $\bar{p} \equiv 0$. It is interesting to note, however, that the $O$ $S$ model reduces to flat Minkowski space when we take $k \rightarrow 0$ in the $O-S$ solution, (see Weinberg, [42], page 344, equations (11.9.23) and (11.9.21)). Moreover, when we take $\bar{\sigma} \rightarrow 0$ in our solution (5.28)-(5.31), we also get flat Minkowski space. However, the first limit is singular (because $\dot{R}=0$ implies $R \equiv$ const. when $k=0$, c.f. [42], page 344, equation (11.9.22)); the second limit is only one way to impose $\bar{\rho}=0$. Indeed, we can obtain a new, time-reversible O-S type contact discontinuity for the case $k=0$ by noting first from (4.82) that $\bar{\rho} \equiv 0 \equiv \bar{p}$ implies $p=0$, and thus we can integrate (5.16) and (5.19) in the case $p=0$ to obtain the formulas

$$
\begin{gathered}
\rho(t)=\frac{1}{\left({ }_{-}^{+} \sqrt{6 \pi \mathcal{G}}\left(t-t_{0}\right)+\frac{1}{\sqrt{\rho_{0}}}\right)^{2}}, \\
R(t)=R_{0}\left(\frac{\rho(t)}{\rho_{0}}\right)^{-1 / 3} .
\end{gathered}
$$

The shock surface is then given by

$$
\bar{r}(t)=\left(\frac{3}{4 \pi} \frac{M}{\rho(t)}\right)^{1 / 3}
$$


where $M \equiv$ const when we assume empty space $\bar{\rho} \equiv 0 \equiv \bar{p}$. We conclude that (5.58)-(5.60) defines a non-trivial, time-reversible general relativistic model that corresponds to the exact shock-wave solution given in (5.28)-(5.31), and thus defines a new O-S type model of gravitational collapse, c.f., [42], page 345, equation (11.9.25).

We note also that once values for $\sigma$ and $\bar{\sigma}=H(\sigma)$ are specified, the formulas (5.28)-(5.31) determine a unique shock-wave solution despite the appearance of two free parameters, say $R_{0}$ and $\bar{r}_{0}$. To see this, note that after fixing the shock position $\bar{r}_{0}$, the freedom in $R_{0}$ is only a coordinate freedom due to the fact that $R(t) \rightarrow \alpha^{-1} R(t)$ under the coordinate rescaling $r \rightarrow \alpha r$ in the FRW metric (5.10) when $k=0$.

\section{A Shock Wave Formulation of the Einstein Equations}

\subsection{Introduction}

In this section we show that Einstein equations (2.25)-(2.28) are weakly equivalent to the system of conservation laws with time dependent sources (2.48),(2.49), so long as the metric is in the smoothness class $C^{0,1}$, and $T$ is in $L^{\infty}$. Inspection of equations (2.25)-(2.28) shows that it is in general not possible to have metrics smoother than Lipschitz continuous, (that is,

smoother than $C^{0,1}$ at shocks), when the metric is written in the standard gauge. Indeed, at a shock wave where $T$ is discontinuous, $A_{r}, B_{r}$ and $B_{t}$ all have jump discontinuities.

As stated in Section 1, a spacetime metric $g$ is said to be spherically symmetric if it takes the general form, $[42,41,11,22]$,

$$
d s^{2}=g_{i j} d x^{i} d x^{j} \equiv-A(r, t) d t^{2}+B(r, t) d r^{2}+2 D(r, t) d t d r+C(r, t) d \Omega^{2},
$$

where the components $A, B, C$ and $D$ of the metric are assumed to be functions of the radial and time coordinates $r$ and $t$ alone, $d \Omega^{2} \equiv d \theta^{2}+$ $\sin ^{2}(\theta) d \phi^{2}$ denotes the line element on the 2 -sphere, and $\mathbf{x} \equiv\left(x^{0}, \ldots, x^{3}\right) \equiv$ $(t, r, \theta, \phi)$, denotes the underlying coordinate system on spacetime. In this case we assume that the 4 -velocity $\mathbf{w}$ is radial, by which we mean that the $x$-components of $\mathbf{w}$ are given by 


$$
w^{i}=\left(w^{0}(r, t), w^{1}(r, t), 0,0\right), \quad i=0, \ldots, 3, \quad \text { respectively, }
$$

for some functions $w^{0}$ and $w^{1}$.

Now in Section 1 we showed that, in general, there always exists a coordinate transformation $(r, t) \rightarrow(\bar{r}, \bar{t})$ that takes an arbitrary metric of form (6.1) over to one of the form, [42],

$$
d s^{2}=g_{i j} d x^{i} d x^{j} \equiv-A(r, t) d t^{2}+B(r, t) d r^{2}+r^{2} d \Omega^{2} .
$$

A metric of form (6.3) is said to be in the standard Schwarzschild coordinates, or (standard coordinate gauge), and it is our purpose here to establish the weak formulation of the Einstein equations (2.25)-(2.28) for metrics of the form (6.3) in the case when $A$ and $B$ are finite, and satisfy $A B \neq 0$.

In Sections 2 and 3 we introduce and verify the equivalence of several weak formulations of the Einstein equations that allow for shock waves, and that are valid for metrics of form (6.3), in the smoothness class $C^{0,1}$. In Section 4 , we show that these equations are weakly equivalent to the system (2.48)-(2.49) of conservation laws with time dependent sources. This is the starting point for the existence theory set out in [10].

\subsection{The Einstein Equations for a Perfect Fluid with Spherical Symmetry}

In this section we study the system of equations obtained from the Einstein equations under the assumption that the spacetime metric $g$ is spherically symmetric. So assume that the gravitational metric $g$ is of the form (6.3), and to start, assume that $T^{i j}$ is any arbitrary stress tensor. To obtain the equations for the metric components $A$ and $B$ implied by the Einstein equations (2.15), plug the ansatz (6.3) into the Einstein equations (2.15). The resulting system of equations (2.25)-(2.28) is obtained using MAPLE. Equations (2.25)-(2.28) represent the $(0,0),(0,1),(1,1)$ and $(2,2)$ components of $G^{i j}=\kappa T^{i j}$, respectively, (as indexed by $T$ on the RHS of each equation). The $(3,3)$ equation is a multiple of the $(2,2)$ equation, and all remaining components are identically zero. (Note that MAPLE defines the curvature tensor to be minus one times the curvature tensor defined in (2.14).)

We are interested in solutions of (2.25)-(2.28) in the case when shock waves are present. Since $A$ and $B$ have discontinuous derivatives when shock 
waves are present, it follows that (2.28), being second order, cannot hold classically, and thus equation (2.28) must be taken in the weak sense, that is, in the sense of the theory of distributions. To get the weak formulation of (2.28), multiply through by $A B^{2}$ to clear away the coefficient of the highest (second) order derivatives, then multiply through by a test function and integrate the highest order derivatives once by parts. It follows that if the test function is in the class $C_{0}^{1,1}$, (that is, one continuous derivative that is Lipschitz continuous, the subscript zero denoting compact support), and if the metric components $A$ and $B$ are in the class $C^{0,1}$, and $T^{i j}$ is in class $L^{\infty}$, then all terms in the integrand of the resulting integrated expression are at most discontinuous, and so all derivatives make sense in the classical pointwise a.e. sense.

In order to account for initial and boundary conditions in the weak formulation, it is standard to take the test function $\phi$ to be nonzero at $t=0$ or at the specified boundary. In this case, when we integrate by parts to obtain the weak formulation, the boundary integrals are non-vanishing, and their inclusion in the weak formulation represents the condition that the boundary values are taken on in the weak sense. Thus, for example, if the boundary is $r=r_{0} \geq 0$, we say $\phi \in C_{0}^{1,1}\left(r \geq r_{0}, t \geq 0\right)$ to indicate that $\phi$ can be nonzero initially and at the boundary $r=r_{0}$, thereby implicitly indicating that boundary integrals will appear in the weak formulation based on such test functions.

We presently consider various equivalent weak formulations of equations (2.25)-(2.28), and we wish to include the equivalence of the weak formulation of boundary conditions in the discussion. Thus, in order to keep things as simple as possible, we now restrict to the case of weak solutions of (2.25)(2.28) defined on the domain $r \geq r_{0} \geq 0, t \geq 0$, and we always assume that test functions $\phi$ lie in the space $\phi \in C_{0}^{1,1}\left(t \geq 0, r \geq r_{0}\right)$ so that initial and boundary values are accounted for in the weak formulation. (This is the simplest case in which to rigorously demonstrate the equivalence of several weak formulations of initial boundary value problems. More general domains can be handled in a similar manner.)

Note that because (2.25)-(2.27) involve only first derivatives of $A$ and $B$, and $A, B \in C^{0,1}$, it follows that (2.25)-(2.27) can be taken in the strong sense, that is, derivatives can be taken in the pointwise a.e. sense. The continuity of $A$ and $B$ imply also that the initial and boundary values are taken on strongly in any $C^{0,1}$ weak solution of $(2.25)-(2.27)$. On the other hand, 
equation (2.28) involves second derivatives, and so this last equation is the only one that requires a weak formulation. The weak formulation of (2.28) is thus obtained on domain $t \geq 0, r \geq r_{0} \geq 0$ by multiplying through by a test function $\phi \in C_{0}^{1,1}\left(r \geq r_{0}, t \geq 0\right)$ and integrating by parts. This yields the following weak formulation of (2.28):

$$
\begin{aligned}
0= & \int_{r_{0}}^{\infty} \int_{0}^{\infty}\left\{-\frac{B_{t} \phi_{t}}{r A B^{2}}-\frac{B_{t} \phi}{r}\left(-\frac{A_{t}}{A^{2} B^{2}}-\frac{2 B_{t}}{A B^{3}}\right)+\frac{A^{\prime} \phi^{\prime}}{r A B^{2}}\right. \\
& \left.+A^{\prime} \phi\left(-\frac{1}{r^{2} A B^{2}}-\frac{A^{\prime}}{r A^{2} B^{2}}-\frac{2 B^{\prime}}{r A B^{3}}\right)+\frac{\phi}{r A B^{2}} \Phi+\frac{2 \kappa r}{B} \phi T^{22}\right\} d r d t \\
& -\int_{r_{0}}^{\infty} \frac{B_{t}(r, 0) \phi(r, 0)}{r A(r, 0) B^{2}(r, 0)} d r+\int_{0}^{\infty} \frac{A^{\prime}\left(r_{0}, t\right) \phi\left(r_{0}, t\right)}{r_{0} A\left(r_{0}, t\right) B^{2}\left(r_{0}, t\right)} d t
\end{aligned}
$$

Our first proposition states that the weak formulation (6.4) of equation (2.28) may be replaced by the weak formulation of the conservation laws $\operatorname{div} T=0$, so long as $A$ and $B$ are in $C^{0,1}$ and $T^{i j} \in L^{\infty}$.

Proposition 4 Assume that $A, B \in C^{0,1}\left(r \geq r_{0}, t \geq 0\right), T^{i j} \in L^{\infty}(r \geq$ $r_{0}, t \geq 0$ ) and assume that $A, B$ and $T$ solve (2.25)-(2.27) strongly. Then $A$, $B$ and $T$ solve $T_{; i}^{1 i}=0$, (the 1-component of DivT $=0$ ), weakly if and only if $A, B$ and $T$ satisfy (6.4).

Proof: The proof strategy is to modify (6.4) and the weak form of conservation using (2.25)-(2.27) as identities, and then observe that the two are identical at an intermediate stage. To begin, substitute for $B_{t}$ and $A^{\prime}$ in several places in (6.4) to obtain the equivalent condition 


$$
\begin{aligned}
0= & \int_{r_{0}}^{\infty} \int_{0}^{\infty}\left\{\kappa T^{01} \varphi_{t}+\kappa T^{11} \varphi^{\prime}+\frac{\partial}{\partial r}\left(\varphi \frac{(B-1)}{r^{2} B^{2}}\right)+\varphi\left[-\frac{\partial}{\partial r}\left(\frac{B-1}{r^{2} B^{2}}\right)\right.\right. \\
& +\frac{B_{t}}{r}\left(\frac{A_{t}}{A^{2} B^{2}}+\frac{2 B_{t}}{A B^{3}}\right)+A^{\prime}\left(-\frac{1}{r^{2} A B^{2}}-\frac{A^{\prime}}{r A^{2} B^{2}}-\frac{2 B^{\prime}}{r A B^{3}}\right) \\
& \left.\left.+\frac{1}{r A B^{2}} \Phi+\frac{2 \kappa r}{B} T^{22}\right]\right\} d r d t \\
& +\kappa \int_{r_{0}}^{\infty} T^{01}(r, 0) \varphi(r, 0) d r+\kappa \int_{0}^{\infty} \varphi\left(r_{0}, t\right)\left[T^{11}\left(r_{0}, t\right) \frac{B\left(r_{0}, t\right)-1}{r_{0}^{2} B^{2}\left(r_{0}, t\right)}\right] d t \\
& =\int_{r_{0}}^{\infty} \int_{0}^{\infty}\left\{\kappa T^{01} \varphi_{t}+\kappa T^{11} \varphi^{\prime}+\varphi\left[\frac{B^{\prime}(B-2)}{r^{2} B^{3}}+2 \frac{(B-1)}{r^{3} B^{2}}\right.\right. \\
& +\frac{B_{t}}{r}\left(\frac{A_{t}}{A^{2} B^{2}}+\frac{2 B_{t}}{A B^{3}}\right)+A^{\prime}\left(-\frac{1}{r^{2} A B^{2}}-\frac{A^{\prime}}{r A^{2} B^{2}}-\frac{2 B^{\prime}}{r A B^{3}}\right) \\
& \left.\left.+\frac{1}{r A B^{2}} \Phi+\frac{2 \kappa r}{B} T^{22}\right]\right\} d r d t \\
& +\kappa \int_{r_{0}}^{\infty} T^{01}(r, 0) \varphi(x, 0) d r+\kappa \int_{0}^{\infty} \varphi\left(r_{0}, t\right) T^{11}\left(r_{0}, t\right) d t .
\end{aligned}
$$

Now, the weak form of conservation of energy-momentum is given by

$$
\begin{aligned}
0= & \int_{r_{0}}^{\infty} \int_{0}^{\infty}\left\{T^{01} \varphi_{t}+T^{11} \varphi^{\prime}-\left(\Gamma_{i 0}^{i} T^{01}+\Gamma_{i 1}^{i} T^{11}\right.\right. \\
& \left.\left.+\Gamma_{00}^{1} T^{00}+2 \Gamma_{01}^{1} T^{01}+\Gamma_{11}^{1} T^{11}+2 \Gamma_{22}^{1} T^{22}\right) \varphi\right\} d r d t \\
& +\int_{r_{0}}^{\infty} T^{01}(r, 0) \varphi(x, 0) d r+\int_{0}^{\infty} \varphi\left(r_{0}, t\right) T^{11}\left(r_{0}, t\right) d t
\end{aligned}
$$

Here, we have used the fact that $T^{22}=\sin ^{2} \theta T^{33}, T^{i j}=0$ if $i \neq j=2$ or 3 , and $\Gamma_{33}^{1}=\sin ^{2} \theta \Gamma_{22}^{1}$. Next, we calculate the connection coefficients $\Gamma_{j k}^{i}$ using (2.10) to obtain, 


$$
\begin{array}{ll}
\Gamma_{i 0}^{i}=\frac{1}{2}\left(\frac{A_{t}}{A}+\frac{B_{t}}{B}\right) & \Gamma_{i 1}^{i}=\frac{1}{2}\left(\frac{A^{\prime}}{A}+\frac{B^{\prime}}{B}+\frac{4}{r}\right) \\
\Gamma_{00}^{0}=\frac{A_{t}}{2 A} & \Gamma_{01}^{0}=\frac{A^{\prime}}{2 A} \\
\Gamma_{11}^{0}=\frac{B_{t}}{2 A} & \Gamma_{22}^{0}=0=\Gamma_{33}^{0} \\
\Gamma_{00}^{1}=\frac{A^{\prime}}{2 B} & \Gamma_{01}^{1}=\frac{B_{t}}{2 B} \\
\Gamma_{11}^{1}=\frac{B^{\prime}}{2 B} & \Gamma_{22}^{1}=-\frac{r}{B} \\
\Gamma_{33}^{1}=-\frac{r \sin ^{2} \theta}{B} . &
\end{array}
$$

Substituting the above formulas for $\Gamma_{j k}^{i}$ into (6.6) and using (2.25)-(2.27) as identities to eliminate some of the $T^{i j}$ in favor of expressions involving $A, B$ and $r$, we see that (6.6) is equivalent to:

$$
\begin{aligned}
0=\int_{r_{0}}^{\infty} \int_{0}^{\infty}\left\{T^{01} \varphi_{t}\right. & +T^{11} \varphi^{\prime}+\frac{\varphi}{\kappa}\left[\frac{1}{2}\left(\frac{A_{t}}{A}+\frac{3 B_{t}}{B}\right) \frac{B_{t}}{r A B^{2}}\right. \\
-\frac{1}{2}\left(\frac{A^{\prime}}{A}+\right. & \left.\frac{2 B^{\prime}}{B}+\frac{4}{r}\right) \frac{1}{r^{2} B^{2}}\left(r \frac{A^{\prime}}{A}-(B-1)\right) \\
& \left.\left.-\frac{A^{\prime}}{2 r^{2} A B}\left(r \frac{B^{\prime}}{B}+(B-1)\right)+2 \kappa \frac{r}{B} T^{22}\right]\right\} d r d t \\
& +\int_{r_{0}}^{\infty} T^{01}(r, 0) \varphi(r, 0) d r+\int_{0}^{\infty} \varphi\left(r_{0}, t\right) T^{11}\left(r_{0}, t\right) d t
\end{aligned}
$$

After some simplification, it is clear that (6.5) is equal to (6.8). This completes the proof of Proposition 4. 
We next show that the Einstein equations (2.25)-(2.27) together with $\operatorname{DivT}=0$ are overdetermined. Indeed, we show that for weak solutions with Lipschitz continuous metric, either (2.25) or (2.26) may be dropped in the sense that the dropped equation will reduce to an identity on any solution of the remaining equations, so long as the dropped equation is satisfied by either the initial or boundary data, as appropriate. The following proposition addresses the first case, namely, for weak solutions in which the metric is Lipschitz continuous, the first Einstein equation (2.25) reduces to an identity on solutions of (2.26)-(2.27), so long as (2.25) is satisfied by the intial data.

Theorem 15 Assume that $A, B \in C^{0,1}$ and $T \in L^{\infty}$ solve (2.26), (2.27) strongly, and solve DivT $=0$ weakly. Then if $A, B$, and $T$ satisfy (2.25) at $t=0$, then $A, B$, and $T$ also solve (2.25) for all $t>0$.

Proof: We first give the proof for the case when $A, B$ and $T$ are assumed to be classical smooth solutions of $(2.26),(2.27)$ and $\operatorname{Div} T=0$. This is followed by several lemmas necessary for the extension of this to the weak formulation, which is given in the final proposition. So to start, assume that $A, B$, and $T$ are all smooth functions, and thus solve DivT $=0$ strongly. For the proof in this case, define

$$
H^{i j} \equiv G^{i j}-\kappa T^{i j}
$$

Because (2.26) and (2.27) hold, $H^{01} \equiv H^{11} \equiv 0$. Since by assumption $T_{; i}^{i j}=0$ and since $G_{; i}^{i j}=0$ for any metric tensor as a consequence of the Bianchi identities, it follows that

$$
0=H_{; i}^{i j}=H_{, i}^{i j}+\Gamma_{i k}^{i} H^{k j}+\Gamma_{i k}^{j} H^{i k} .
$$

In particular, setting $j=0$,

$$
0=H_{; i}^{i 0}=H_{, i}^{i 0}+\Gamma_{i k}^{i} H^{k 0}+\Gamma_{i k}^{0} H^{i k} .
$$

By hypothesis, $H^{i 0}=0$ when $i \neq 0$. In addition, the connection coefficients $\Gamma_{i k}^{0}$ are zero unless $i$ or $k$ equal 0 or 1 . Therefore, (6.11) reduces to the linear ODE 


$$
0=H_{, 0}^{00}+\left(\Gamma_{i 0}^{i}+\Gamma_{00}^{0}\right) H^{00},
$$

at each fixed $r$. By hypothesis, $H^{00}$ is initially zero, and since we assume that $H^{00}$ is a smooth solution of $(6.12)$, it follows that $H^{00}$ must continue to be zero for all $t>0$.

Next, assume only that $A, B \in C^{0,1}$ and $T \in L^{\infty}$ so that (2.26), (2.27) hold strongly, (that is, in a pointwise a.e. sense), but that $\operatorname{DivT}=0$ is only known to hold weakly. In this case, the argument above has a problem because when $g \in C^{0,1}$, the Einstein tensor $G$, viewed as a second order operator on the metric components $A$ and $B$, can only be defined weakly when $A$ and $B$ are only Lipschitz continuous. It follows that the Bianchi identities, and hence the identity $\operatorname{Div} G=0$, (which involves first order derivatives of the components of the curvature tensor), need no longer be valid even in a weak sense. Indeed, $G$ can have delta function sources at an interface at which the metric is only Lipschitz continuous, c.f. [29]. However, the above argument only involves the 0'th component of DivG $=0$, and the 0'th component of $\operatorname{Div} G=0$ involves only derivatives of the components $G^{i 0}$, and as observed in (2.25), (2.26), these components only involve first derivatives of $A$ and $B$. Specifically, the weak formulation of $G_{; i}^{0 i}=0$ is given by,

$$
\begin{aligned}
& 0=\int_{r_{0}}^{\infty} \int_{0}^{\infty}\left\{-\phi_{i} G^{i 0}+\phi\left(\Gamma_{i k}^{i} G^{k 0}+\Gamma_{i k}^{0} G^{i k}\right)\right\} d r d t \\
& -\int_{r_{0}}^{\infty} \phi(r, 0) G^{00}(r, 0) d r-\int_{0}^{\infty} \phi\left(r_{0}, t\right) G^{10}\left(r_{0}, t\right) d t
\end{aligned}
$$

and since, by (2.25), (2.26), $G^{i 0}$ involves only first order derivatives of $A$ and $B$, it follows that the integrand in (6.13) is a classical function defined pointwise a.e. when $A, B \in C^{0,1}$. But (6.13) is identically zero for all smooth $A$ and $B$ because $\operatorname{Div} G=0$ is an identity. Thus, when $A, B \in C^{0,1}$, we can take a sequence of smooth functions $A_{\epsilon}, B_{\epsilon}$ that converge to $A$ and $B$ in the limit $\epsilon \rightarrow 0$, (c.f. Theorem 16 below), such that the derivatives converge a.e. to the derivatives of $A$ and $B$. It follows that we can take the limit $\epsilon \rightarrow 0$ (6.13) and conclude that (6.13) continues to hold under this limit. Putting this together with the fact that DivT $=0$ is assumed to hold weakly, we conclude that 


$$
H_{; i}^{0 i}=\left(G^{0 i}-T^{0 i}\right)_{; i}=0
$$

in the weak sense, which means that $H^{00}$ is in $L^{\infty}$ and satisfies the condition

$$
\begin{gathered}
0=\int_{r_{0}}^{\infty} \int_{0}^{\infty}\left\{-\phi_{0} H^{00}+\phi\left(\Gamma_{i 0}^{i}+\Gamma_{00}^{0}\right) H^{00}\right\} d r d t \\
-\int_{r_{0}}^{\infty} \phi(r, 0) G^{00}(r, 0) d r-\int_{r_{0}}^{\infty} \phi(r, 0) H^{00}\left(r_{0}, t\right) d r .
\end{gathered}
$$

Therefore, to complete the proof of Theorem 15, we need only show that if $A, B$ and $T$ solve (2.26), (2.27) classically and DivT $=0$ weakly, then a weak $L^{\infty}$ solution $H^{00}$, (i.e., that satisfies (6.14)), of (6.12) must be zero almost everywhere if it is zero initially. Thus it suffices to prove the following proposition:

Proposition 5 Assume that $H, f \in L_{l o c}^{\infty}(\mathbf{R} \times \mathbf{R})$. Then every $L_{l o c}^{\infty}$ weak solution to the initial value problem

$$
\begin{gathered}
H_{t}+f H=0 \\
H(x, 0)=H_{0}(x) .
\end{gathered}
$$

with initial data $H_{0} \equiv 0$ is unique, and identically equal to zero a.e., for all $t>0$.

Proof: We use the following standard theorem, [6],

Theorem 16 Let $U$ be any open subset of $\mathbf{R}^{n}$. Then $u \in W_{l o c}^{1, \infty}(U)$ if and only if $u$ is locally Lipschitz continuous in $U$, in which case the weak derivative of $u$ agrees with the classical pointwise a.e derivative as a function in $L_{l o c}^{\infty}(U)$.

Corollary $\mathbf{7}$ Let $u$ and $f$ be real valued functions, $u, f: \mathbf{R} \rightarrow \mathbf{R}$, such that $u, f \in L^{\infty}[0, T]$, and $u$ is a weak solution of the initial value problem 


$$
\begin{gathered}
u_{t}+f u=0, \\
u(0)=0,
\end{gathered}
$$

on the interval $[0, T]$. Then $u(t)=0$ for all $t \in[0, T]$.

Proof of Corollary: Statement (6.16) says that the distributional derivative $u_{t}$ agrees with the $L^{\infty}$ function $f u$ on the interval $[0, T]$, and thus we know that $u \in W_{l o c}^{1, \infty}(0, t)$. Therefore, by Theorem 16, $u$ is locally Lipschitz continuous on $(0, T)$, and the weak derivative $u_{t}$ agrees with the pointwise a.e. derivative of $u$ on $(0, T)$. Thus it follows from (6.16) that on any sub-interval $[a, b]$ of $[0, T]$ on which $u \neq 0$, we must have

$$
\frac{d}{d t}[\ln u]=\frac{u_{t}}{u}=-f, \text { a.e. }
$$

Moreover, since $u$ is Lipschitz continuous, both $u$ and $\ln (u)$ are absolutely continuous on $[a, b]$, so we can integrate (6.17) to see that

$$
u(t)=u(a) e^{-\int_{0}^{t} f(\xi) d \xi}
$$

for all $t \in[a, b]$. But $u$ is continuous, so (6.18) applies in the limit that $a$ decreases to the first value of $t=t_{0}$ at which $u\left(t_{0}\right)=0$. Thus (6.18) implies that $u(t)=0$ throughout $[a, b]$, and hence we must have $u(t)=0$ for all $t \in[0, T]$, and the corollary is proved.

The proof of Proposition 5 now follows because it is easy to show that if $H$ is an $L^{\infty}$ weak solution of $(6.15)$, then $H(x, \cdot)$ is a weak solution of the scalar ODE $H_{t}+f H=0$ for almost every $x$. (Just factor the test functions into products of the form $\phi_{1}(t) \phi_{2}(x)$.) 
Using Proposition 5, we see that if equation (2.25) holds on the initial data for a solution of (2.26), (2.27), and DivT $=0$, then equation (2.25) will hold for all $t$. By a similar argument, it follows that if (2.26) holds for the boundary data of a solution to (2.25), (2.27), and DivT $=0$, then (2.26) will hold for all $r$ and $t$. We record this in the following theorem:

Theorem 17 Assume that $A, B \in C^{0,1}$ and $T \in L^{\infty}$ solve (2.25), (2.27) strongly, and solve DivT $=0$ weakly, in $r \geq r_{0}, t \geq 0$. Then if $A, B$, and $T$ satisfy (2.26) at $r=r_{0}$, then $A, B$, and $T$ also solve (2.25) for all $r>r_{0}$.

\subsection{The Spherically Symmetric Einstein Equations For- mulated as a System of Hyperbolic Conservation Laws with Sources}

Conservation of energy and momentum is expressed by the equations

$$
\begin{aligned}
0 & =(\operatorname{DivT})^{j}=T_{; i}^{i j} \\
& =T_{, i}^{i j}+\Gamma_{i k}^{i} T^{k j}+\Gamma_{i k}^{j} T^{i k},
\end{aligned}
$$

which, in the case of spherical symmetry, can be written as the system of two equations:

$$
\begin{aligned}
& 0=T_{, 0}^{00}+T_{, 1}^{01}+\Gamma_{i k}^{i} T^{k 0}+\Gamma_{i k}^{0} T^{i k} \\
& 0=T_{, 0}^{01}+T_{, 1}^{11}+\Gamma_{i k}^{i} T^{k 1}+\Gamma_{i k}^{1} T^{i k}
\end{aligned}
$$

Substituting the expressions (6.7) for the connection coefficients (2.10) into (6.19) and (6.20), gives the equivalent system 


$$
\begin{aligned}
0= & T_{, 0}^{00}+T_{, 1}^{01}+\frac{1}{2}\left(\frac{2 A_{t}}{A}+\frac{B_{t}}{B}\right) T^{00}+\frac{1}{2}\left(\frac{3 A^{\prime}}{A}+\frac{B^{\prime}}{B}+\frac{4}{r}\right) T^{01} \\
& +\frac{B_{t}}{2 A} T^{11} \\
0= & T_{, 0}^{01}+T_{, 1}^{11}+\frac{1}{2}\left(\frac{A_{t}}{A}+\frac{3 B_{t}}{B}\right) T^{01}+\frac{1}{2}\left(\frac{A^{\prime}}{A}+\frac{2 B^{\prime}}{B}+\frac{4}{r}\right) T^{11}(6.2 \\
& +\frac{A^{\prime}}{2 B} T^{00}-2 \frac{r}{B} T^{22}
\end{aligned}
$$

Now if one could use equations to eliminate the derivative terms $A_{t}, A^{\prime}, B_{t}$ and $B^{\prime}$ in (6.21) and (6.22) in favor of of expressions involving the undifferentiated unknowns $A, B$ and $T$, then system (6.21), (6.22) would take the form of a system of conservation laws with source terms. Indeed, $T^{00}$ and $T^{01}$ serve as the conserved quantities, $T^{10}$ and $T^{11}$ are the fluxes, and what is left, written as a function of the undifferentiated variables $\left(A, B, T^{00}, T^{01}\right)$, would play the role of a source term. (For example, in a fractional step scheme designed to simulate the initial value problem, the variables $A$ and $B$ could be "updated" to time $t_{j}+\Delta t$ by the supplemental equations (2.25) and (2.27) or (2.26) and (2.27) after the conservation law step is implemented using the known values of $A$ and $B$ at time $t_{j}$. The authors will carry this out in detail in a subsequent paper.) The system then closes once one writes $T^{11}$ as a function of $\left(A, B, T^{00}, T^{01}\right)$. There is a problem here, however. Equations (2.25)-(2.27) can be used to eliminate the terms $A_{r}, B_{t}$ and $B_{r}$, but (6.21) and (6.22) also contain terms involving $A_{t}$, a quantity that is not given in the initial data and is not directly evolved by equations (2.25)-(2.27). The way to resolve this is to incorporate the $A_{t}$ term into the conserved quantities. For general equations involving $A_{t}$, this is not possible. A natural change of $T$ variables that eliminates the $A_{t}$ terms from $(6.21),(6.22)$, is to write the equations in terms of the values that $T$ takes in flat Minkowski space. That is, define $T_{M}$ in terms of $T$, by 


$$
\begin{aligned}
T^{00} & =\frac{1}{A} T_{M}^{00}, \\
T^{01} & =\frac{1}{\sqrt{A B}} T_{M}^{01}, \\
T^{11} & =\frac{1}{B} T_{M}^{11},
\end{aligned}
$$

where the subscript denotes Minkowski, c.f. (2.32)-(2.34). It then follows that $T_{M}$ is given by

$$
\begin{aligned}
T_{M}^{00} & =\left\{\left(p+\rho c^{2}\right) \frac{c^{2}}{c^{2}-v^{2}}-p\right\}, \\
T_{M}^{01} & =\left(p+\rho c^{2}\right) \frac{c v}{c^{2}-v^{2}}, \\
T_{M}^{11} & =\left\{\left(p+\rho c^{2}\right) \frac{v^{2}}{c^{2}-v^{2}}+p\right\},
\end{aligned}
$$

where $v$ denotes the fluid speed as measured by an inertial observer fixed with respect to the radial coordinate $r$, c.f. (2.35)-(2.37). (We discuss (6.24) in more detail in the last section.) Substituting (6.23) into (6.21), (6.22), the $A_{t}$ terms cancel out, and we obtain the system

$$
\begin{aligned}
0= & \left\{T_{M}^{00}\right\}_{, 0}+\left\{\sqrt{\frac{A}{B}} T_{M}^{01}\right\}_{, 1}+\frac{1}{2} \frac{B_{t}}{B}\left(T_{M}^{00}+T_{M}^{11}\right) \\
& +\frac{1}{2} \sqrt{\frac{A}{B}}\left(\frac{A^{\prime}}{A}+\frac{B^{\prime}}{B}+\frac{4}{r}\right) T_{M}^{01} \\
0= & \left\{T_{M}^{01}\right\}_{, 0}+\left\{\sqrt{\frac{A}{B}} T_{M}^{11}\right\}_{, 1}+\frac{1}{2} \sqrt{\frac{A}{B}}\left\{2 \frac{B_{t}}{\sqrt{A B}} T_{M}^{01}+\left(\frac{B^{\prime}}{B}+\frac{4}{r}\right) T_{M}^{11}\right. \\
& \left.+\frac{A^{\prime}}{A} T_{M}^{00}-4 r T^{22}\right\} .
\end{aligned}
$$

The following proposition states that system (6.25), (6.26) is equivalent, (in the weak sense), to the original system $\operatorname{DivT}=0$. 
Proposition 6 If $A$ and $B$ are given Lipschitz continuous functions defined on the domain $r \geq r_{0}, t \geq 0$, then $T_{M}$ is a weak solution of (6.25) and (6.26) if and only if $T$ is a weak solution of DivT $=0$ in this domain.

Proof: For simplicity, and without loss of generality, take the weak formulation with test functions compactly supported in $r>r_{0}, t>0$, so that the boundary integrals do not appear in the weak formulations. (Managing the boundary integrals is straightforward.) The variables $T_{M}^{i j}$ solve (6.25) weakly if

$$
\begin{aligned}
& 0=\int_{r_{0}}^{\infty} \int_{0}^{\infty}\left\{-T^{00} \varphi_{t}-\sqrt{\frac{A}{B}} T^{01} \varphi_{r}\right. \\
& \left.+\left[\frac{1}{2} \frac{B_{t}}{B}\left(T^{00}+T^{11}\right)+\frac{1}{2} \sqrt{\frac{A}{B}}\left(\frac{A^{\prime}}{A}+\frac{B^{\prime}}{B}+\frac{4}{r}\right) T^{01}\right] \varphi\right\} d r d t \\
& =\int_{r_{0}}^{\infty} \int_{0}^{\infty}\left\{-T_{M}^{00} A \varphi_{t}-T_{M}^{01} A \varphi_{r}\right. \\
& \left.+\left[\frac{1}{2} \frac{B_{t}}{B}\left(A T_{M}^{00}+B T_{M}^{11}\right)+\frac{1}{2} A\left(\frac{A^{\prime}}{A}+\frac{B^{\prime}}{B}+\frac{4}{r}\right) T_{M}^{01}\right] \varphi\right\} d r d t .
\end{aligned}
$$

Set $\psi=A \varphi$, whereby $A \varphi_{t}=\psi_{t}-\frac{A_{t}}{A} \psi$. Using this change of test function, (6.27) becomes 


$$
\begin{aligned}
0= & \int_{r_{0}}^{\infty} \int_{0}^{\infty}\left\{-T^{00} \psi_{t}+T^{00} \frac{A_{t}}{A} \psi-T^{01} \psi^{\prime}+T^{01} \frac{A^{\prime}}{A} \psi\right. \\
& \left.+\left[\frac{1}{2} \frac{B_{t}}{B}\left(T^{00}+\frac{B}{A} T^{11}\right)+\frac{1}{2}\left(\frac{A^{\prime}}{A}+\frac{B^{\prime}}{B}+\frac{4}{r}\right) T^{01}\right] \psi\right\} d r d t \\
= & \int_{r_{0}}^{\infty} \int_{0}^{\infty}\left\{-T^{00} \psi_{t}-T^{01} \psi^{\prime}+\left[\frac{1}{2}\left(\frac{2 A_{t}}{A}+\frac{B_{t}}{B}\right) T^{00}\right.\right. \\
& \left.\left.+\frac{1}{2}\left(\frac{3 A^{\prime}}{A}+\frac{B^{\prime}}{B}+\frac{4}{r}\right) T^{01}+\frac{B_{t}}{2 A} T^{11}\right] \psi\right\} d r d t
\end{aligned}
$$

which is the weak formulation of (6.21). We deduce that $T_{M}$ solves (6.25) for every Lipschitz continuous test function $\varphi$ if and only if $T$ solves (6.28), (the weak form of $T_{; i}^{0 i}=0$ ), for all Lipschitz continuous test functions $\psi$. That weak solutions of (6.26) are weak solutions of $T_{; i}^{1 i}=0$ follows by a similar argument.

It is now possible to use equations (2.25)-(2.27) as identities to substitute for derivatives of metric components $A$ and $B$, thereby eliminating the corresponding derivatives of $A$ and $B$ from the source terms of equations (6.25), (6.26). Doing this, we obtain the system of equations (2.46), (2.47), which was our goal. However, depending on the choice of equation to drop, either (2.25) or (2.26), it is not clear that if we use the dropped equation to substitute for derivatives in $(6.25),(6.26)$, that the resulting system of equations will imply that DivT $=0$ continues to hold, the assumption we based the substitution on in the first place. The following theorem states that (2.46), (2.47) is equivalent to DivT $=0$ in the weak sense: 
Theorem 18 Assume that $A, B$ are Lipschitz continuous functions, and that $T \in L^{\infty}$, on the domain $r \geq r_{0}, t \geq 0$. Assume also that (2.25) holds at $t=0$, and that (2.26) holds at $r=r_{0}$. Then $A, B, T$ are weak solutions of (2.25), (2.26), (2.27) and DivT $=0$ if and only if $A, B, T_{M}$ are weak solutions of either system (2.25), (2.27), (2.46), (2.47), or system (2.26), (2.27), (2.46), (2.47).

Proof: Without loss of generality, we consider the case when we drop equation (2.26), and use (2.25), (2.27) and DivT $=0$ to evolve the metric, and we ask whether we can take the modified system (2.46) and (2.47) in place of DivT $=0$. In this case, we must justify the use of (2.26) in eliminating the $B_{t}$ terms in going from DivT $=0$ to system (2.46) and (2.47). That is, it remains only to show that equations (2.25) and (2.27) together with system (2.46) and (2.47) imply that (2.26) holds, assuming (2.26) holds at $r=r_{0}$. (If so, then by substitution, it then follows that $\operatorname{DivT}=0$ also holds.) 
Note that we can almost reconstruct (6.21), the first component of DivT = 0 , by reverse substituting $(2.25),(2.27)$ into $(2.46)$. To see this, first note that we can add (2.25) and (2.27) to obtain

$$
\frac{A^{\prime}}{A}+\frac{B^{\prime}}{B}-r B \kappa\left(T_{M}^{00}+T_{M}^{11}\right)=0 .
$$

Equation (6.29) is an identity that we may add to (2.46) to obtain

$$
\begin{gathered}
0=\left\{T_{M}^{00}\right\}_{, 0}+\left\{\sqrt{\frac{A}{B}} T_{M}^{01}\right\}_{, 1}-\frac{1}{2} r \sqrt{A B} \kappa\left(T_{M}^{00}+T_{M}^{11}\right) T_{M}^{01} \\
+\frac{1}{2} \sqrt{\frac{A}{B}}\left(\frac{A^{\prime}}{A}+\frac{B^{\prime}}{B}+\frac{4}{r}\right) T_{M}^{01} .
\end{gathered}
$$

Adding and subtracting

$$
\frac{1}{2} \frac{B_{t}}{B}\left(T_{M}^{00}+T_{M}^{11}\right)
$$

to the RHS of (6.30) and using

$$
H^{01}=-\frac{B_{t}}{r B}-\sqrt{A B} \kappa T_{M}^{01},
$$

(c.f. (2.26) and (6.9)), we have

$$
\begin{aligned}
0=\left\{T_{M}^{00}\right\}_{, 0} & +\left\{\sqrt{\frac{A}{B}} T_{M}^{01}\right\}_{, 1}+\frac{1}{2} \sqrt{\frac{A}{B}}\left(\frac{A^{\prime}}{A}+\frac{B^{\prime}}{B}+\frac{4}{r}\right) T_{M}^{01} \\
& +\frac{1}{2} \frac{B_{t}}{B}\left(T_{M}^{00}+T_{M}^{11}\right)+\frac{1}{2} r\left(T_{M}^{00}+T_{M}^{11}\right) H^{01}
\end{aligned}
$$

Note that all but the last term on the RHS of (6.33) is equal to the first component of DivT, and so

$$
T_{; i}^{0 i}=-\frac{1}{2} r\left(T_{M}^{00}+T_{M}^{11}\right) H^{01}
$$

Therefore, if $A, B$, and $T_{M}$ are solutions to (2.25), (2.27), (6.33), and (6.22), it follows that 


$$
\begin{aligned}
H_{; i}^{i 0} & =G_{; i}^{i 0}-\kappa T_{; i}^{i 0} \\
& =\kappa \frac{r B^{2} T^{11}}{2} H^{01}
\end{aligned}
$$

because $G_{; i}^{i 0}=0$ is an identity. But $H^{00} \equiv 0$ holds because we assume (2.25), and hence (6.34) implies that

$$
H_{, 1}^{01}+f H^{01}=0
$$

where $f \equiv \Gamma_{i 1}^{i}+2 \Gamma_{01}^{1}-\kappa \frac{r B^{2} T^{11}}{2} \in L^{\infty}$. Since we assume that $H^{01}=0$ on the boundary $r=r_{0}$, it follows from Corollary 7 that $H^{01} \equiv 0$.

It remains to identify conditions under which $T_{M}^{11}$ is a function of $\left(T_{M}^{00}, T_{M}^{01}\right)$ assuming that $T$ has the form of a stress tensor for a perfect fluid, (6.24). A calculation shows that, in this case, the following simplifications occur:

$$
\begin{aligned}
T_{M}^{00}-T_{M}^{11} & =\rho c^{2}-p \\
T_{M}^{00} T_{M}^{11}-\left(T_{M}^{01}\right)^{2} & =p \rho c^{2} .
\end{aligned}
$$

Using (6.35) and (6.36) we see that only the first terms on the RHS of (2.46), (2.47) depend on $v$, and the only term that is not linear in $\rho$ and $p$ is the third term on the RHS of (2.47). We state and prove the following theorem:

Theorem 19 Assume that $0<p<\rho c^{2}, 0<\frac{d p}{d \rho}<c^{2}$. Then $T_{M}^{11}$ is a function of $T_{M}^{00}$ and $T_{M}^{01}$ so long as $(\rho, v)$ lie in the domain $D=\{(\rho, v): 0<\rho,|v|<c\}$.

Proof: We may write (6.35) and (6.36) in the form

$$
\begin{aligned}
T_{M}^{00}-T_{M}^{11} & =f_{1}(\rho), \\
T_{M}^{00} T_{M}^{11}-\left(T_{M}^{01}\right)^{2} & =f_{2}(\rho) .
\end{aligned}
$$

Since $\frac{d f_{1}}{d \rho}=c^{2}-p^{\prime} \geq c^{2}-\sigma^{2}>0$, it follows that the function $f_{1}$ is one-to-one with respect to $\rho$. Also, $\frac{d f_{2}}{d \rho}=p^{\prime} \rho c^{2}+p c^{2} \geq p c^{2}>0$, so the function $f_{2}$ is also one-to-one in $\rho$. Consequently, the function $h=f_{2} \cdot f_{1}^{-1}$ is one-to-one, and thus 


$$
T_{M}^{00} T_{M}^{11}-\left(T_{M}^{01}\right)^{2}=h\left(T_{M}^{00}-T_{M}^{11}\right)
$$

Now introduce the linear and invertible change of variables

$$
x=T_{M}^{00}-T_{M}^{11}, y=T_{M}^{01}, z=T_{M}^{11} \text {, whereby (6.39) becomes }
$$

$$
(x+z) z-y^{2}=h(x) .
$$

Equation (6.40) is quadratic in $z$, and so we may solve it directly, obtaining

$$
z=\frac{-x \pm \sqrt{x^{2}+4\left(y^{2}+h(x)\right)}}{2} .
$$

From (6.41), we conclude that for any $(x, y)$, there are two values of $z$, though only one of these will correspond to values of $\rho$ and $v$ in the domain $D$. That is, since

$$
x=T_{M}^{00}-T_{M}^{11}=\rho c^{2}-p>0,
$$

and $z=T_{M}^{11}>0$, it follows that there is at most one solution of (6.41) in the domain $D$, namely

$$
z=\frac{-x+\sqrt{x^{2}+4\left(y^{2}+h(x)\right)}}{2} .
$$

We conclude that if $(\rho, v)$ lies in the domain $D$, then for each value of $T_{M}^{00}$ and $T_{M}^{01}$, there exists precisely one value of $T_{M}^{11}$.

A calculation shows that in the case $p=\sigma^{2} \rho, \sigma=$ constant, the formula for $T_{M}^{11}$ in terms of $\left(T_{M}^{00}, T_{M}^{01}\right)$ is given by

$$
T_{M}^{11}=\frac{1+2 K_{*}}{2 K_{*}}\left\{T_{M}^{00}-\sqrt{\left(T_{M}^{00}\right)^{2}-\frac{4 K_{*}}{\left(1+2 K_{*}\right)^{2}}\left(K_{*}\left(T_{M}^{00}\right)^{2}+\left(T_{M}^{01}\right)^{2}\right)}\right\}
$$

where 


$$
K_{*}=\frac{\sigma^{2} c^{2}}{\left(c^{2}-\sigma^{2}\right)^{2}} \text {. }
$$

\subsection{Summary of the Weak Formulations}

Our results concerning the weak formulation of the Einstein equations (2.25)(2.28) assuming spherical symmetry given in Theorem 18 can be summarized as follows. Assume that $A, B$ are Lipschitz continuous functions, and that $T \in L^{\infty}$, on the domain $r \geq r_{0}, t \geq 0$. Then (2.25)-(2.28) are equivalent to two different systems which take the form of a system of conservation laws with source terms. In the first case, we have shown that weak solutions of the system (2.25), (2.27) together with equations (6.25), (6.26) (for DivT =0), will solve (2.25)-(2.28) weakly, so long as (2.26) holds at $r=r_{0}$. This reduces the Einstein equations with spherical symmetry to a system of equations of the general form

$$
\begin{gathered}
u_{t}+f(u, A, B)_{x}=\bar{g}\left(u, A, B, A^{\prime}, B_{t}, B^{\prime}, x\right), \\
A_{x}=h^{0}(u, A, B, x), \\
B_{x}=h^{1}(u, A, B, x),
\end{gathered}
$$

where $u=\left(T_{M}^{00}, T_{M}^{01}\right)$ agree with the conserved quantities that appear in the conservation law div $T_{M}=0$ in flat Minkowski space. (Here "prime" denotes $\frac{\partial}{\partial x}$ since we are using $x$ in place of $r$.) It is then valid to use equations (2.25)(2.27) to eliminate all derivatives of $A$ and $B$ from the RHS of system (6.46), by which we obtain the system $(2.25),(2.27),(2.46),(2.47)$, a system that closes to make a nonlinear system of conservation laws with source terms, taking the general form

$$
\begin{gathered}
u_{t}+f(u, A, B)_{x}=g(u, A, B, x), \\
A_{x}=h^{0}(u, A, B, x), \\
B_{x}=h^{1}(u, A, B, x),
\end{gathered}
$$

which reproduces $(2.48),(2.49)$ of Section 1. Weak solutions of (6.49) will satisfy (2.26) so long as (2.26) is satisfied on the boundary $r=r_{0}$. 
In the second case, we have shown that weak solutions of the system (2.26), (2.27) together with equations (6.25), (6.26) (for DivT =0), will solve (2.25)-(2.28) weakly, so long as $(2.25)$ holds at $t=0$. This reduces the Einstein equations with spherical symmetry to an alternative system of equations of the general form

$$
\begin{gathered}
u_{t}+f(u, A, B)_{x}=\bar{g}\left(u, A, B, A^{\prime}, B_{t}, B^{\prime}, x\right), \\
A_{x}=h^{0}(u, A, B, x), \\
B_{t}=h_{*}^{1}(u, A, B, x) .
\end{gathered}
$$

It is then valid to use equations (2.25)-(2.27) to eliminate all derivatives of $A$ and $B$ from the RHS of system (6.50), by which we obtain the system (2.26), (2.27), (2.46), (2.47), a system that closes to make a nonlinear system of conservation laws with source terms, taking the general form

$$
\begin{gathered}
u_{t}+f(u, A, B)_{x}=g(u, A, B, x), \\
A_{x}=h^{0}(u, A, B, x), \\
B_{t}=h_{*}^{1}(u, A, B, x) .
\end{gathered}
$$

Weak solutions of (6.53) will satisfy (2.25) so long as (2.25) is satisfied at $t=0$.

\subsection{Wave Speeds}

In this section we conclude by calculating the wave speeds associated with system (2.46)-(2.47).

The easiest way to calculate the wave speeds for the fluid is from the Rankine-Hugoniot jump conditions in the limit as the shock strength tends to zero. To start, note that the components of the 4-velocity $\mathbf{w}$ for a spherically symmetric fluid (2.18) are $w^{0}=\frac{d t}{d s}, w^{1}=\frac{d r}{d s}, w^{2}=w^{3}=0$. Since $-1=$ $g(\mathbf{w}, \mathbf{w})$, the components $w^{0}$ and $w^{1}$ are not independent, and in particular, $-1=-\left(w^{0}\right)^{2} A+\left(w^{1}\right)^{2} B$. We define fluid speed $v$ as the speed measured by an observer fixed in $(t, r)$ coordinates. That is, the speed is the change in distance per change in time as measured in an orthonormal frame with timelike vector parallel to $\partial_{t}$ and spacelike vector parallel to $\partial_{r}$. It follows that the speed is given by $v=x / a$, where 


$$
\mathbf{w}=a \frac{\partial_{t}}{\sqrt{-g_{00}}}+x \frac{\partial_{r}}{\sqrt{g_{11}}} .
$$

Taking the inner product of $\mathbf{w}$ with $\partial_{t}$ and then with $\partial_{r}$, we find that $a=$ $w^{0} \sqrt{-g_{00}}$ and $x=w^{1} \sqrt{g_{11}}$, and hence

$$
v=\frac{w^{1}}{w^{0}} \sqrt{\frac{B}{A}},
$$

whereby,

$$
\left(w^{0}\right)^{2}=\frac{1}{A\left(c^{2}-v^{2}\right)} .
$$

Using (6.57) and (6.58) in (2.18), it follows that the components of the energymomentum tensor take the following simplified form, which is valid globally in the $(t, r)$ coordinate system:

$$
\begin{aligned}
T^{00} & =\frac{1}{A}\left\{\left(p+\rho c^{2}\right) \frac{c^{2}}{c^{2}-v^{2}}-p\right\} \\
T^{01} & =\frac{1}{\sqrt{A B}}\left(p+\rho c^{2}\right) \frac{c v}{c^{2}-v^{2}} \\
T^{11} & =\frac{1}{B}\left\{\left(p+\rho c^{2}\right) \frac{v^{2}}{c^{2}-v^{2}}+p\right\} .
\end{aligned}
$$

Note that these components are equal to the components of the stress tensor in flat Minkowski space, times factors involving $A$ and $B$ that account for the fact that the spacetime is not flat. Using (2.32)-(2.34) we can write the Rankine-Hugoniot jump conditions in the form

$$
\begin{aligned}
& s\left[T_{M}^{00}\right]=\sqrt{\frac{A}{B}}\left[T_{M}^{01}\right], \\
& s\left[T_{M}^{01}\right]=\sqrt{\frac{A}{B}}\left[T_{M}^{11}\right] .
\end{aligned}
$$

From (6.59)-(6.60), we deduce that wave speeds for the system (2.46)-(2.47) are $\sqrt{A / B}$ times the wave speeds in the Minkowski metric case, and this holds globally throughout the $(t, r)$ coordinate system. (See [28].) Eliminating $s$ from (6.59) and (6.60), yields 


$$
\left[T_{M}^{01}\right]^{2}=\left[T_{M}^{00}\right]\left[T_{M}^{11}\right]
$$

Now take the left fluid state on a shock curve to be $\left(\rho_{L}, v_{L}\right)$, and the right fluid state to be $(\rho, v)$. For a spherically symmetric perfect fluid, (6.61) defines the right velocity $v$ as a function of the right density $\rho$. Then to obtain the fluid wave speeds, just substitute this function into (6.59), solve for $s$, and take the limit as $\rho \rightarrow \rho_{L}$. Following this procedure, (6.61) simplifies to

$$
\frac{\left(v-v_{L}\right)^{2}}{\left(c^{2}-v^{2}\right)\left(c^{2}-v_{L}^{2}\right)}=\frac{[p][\rho]}{\left(p+\rho c^{2}\right)\left(p_{L}+\rho_{L} c^{2}\right)} .
$$

Note that equation (6.62) can be written as a quadratic in $v$, and hence there are two solutions. The '+'solutions will yield the 2-shocks, and the '-' the 1-shocks. Dividing both sides of $(6.62)$ by $\left(\rho-\rho_{L}\right)^{2}$ and taking the limit as $\rho \rightarrow \rho_{L}$, we see that

$$
\frac{d p}{d \rho}=\frac{\left(p+c^{2} \rho\right)^{2}}{\left(c^{2}-v^{2}\right)^{2}}\left(\frac{d v}{d \rho}\right)^{2} .
$$

Solving (6.60) for $s$ we obtain,

$$
s=\sqrt{\frac{A}{B}} \frac{\left[\left(p+\rho c^{2}\right) \frac{v^{2}}{c^{2}-v^{2}}+p\right]}{\left[\left(p+\rho c^{2}\right) \frac{c v}{c^{2}-v^{2}}\right]},
$$

and taking the limit as $\rho \rightarrow \rho_{L}$, we obtain

$$
\begin{aligned}
\lambda_{ \pm} & =\sqrt{\frac{A}{B}} \frac{\left[\left(p^{\prime}+c^{2}\right) \frac{v^{2}}{c^{2}-v^{2}}+\left(p+\rho c^{2}\right) \frac{2 v v^{\prime}\left(c^{2}-v^{2}\right)+2 v^{3} v^{\prime}}{\left(c^{2}-v^{2}\right)^{2}}+p^{\prime}\right]}{\left[\left(p^{\prime}+c^{2}\right) \frac{c v}{c^{2}-v^{2}}+\left(p+\rho c^{2}\right) \frac{c v^{\prime}\left(c^{2}-v^{2}\right)+2 c v^{2} v^{\prime}}{\left(c^{2}-v^{2}\right)^{2}}\right]} \\
& =\sqrt{\frac{A}{B}} \frac{\left[\left(p^{\prime}+c^{2}\right) \frac{v^{2}}{c^{2}-v^{2}}+\left(p+\rho c^{2}\right) \frac{2 c^{2} v v^{\prime}}{\left(c^{2}-v^{2}\right)^{2}}+p^{\prime}\right]}{\left[\left(p^{\prime}+c^{2}\right) \frac{c v}{c^{2}-v^{2}}+\left(p+\rho c^{2}\right) \frac{c v^{\prime}\left(c^{2}+v^{2}\right)}{\left(c^{2}-v^{2}\right)^{2}}\right]} .
\end{aligned}
$$

(Here the plus/minus on RHS is determined by the two possible signs of $v^{\prime}=d v / d \rho$ as allowed by (6.63).) After substituting for $d v / d \rho$ using (6.63), and simplifying, we obtain 


$$
\begin{aligned}
\lambda_{ \pm} & =\sqrt{\frac{A}{B}} \frac{\left[\left(p^{\prime}+c^{2}\right) \frac{v^{2}}{c^{2}-v^{2}} \pm \frac{2 c^{2} v \sqrt{p^{\prime}}}{\left(c^{2}-v^{2}\right)}+p^{\prime}\right]}{\left[\left(p^{\prime}+c^{2}\right) \frac{c v}{c^{2}-v^{2}} \pm \frac{c\left(c^{2}+v^{2}\right) \sqrt{p^{\prime}}}{\left(c^{2}-v^{2}\right)}\right]}, \\
& =\sqrt{\frac{A}{B}} \frac{\left[\left(p^{\prime}+c^{2}\right) v^{2} \pm 2 c^{2} v \sqrt{p^{\prime}}+p^{\prime}\left(c^{2}-v^{2}\right)\right]}{\left[\left(p^{\prime}+c^{2}\right) c v \pm c\left(c^{2}+v^{2}\right) \sqrt{p^{\prime}}\right]} \\
& =c \sqrt{\frac{A}{B}} \frac{\left[v^{2} \pm 2 v \sqrt{p^{\prime}}+p^{\prime}\right]}{\left[v p^{\prime} \pm\left(c^{2}+v^{2}\right) \sqrt{p^{\prime}}+c^{2} v\right]}, \\
& =c \sqrt{\frac{A}{B}} \frac{\left[v \pm \sqrt{p^{\prime}}\right]^{2}}{\left[v \pm \sqrt{p^{\prime}}\right]\left[c^{2} \pm v \sqrt{p^{\prime}}\right]}
\end{aligned}
$$

This gives,

Proposition 7 The wave speeds of the general relativistic Euler equations (6.49) are

$$
\lambda_{ \pm}=c \sqrt{\frac{A}{B}} \frac{\sqrt{p^{\prime}} \pm v}{v \sqrt{p^{\prime}} \pm c^{2}} .
$$

The following proposition demonstrates that the system (2.46)-(2.47) is strictly hyperbolic whenever the particles are moving at less than the speed of light:

Proposition 8 Assume that

$$
|v|<c,
$$

so that the particle trajectory has a timelike tangent vector. Then wave speeds for the general relativistic Euler equations (2.46)-(2.47) satisfy $\lambda_{-}<\lambda_{+}$.

Proof: To determine where the wave speeds are equal, set $\lambda_{-}$equal to $\lambda_{+}$ and solve for $v$ to obtain $v^{2}=c^{2}$. Next, substitute $v=0$ into $\lambda_{-}$and $\lambda_{+}$to verify that $\lambda_{-}<\lambda_{+}$when $v^{2}<c^{2} A / B$. Proposition 8 follows directly. 
As a final comment, we note that Proposition 8 is true because it is true in a locally inertial coordinate system centered at any point $P$ in spacetime. Indeed, in such a coordinate system, the connection coefficients vanish at $P$, and the metric components match those of the Minkowski metric to first order in a neighborhood of $P$. As a result, the general relativistic Euler equations reduce to the classical relativistic Euler equations at $P$. Since it is known in Special Relativity that the Euler equations are strictly hyperbolic for timelike particles, [28], it follows that the same must be true in General Relativity. Other pointwise properties, such as genuine nonlinearity and the Lax entropy inequalities, $[27,15]$, can be verified for the spherically symmetric general relativistic equations in a similar manner. 
Because $A$ and $B$ enter as undifferentiated source terms, it follows from (2.46)-(2.47) that for spherically symmetric flow, the only wave speeds in the problem will be the characteristic speeds for the fluid. Loosely speaking, the gravitational field is "dragged along" passively by the fluid when spherical symmetry is imposed. From this we conclude that there is no lightlike propagation, (that is, no gravity waves), in spherical symmetry, even when there is matter present. For the empty space equations, this is the conclusion of Birkoff's theorem, [42].)

\section{References}

[1] A.M. Anile, Relativistic Fluids and Magneto-Fluids, Cambridge Monographs on Mathematical Physics, Cambridge University Press, 1989.

[2] D. Christodoulou, Private communication.

[3] R. Courant and K. Friedrichs, Supersonic Flow and Shock-Waves, Wiley-Interscience, 1948. 1972.

[4] B.A. Dubrovin, A.T. Fomenko and S.P.Novikov, Modern GeometryMethods and Applications, Springer Verlag, 1984.

[5] A. Einstein, Der feldgleichungen der gravitation, Preuss. Akad. Wiss., Berlin, Sitzber. (1915b), pp. 844-847.

[6] Lawrence C. Evans, Partial Differential Equations, Vol 3A, Berkeley Mathematics Lecture Notes, 1994.

[7] J. Glimm, Solutions in the large for nonlinear hyperbolic systems of equations, Comm. Pure Appl. Math., 18,(1965), pp. 697-715.

[8] J. Groah, Solutions of the Relativistic Euler equations in non-flat spacetimes, Doctoral Thesis, UC-Davis.

[9] J. Groah and B. Temple, A shock wave formulation of the Einstein equations, Methods and Applications of Analysis, 7, No. 4, (2000), pp. 793-812.

[10] J. Groah and B. Temple, Shock-wave solutions of the spherically symmetric Einstein equations with perfect fluid sources: existence and consistency for the initial value problem, (UC-Davis Preprint). 
[11] S.W. Hawking and G.F.R. Ellis, The Large Scale Structure of Spacetime, Cambridge University Press, 1973.

[12] W. Israel, Singular hypersurfaces and thin shells in General Relativity, IL Nuovo Cimento, XLIV B, No. 1, (1966), 1-14.

[13] M. Johnson and C. McKee, Relativistic hydrodynamics in one dimension, Phys. Rev. D, 3, no. 4, (1971), p. 858-863.

[14] P.D. Lax, Hyperbolic systems of conservation laws, II, Comm. Pure Appl. Math., 10,(1957), pp. 537-566.

[15] P.D. Lax, Shock-waves and entropy. In: Contributions to Nonlinear Functional Analysis, ed. by E. Zarantonello, Academic Press, 1971, pp. 603-634.

[16] T.P. Liu, Private communication.

[17] M. Luskin and B. Temple, The existence of a global weak solution of the waterhammer problem, Comm. Pure Appl. Math., 35, (1982), pp. 697-735.

[18] T. Makino, K. Mizohata and S. Ukai, Global weak solutions of the relativistic Euler equations with spherical symmetry, Japan J. Ind. and Appl. Math., 14, No. 1, (1997), pp. 125-157.

[19] D. Marchesin and P.J. Paes-Leme, A Riemann problem in gas dynamics with bifurcation, PUC Report MAT 02/84, 1984.

[20] G.C. McVittie, Gravitational collapse to a small volume, Astro. Phys. Jour., 140, (1964), pp. 401-416.

[21] C. Misner and D. Sharp, Relativistic equations for adiabatic, spherically symmetric gravitational collapse, Phys. Rev., 26, (1964), p. 571-576.

[22] C. Misner, K. Thorne, and J. Wheeler, Gravitation, Freeman, 1973.

[23] T. Nishida, Global solution for an initial boundary value problem of a quasilinear hyperbolic system, Proc. Jap. Acad., 44, (1968), pp. 642-646.

[24] T. Nishida and J. Smoller Solutions in the large for some nonlinear hyperbolic conservation laws, Comm. Pure Appl. Math., 26, (1973), pp. 183-200. 
[25] J.R. Oppenheimer and J.R. Snyder, On continued gravitational contraction, Phys. Rev., 56, (1939), pp. 455-459.

[26] J.R. Oppenheimer and G.M. Volkoff, On massive neutron cores, Phys. Rev., 55(1939), pp. 374-381.

[27] J. Smoller, Shock Waves and Reaction Diffusion Equations, Springer Verlag, 1983.

[28] J. Smoller and B. Temple Global solutions of the relativistic Euler equations, Comm. Math. Phys., 157, (1993), p. 67-99.

[29] J. Smoller and B. Temple, Shock-wave solutions of the Einstein equations: the Oppenheimer-Snyder model of gravitational collapse extended to the case of non-zero pressure, Arch. Rat. Mech. Anal., 128, (1994), pp 249-297.

[30] J. Smoller and B. Temple, Astrophysical shock-wave solutions of the Einstein equations, Phys. Rev. D, 51, No. 6, (1995), pp. 2733-2743.

[31] J. Smoller and B. Temple, General relativistic shock-waves that extend the Oppenheimer-Snyder model, Arch. Rat. Mech. Anal. 138, (1997), pp. 239-277.

[32] J. Smoller and B. Temple, Shock-wave solutions in closed form and the Oppenheimer-Snyder limit in General Relativity, with J. Smoller, SIAM J. Appl. Math, 58, No. 1, (1988), pp. 15-33.

[33] J. Smoller and B. Temple, On the Oppenheimer-Volkov equations in General Relativity, with J. Smoller, Arch. Rat. Mech. Anal., 142, (1998), 177-191.

[34] J. Smoller and B. Temple, Solutions of the Oppenheimer-Volkoff equations inside 9/8'ths of the Schwarzschild radius, with J. Smoller, Commun. Math. Phys. 184, (1997), 597-617.

[35] J. Smoller and B. Temple, Cosmology with a shock wave, Commun. Math. Phys., 210, (2000), 275-308. 
[36] J. Smoller and B. Temple, Shock-wave solutions of the Einstein equations: A general theory with examples, (to appear), Proceedings of European Union Research Network's 3rd Annual Summerschool, Lambrecht (Pfalz) Germany, May 16-22, 1999.

[37] A. Taub, Approximate Solutions of the Einstein equations for isentropic motions of plane-symmetric distributions of perfect fluids,, Phys. Rev., 107, no. 3, (1957), pp. 884-900.

[38] K. Thompson, The special relativistic shock tube, J. Fluid Mech., 171, (1986), pp. 365-375.

[39] R. Tolman, Relativity, Thermodynamics and Cosmology, Oxford University Press, 1934.

[40] R. Tolman, Static Solutions of Einstein's Field Equations for Spheres of Fluid, Physical Review, 55, (1939), pp. 364-374.

[41] R.M. Wald, General Relativity, University of Chicago Press, 1984.

[42] S. Weinberg, Gravitation and Cosmology: Principles and Applications of the General Theory of Relativity, John Wiley \& Sons, New York, 1972. 